Portland State University

PDXScholar

$1-1-1981$

\title{
Physical effects of 3-phenylindole on ion transport across bilayer lipid membranes
}

\author{
Barbara A. Sinha \\ Portland State University
}

Follow this and additional works at: https://pdxscholar.library.pdx.edu/open_access_etds

\section{Let us know how access to this document benefits you.}

\section{Recommended Citation}

Sinha, Barbara A., "Physical effects of 3-phenylindole on ion transport across bilayer lipid membranes" (1981). Dissertations and Theses. Paper 474.

https://doi.org/10.15760/etd.474

This Dissertation is brought to you for free and open access. It has been accepted for inclusion in Dissertations and Theses by an authorized administrator of PDXScholar. Please contact us if we can make this document more accessible: pdxscholar@pdx.edu. 
PHYSICAL EFFECTS OF 3-PHENYLINDOLE ON ION

TRANSPORT ACROSS BILAYER LIPID MEMBRANES

by

BARBARA A. SINHA

A dissertation submitted in partial fulfillment of the requirements for the degree of

Portland State University

1981 
TO THE OFFICE OF GRADUATE STUDIES AND RESEARCH:

The members of the Committee approve the dissertation of

Barbara A. Sinha presented July 31, 1981.

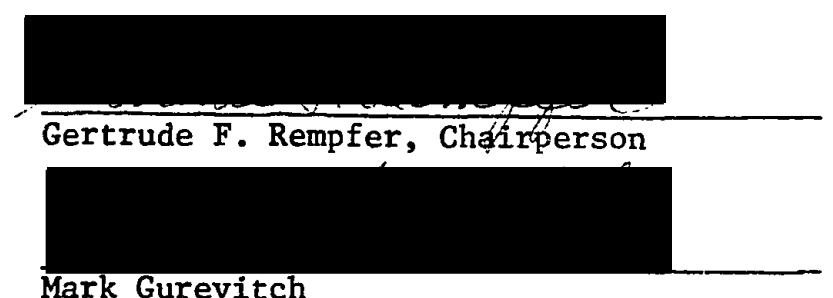

Mark Gurevitch
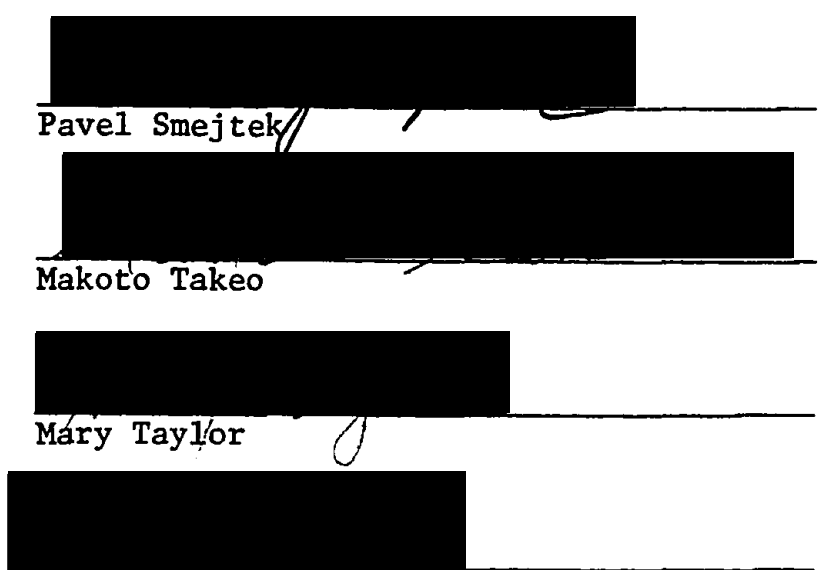

Horace F. White

APPROVED:

Mark Gurèvitch, Head, Department of Physics

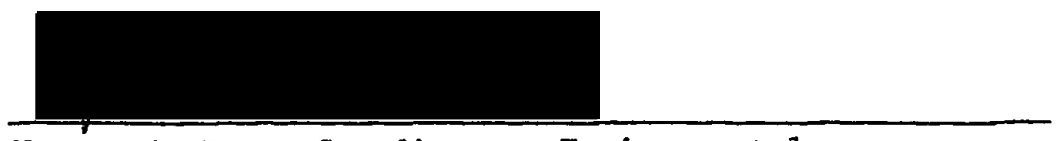

Norman C. Rose, Coordinator, Environmental

Sciences and Resources Ph.D. Program

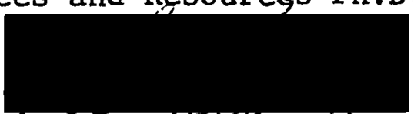

Stanley E. Rauch, Dean of Graduate Studies and Research 
AN ABSTRACT OF THE DISSERTATION OF Barbara A. Sinha for the Doctor of Philosophy in Environmental Sciences and Resources - Physics presented July 31, 1981.

Title: Physical Effects of 3-phenylindole on Ion Transport Across Bilayer Lipid Membranes APPROVED BY MEMBERS OF THE DISSERTATION COMMITTEE:

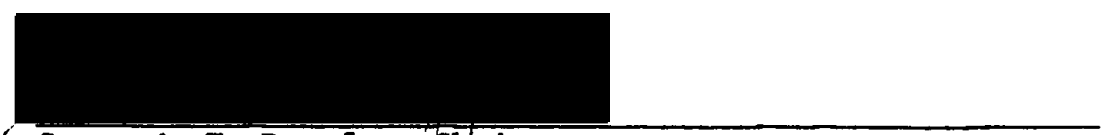

Gertrude F. Rempfer, Chairperson

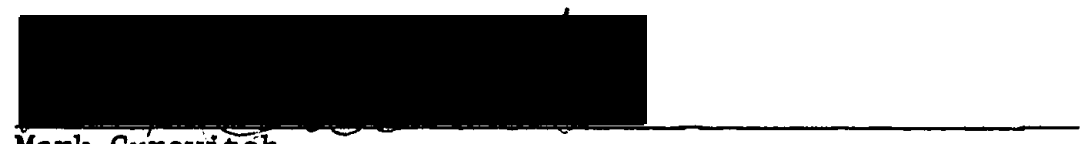
Mark Gurevitch

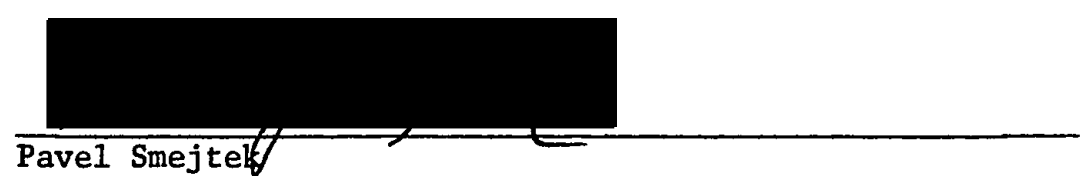
Pavel Smejtely

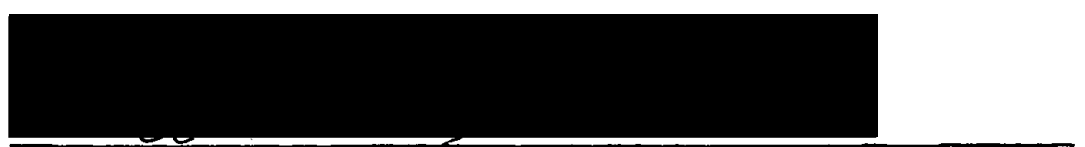

Makoto Takeo
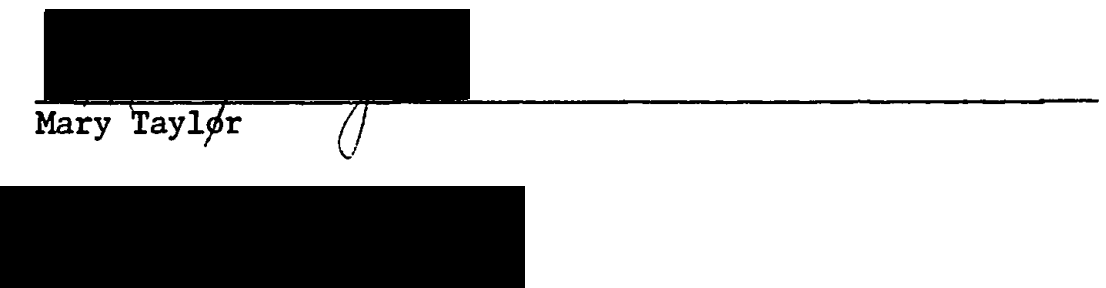

Horace F. White 


\begin{abstract}
The compound 3-phenylindole (3PI) is a particularly active antimicrobial which interacts with phospholipids in fungal mycelia membranes, and which strongly inhibits the uptake of phosphate into fungal mycelia. The physical effects of 3PI on ion transport across bilayer lipid membranes composed of phosphatidylcholine/cholesterol have been investigated using three lipophilic ions and one ion/carrier complex. It was found that 3PI increased the electrical conductivity induced by the lipophilic cation (tetraphenylarsonium) and by the positively charged complex (nonactin- $\mathrm{K}^{+}$) by several orders of magnitude whereas 3PI decreased the conductivity induced by the two lipophilic anions (tetraphenylborate, dipicrylamine) by a factor of less than ten. These conductivity changes are explained as a combination of changes in the electrostatic and in the non-electrostatic properties of the bilayer. The electrostatic potential of the bilayer interior was shown to decrease in the presence of 3PI, a phenomenon which was also confirmed by measurements of the surface potential of phosphatidylcholine/ cholesterol monolayers. The changes in non-electrostatic properties of the bilayer are qualitatively discussed in terms of increased bilayer fluidity or decreased bilayer thickness brought about by the presence of 3PI. The partition coefficient of the lipophilic anions, as determined by the voltage-step transient current technique, decreased slightly when 3PI was present in the aqueous phase.
\end{abstract}


From the voltage dependence of the normalized steady state conductivity it was shown that 3PI did not kinetically limit tetraphenylarsonium transport but that it did kinetically limit nonactin- $\mathrm{K}^{+}$ transport at concentrations greater than $25 \mu \mathrm{M} 3 \mathrm{PI}$. The theory of carrier-mediated transport predicts the occurrence of transient currents in the kinetically limited regime but no transients were detected for nonactin- $\mathrm{K}^{+}$in the presence of $80 \mu \mathrm{M} 3 \mathrm{PI}$.

A method for analyzing the adsorption of neutral lipophilic molecules onto lipid monolayers has been presented, and by this method it was found that the partition coefficient of 3PI onto phosphatidylcholine/cholesterol monolayers was $1.3 \times 10^{-4} \mathrm{~m}$ and that the maximum adsorbed surface number density of $3 P I$ was $1.1 \times 10^{-6} \mathrm{moles} / \mathrm{m}^{2}$. From the experimental changes in monolayer surface potential as a function of adsorbed surface number density, a value for the normal component of the dipole moment of 3PI was obtained. 


\section{ACKNOWLEDGEMENTS}

This dissertation is dedicated to the one who inspired me on countless occasions, who developed my skepticism, who was my toughest critic and my closest friend, Dipen Sinha. Dr. Gertrude Rempfer has been constantly optimistic about my research over the years. Her keen insight into physical matters is much appreciated. Part of what I gained in graduate school was confidence in my ability to teach and interact with students; I am indebted to Mrs. Dressler and Dr. Pickar for the opportunities they placed in my path. I would like to thank the members of my Supervisory Committee for the ir lively participation at our meetings. I am grateful to $\mathrm{Dr}$. Takeo for his meticulous reading of my dissertation and to Dr. Mary Taylor for patiently expounding on microbiology. I am also grateful to Mrs. Celeste Rehor for helping to keep me balanced during my last year at Portland State University.

Scientific experiments are often as much an art as a science and I would like to thank Mali Paulis-Illangasekare and Ranjith Jayaweera for giving me advice based on their experience and Julian Hobbs for taking time to introduce me to the computer. I am indebted to $\mathrm{Dr}$. Lloyd Dolby for the synthesis of 3-phenylindole. The smooth working of the monolayer set-up would not have been possible without the contributions of Mr. Janacek, Mr. Zupan, and Mr. Arakelian. I am obliged to $\mathrm{Mr}$. Thannum for the design and construction of the computer interfacing. Mr. Boileau and Mr. McLaughl in were always ready to help me in obtaining and repairing equipment. 
I am grateful to Dr. Seaman, the people in Dr. Seaman's group, and $\mathrm{Dr}$. Hsu, who all showed me by example how to design and document a project. I wish to thank Dr. Tuljapurkar for his concern for the scientific part of my soul and his efforts to expand that part. And finally, I want to thank Dr. Smejtek, esteemed leader of the membrane biophysics group, for bel jeving in my intellectual and experimental capabilities. I hope that his earnest pursuit of scientific knowledge and his enthusiasm for 1 ife are abundantly rewarded. 
TABLE OF CONTENTS

PAGE

ACKNOWLEDGMENTS ...............................

LIST OF TABLES .................................. viii

LIST OF FIGURES .................... . ix

CHAPTER

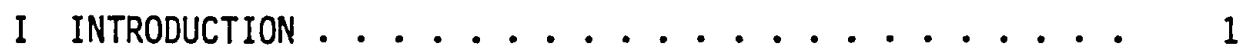

Structure and Function of Biological Membranes . . 1

Ion Transport in Biological Membranes and Lipid Bilayers ........... 10

Disruption of Membrane Structure and Function by Pesticides .......... . 17

Effect of 3-phenylindole on Fungal Cell

Membrane Structure and Function ........ 21

Project Description . . . . . . . . . 25

II THE EFFECT OF 3-PHENYLINDOLE ON LIPID MONOLAYERS . . . . 29

Adsorption to Lipid Monolayers . . . . . . . 29

Electrostatic Potential Changes due to Adsorbed 3-phenylindole ........ 31

Materials...................... 32

Procedure ................. 34

Results.......................... 36

Discussion ..................... 44 
II ENHANCEMENT OF TETRAPHENYLARSONIUM

TRANSPORT BY 3-PHENYLINDOLE ............ 47

Mechanism of Lipophilic Ion Transport . . . . . 47

Materials and Methods ........... 52

Results and Discussion ........... 56

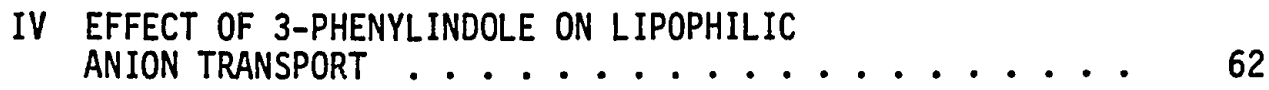

Mechanism of Transient Lipophilic Ion Transport . . . 62

Potential Energy Barriers for Lipophilic Ions . . . 66

Separation of Electrostatic and Non-electrostatic Effects on Ion Transport ......... 67

Materials and Methods............ 71

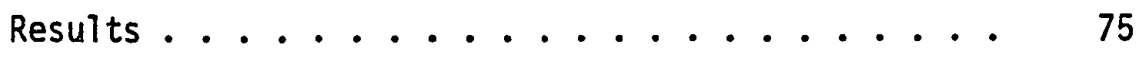

Discussion ........................ 81

$\checkmark$ ENHANCEMENT OF NONACTIN-K ${ }^{+}$STEADY STATE

TRANSPORT BY 3 -PHENYLINDOLE .......... 86

Mechanism of Nonactin Transport ........ 86

Materials and Methods............ 93

Results and Discussion ............... 95

VI EFFECT OF 3 -PHENYLINDOLE ON TRANSIENT NONACTIN-K ${ }^{+}$

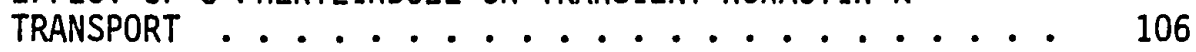

Transient Components of Nonactin- $\mathrm{K}^{+}$Transport . . . 106

Materials and Methods ........... 108

Results and Discussion .......... 109

VII CONCLUSION ................................ 113

Summary of Experimental Results . . . . . . 113 
CHAPTER

PAGE

Possible Biological Significance of the

Experimental Results ........... 115

Suggestions for Future Research . . . . . . . . 118

REFERENCES . . . . . . . . . . . . 122 


\section{LIST OF TABLES}

TABLE

PAGE

I Kinetic Parameters of Tetraphenylarsonium Transport in the Presence of 3-phenylindole ....... 58

II Partition Coefficient for Lipophilic Anions in the Presence of 3-phenylindole ......... 80

iII Comparison of Electrostatic and Non-electrostatic

Factors in Modification of Lipophilic Ion

Transport by 3 -phenylindole ..........

IV Kinetic Limitation Parameter for Nonactin- $\mathrm{K}^{+}$

Transport in the Presence of 3-phenylindole . . . . 104

V Relaxation Amplitude and Time Constant of Phosphatidylcholine/Cholesterol Bilayer in the Presence of Nonactin, $0.11 \mathrm{M} \mathrm{KCl}$ and $80 \mu \mathrm{M}$ 3-phenylindole ... 111 


\section{LIST OF FIGURES}

FIGURE

PAGE

1. Chemical formula of 3-phenylindole ......... 22

2. Surface pressure of $\mathrm{PC} /$ cholesterol monolayers as a

function of area per lipid molecule ....... 27

3. Adsorbed surface density of 3-phenylindole on to

$\mathrm{PC} /$ cholesterol monolayers as a function of aqueous

3-phenylindole concentration . . . . . . . 38

4. Experimental adsorption isotherm for 3-phenylindole . . 39

5. Surface potential of PC/cholesterol monolayers as a

function of area per lipid molecule ....... 41

6. Change in the surface potential as a function of

3-phenylindole concentration in the aqueous solution.

7. Change in the surface potential as a function of

adsorbed surface density of 3-phenylindole . . . . 43

8. Kinetic model of lipophilic ion transport . . . . . 48

9. Chemical formula of tetraphenylarsonium ....... 51

10. Schematic diagram of experimental set-up for measuring DC conductivity ......... 54

11. Tetraphenylarsonium zero voltage conductivity as a function of 3-phenylindole concentration . . . . 57

12. Potential change as a function of 3-phenylindole concentration .................... 60 
13. Chemical formulas of tetraphenylborate and dipicrylamine . 63

14. Activation energies involved in ion transport ..... 68

15. Block diagram of experimental set-up for measuring transient currents ........... 73

16. Lipophilic anion zero voltage initial conductivity as a function of 3-phenylindole concentration ..... 76

17. Zero voltage time constant for lipophilic anion transport as a function of 3-phenylindole concentration . . . 77

18. Adsorbed surface charge density and adsorbed surface number density as a function of 3-phenylindole concentration ................. 79

19. Chemical formula of nonactin .......... 87

20. Kinetic model of nonactin-K $\mathrm{K}^{+}$transport ........ 89

21. Theoretical curves of normalized conductivity as a function of voltage ................ 94

22. Nonactin $-\mathrm{K}^{+}$zero voltage conductivity as a function of 3-phenylindole concentration in the aqueous solution . 96

23. Relative conductivity as a function of 3-phenylindole

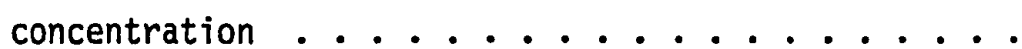

24. Nonactin $-\mathrm{K}^{+}$zero voltage conductivity as a function of $\mathrm{pH} . \ldots \ldots \ldots . . \ldots \ldots$

25. Nonactin- $\mathrm{K}^{+}$normalized conductivity as a function of applied voltage.......................... 100

26. Nonactin- $\mathrm{K}^{+}$zero voltage conductivity as a function of 3-phenylindole concentration in the membrane forming solution ............................ 103 


\section{CHAPTER I}

\section{INTRODUCTION}

\section{STRUCTURE AND FUNCTION OF BIOLOGICAL MEMBRANES}

In order to understand the mode of action of pesticides which influence cell membrane permeability, it is first necessary to understand the structure of the cell membrane and how it acts as a permeability barrier. Information on membrane structure and function is therefore covered in the first section of this chapter. The second section is a review of how the investigation of electrical conductivity induced by certain antibiotics and lipophilic ions was a stepping stone to the elucidation of ion transport in biological systems. Several pesticides which are known to influence cell membrane permeability are discussed in section three. Section four is a review of the research done by other investigators on the pesticide 3-phenylindole, while section five outlines the plan for the research I did on this same compound.

Every cell is surrounded by some type of membrane, and some intracellular organelles also are enclosed by a membrane. The composition of the membrane varies from cell type to cell type but the gross structural organization of mammalian membranes is along the lines described by Singer and Nicolson's fluid mosaic model (Singer and Nicolson, 1972). The fluid mosaic model, which is based on a thermodynamic consideration of hydrophobic and hydrophilic interactions, 
considers the membrane as primarily a lipid bilayer in which are embedded various prote ins at random locations. The 1 ip id bilayer is a stable configuration because lipid molecules are amphipathic; that is, they have both a nonpolar and a polar portion. Thus in the biological membrane the lipids are oriented with their polar headgroups facing the aqueous phase (cytoplasm or extracellular fluid), and the ir nonpolar fatty acid chains coming together to form the interior of the membrane.

The location of a given protein in or on the bilayer will be determined by the protein's three-dimensional configuration and by the amino acids on the protein's outer surface. A typical integral prote in would have nonpolar amino acid residues on the outer surface along most of its length, which is in contact with the fatty acid chains, and have polar or ionic residues at the ends, which protrude into the aqueous phase. Conversely, proteins normally found in the aqueous phases (e.g., dissolved in the blood stream) would have primarily hydrophilic residues on their outer surface. A peripheral prote in is a protein which is part of the membrane but which is removed essentially free of 1 ipids after only mild treatment; most membranebound glycoproteins are probably of this type. In summary, it should be emphasized that the lipid bilayer provides the ma in structure of the biological membrane and that the integrity of this bilayer must be maintained if the proteins are to be located in the ir normal locations.

The term fluidity is used to denote the physical state of the interior of the membrane. The fluidity or orderedness of a lipid 
bilayer is determined by the relative motion of the individual lipid molecules, which in turn is related to the length and degree of unsaturation of the fatty acid chains of the lipid. The evidence that the lipids in most membranes are in a fluid state, as proclaimed by the fluid mosaic model, comes from experimental studies utilizing $X$-ray diffraction, spin labeling, and differential scanning calorimetry (Singer and Nicolson, 1972; Lee, 1975). The fluidity of the membrane enables the various compounds to move about, which implies that a complete description of the biological membrane must be a dynamic one. The biogenesis of membranes is accomplished by movement of newly synthesized prote in and lipid components into their proper location, while catabolism is accompanied by removal of the products of degradation. Both lateral diffusion and transverse diffusion are involved in these two processes. The rate of flip-flop of lipid molecules across a pure lipid bilayer has been measured by both nuclear magnetic resonance and fluorescence spectroscopy (Kornberg and McConne11, 1971a and 1971b; Berden et al., 1975). The importance of membrane fluidity to the insertion and transport activity of proteins has been demonstrated in a study of the lactose permease protein of Escherichia coli (Saier and Stiles, 1975).

Cholesterol, an amphipathic molecule which exists in neutral form at physiological pH and is found in the membranes of nearly all mammalian cells, has a strong effect on fluidity and permeability of the membrane. It decreases the fluidity of lipid bilayers when the temperature is above the critical temperature of the lipids and increases the fluidity when the temperature is below the critical 
temperature of the lipids (Chapman, 1975). The description of the effect of cholesterol on lipid and lipid/protein mixtures has been an active area of investigation (Chapman and Penkett, 1966; Ladbrooke et al., 1968; Trauble and Sackman, 1972; Darke et al., 1972; Szabo, 1976).

According to the fluid mosaic model the lipid portion of the membrane is primarily in the form of a bilayer. The bilayer is two lipid monolayers back-to-back so some investigators have used lipid monolayers as a model to study the physical state of the bilayer. The pioneering work from which lipid monolayer research grew was the investigation of fatty acid, alcohol, and ester monolayers by Langmuir (1917). He was able to show conclusively that amphipathic molecules spread at an air/water interface form a layer one molecule thick and have a preferred orientation with respect to the interface. The experimenter has access to macroscopic variables such as surface tension, surface area, electrostatic potential, and temperature of the monolayer. The apparatus which enables one to measure isotherms of a monolayer, i.e. the relation betweel lateral pressure $\pi$ and area per molecule, is called a Langmuir trough in his honor. An excellent introduction to monolayer experimental technique and data interpretation is given by Gaines (1966), while more recent developments are covered by Goddard (1975).

Monolayers of amphipathic lipid molecules have been studied to determine lipid phase transition temperatures (Cadenhead, 1977; Albrecht et al., 1978; Nagle and Scott, 1978; Kellner et al., 1978), the effect of various headgroups (Phillips and Chapman, 1968; Paltauf 
et al., 1971; Phillips et al., 1972), and the effect of metal ions in the subsolution (Shah and Schulman, 1965; Papahadjapoulos, 1968). The condensing effect of cholesterol on phospholipid monolayers has been verified many times (van Deenan et al., 1962; Standish and Pethica, 1967; Shah and Schulman, 1967; Demel and Joos, 1968; Tinoco and McIntosh, 1970; Cadenhead et al., 1976).

Monolayers of lipids exist in several states depending on the area occupied per lipid molecule (Cadenhead, 1977). At very large area per molecule there is essentially no interaction between lipid molecules so this is called the gaseous state. At the other extreme, at small area per molecule, the lipid molecules are close enough that short range forces between fatty acid chains of the lipids and electrostatic forces between polar head groups become important. [The relative importance of these forces in interfacial phenomena is dealt with in an entertaining and informative manner by Israelachvili and Ninham (1977)]. In this situation only small changes in area per molecule induce a large change in $\Pi$ and the monolayer is said to be in a solid state. In between these two extremes is the liquid state which is further subdivided as to whether the monolayer is liquid expanded or liquid condensed. For phospholipids the region which is of most biological relevance is probably the liquid state. Phillips and Chapman (1968) pointed out that the transition from liquid expanded to liquid condensed in a phospholipid monolayer corresponds to the transition from liquid crystalline to gel in hydrated phospholipid bilayers. The latter transition can be seen in biological membranes as well as in lipid bilayer systems (Chapman, 1975). 
The prediction of the various transitions between states of the monolayer has been the goal of those working on the theory of lipid monolayers. The various theoretical approaches have been reviewed and evaluated by Wiegel and Kox (1980). One of the simplest approaches is to reduce the problem to two dimensions by mapping the chain conformations onto a lattice. This approach has the advantage that the equations are exactly solvable but it is inadequate to explain the features of experimental isotherms. The earliest equations of state for a liquid expanded monolayer were based on modifications of van der Waal's equation. Smith (1967) used a stack of hard disks of diameter $d$ as a model for the chains of fatty acid monolayers and derived the equation of state $\left[\pi+\pi E m d^{2} / 4 A^{2}\right]\left[A\left(1-\pi d^{2} / 4 A\right)\right]=k T$ where $I$ is the surface pressure, $E$ is the magnitude of the LennardJones potential energy when two disks just touch, $m$ is the number of disks (carbon atoms) in a chain, $A$ is the area per molecule, $k$ the Boltzmann constant, and $T$ the absolute temperature. Other modified van der Waal's equations have been proposed which incorporate several adjustable parameters and these can be brought into agreement with certain experiments but often the parameters have no physical theoretical foundation.

The main numerical methods used in theoretical studies of monolayers are Monte Carlo simulations based on a generation of random sequences of allowed states of the molecules and molecular dynamics, which deals with the interaction potentials among a large number of molecules. Yet another starting point for monolayer phase transition theory is the partition function. If the partition function contains 
several adjustable parameters then agreement between predicted and measured enthalpies is often satisfactory by this method. The equations of state deduced from a random walk analogy have so far been applicable only to the region of large area per molecule but in this region give qualitative agreement with experiment (Wiegel and Kox, 1980). The theories outlined so far have included only the interaction between lipid molecules yet it is certain that the contribution of the subphase (water and sometimes electrolyte) is not negligible. Gaines (1978) has attempted to incorporate the effect of water penetrating the monolayer following a Gibbs formalism with three adjustable parameters and concludes that the gradual exclusion of water from the monolayer is of paramount importance in the liquid expanded regime.

The study of the lateral pressure of lipid monolayers has thus been productive of theories even though the theories are still inadequate. Another property of lipid monolayers which is experimentally accessible for study is the change in electrical potential difference at the air/water interface $\Delta V$ caused by the presence of the Tipid monolayer. For a monolayer of neutral lipid it is thought that $\Delta V$ is due to the sum of three components: 1) reorientation and distribution of water dipoles around the lipid molecules, 2) the normal component of the permanent dipoles of the polar head group of the lipid molecules, and 3 ) the dipole due to the terminal group of the fatty acid chain. At present there is no theory to account for the first component; unfortunately there is also no way to experimentally measure the water component of $\Delta V$ separately from the other two components. However, the contribution from different portions of the polar head 
group (component 2) can be studied by varying the structure of the lipid molecule. One of the most important results to come from such an investigation was the finding of Paltauf, Hauser, and Phillips (1971) that the carbonyl parts of the ester linkage in the phospholipid molecule are a main cause of $\Delta V$ for that lipid. They found that substitution of an ether linkage for an ester linkage lowered $\Delta V$ by $30-200 \mathrm{mV}$. Shah and Schulman (1965) had neglected the ester linkage component but had pointed out that the glycerol portion and the phosphate/trimethylammonium group should contribute to $\Delta V$. Another way of investigating the contribution of the polar head group is a comparison between phosphatidylcholine (PC) and phosphatidylethanolamine (PE), which differ only in that PC has three methyl groups attached to the nitrogen whereas PE has three hydrogens attached to it. The $\Delta V$ for egg $P E$ is $40 \mathrm{mV}$ higher than $\Delta V$ for egg PC (H7adky, 1974), which probably reflects the different hydration and packing properties of the two lipids (Phillips, Finer and Hauser, 1972).

It has become conventional to express $\Delta V$ for neutral monolayers in terms of a surface dipole moment $\mu_{\perp}$ by the relation $\Delta V=K n_{\perp} / \varepsilon$, where $n$ is the number of lipid molecules per unit area, $\varepsilon$ is the dielectric constant which is often taken as unity, and $K$ is a constant whose value depends on the system of units. For 1 ipids in a liquid expanded state $\mu_{\perp}$ decreases as the area per molecule increases (Paltauf et al., 1971), suggesting that reorientation of film molecules is occurring. However, as mentioned above, it is likely that in this region water molecules are being excluded (Gaines, 1978) which could also account for a change in $\mu_{\perp}$. 
We will be concerned with a pesticide which is active against fungi and Gram-positive bacteria. The phosphoglycerides constitute a large fraction of total cellular lipids in fungi and bacteria (0'Leary, 1967). These may be acidic (e.g., phosphatidic acid, cardiolipin), negatively charged (e.g., phosphatidylserine, phosphatidylinositol, phosphatidylglycerol), or neutral (e.g., phosphatidylcholine, phosphatidylethanolamine) at physiological pH. Fungal membranes have a high proportion of the non-ionic phospholipids, phosphatidylcholine and phosphatidylethanolamine, whereas bacteria often contain phosphatidylethanolamine but almost never contain phosphatidylcholine (Ikawa, 1967). Fungi contain ergosterol and, in lesser amounts, several other sterols, but bacteria contain virtually no sterols (0'Leary, 1967). The fluid mosaic model outlined at the beginning of this chapter is mainly a model for the plasma and intracellular membranes of higher organisms. Fungi and bacteria have an inner membrane of this type but they also have an additional protective covering called the cell wall. In fungal cells and filaments the cell wall usually contains chitin, a polysaccharide. The bacterial cell wall is composed of peptidoglycan, whose backbone is a polymer of two amino acid sugars. The polymers are crossed linked by sequences of species-specific amino acids, resulting in a rigid three-dimensional structure. Most bacteria can be classified as either Gram-positive or Gram-negative based on a stain test, and the difference between the two types is found in the cell wa 11; Gram-positive bacteria have a much thicker cell wall than Gram-negative bacteria. The Gramnegative bacteria have an extra outer membrane of the fluid mosaic 
type, modified by the presence of 1 ipopolysaccharides (Nester et al., 1978; Davis et al., 1973).

\section{ION TRANSPORT IN BIOLOGICAL MEMBRANES AND LIPID BILAYERS}

One of the functions of the biological membrane is to act as a permeability barrier to the movement of ions. The permeability barrier is a potential energy barrier which depends primarily on the properties of the lipid bilayer portion of the membrane. A major component of the potential energy barrier is the work required to move an ion from bulk aqueous phase (dielectric constant $\varepsilon_{\mathrm{W}} \approx 80$ ) to bulk hydrocarbon (dielectric constant $\varepsilon_{\mathrm{m}} \approx 2$ ) as given by the Born energy $W_{b}=\left[q^{2} / 8 \pi \varepsilon_{0} r\right] \times\left[\left(1 / \varepsilon_{m}\right)-\left(1 / \varepsilon_{w}\right)\right]$, where $q$ is the charge of the ion, $r$ is the radius of the ion, and $\varepsilon_{0}$ is the permittivity of free space. The magnitude of the Born energy depends on the size of the ion, being greatest for small ions. Since the membrane has a finite thickness ( $\approx 50 \AA)$, polarization charges (image charges) will be induced at the interface giving an additional contribution to the potential energy of the ion. The combination of the Born energy and the image potential energy is the main contribution to the potential energy barrier for a small ion and collectively is called the electrostatic charging energy. Other components of the potential energy arise from: 1) the energy required to strip the ion of its hydration she11,2) electrostatic potential energy differences between aqueous phase and membrane interior due to lipid molecule dipole potential, and 3) short range (packing) interactions between the ion and the membrane (Andersen and Fuchs, 1975). Due to the existence of this potential energy barrier, 
the electrical conductivity of a lipid bilayer in the presence of electrolyte containing only inorganic ions is small, about $10^{-8}-10^{-7} \mathrm{~S} / \mathrm{cm}^{2}$.

The transport of solutes such as inorganic ions, sugars, and amino acids across the permeability barrier posed by the membrane is accomplished by several methods which fall into three general categories: 1) facilitated diffusion or mediated transport, an energy independent process, in which the solute is translocated by a prote in acting as a carrier or a channe 1, 2) group translocation, in which a chemical reaction occurs to alter the solute during translocation, and 3) active transport, which requires metabolic energy in the form of chemical energy or electrical energy. In Chapter VII we will be particularly interested in inorganic phosphate $\left(P_{j}\right)$ transport, which provides examples of all three categories. The $P_{i}$ transport out of mitochondria is often by an exchange process; for example, $P_{i}$ and malate can be carried in opposite directions by mediated transport (McGiven and Klingenberg, 1971). The superficially similar exchange of $\mathrm{P}_{\mathbf{j}}$ and $\mathrm{Cl}^{-}$in red blood cells is attributed by Bolis and Gomperts (1972) to simple diffusion. Inorganic phosphate can also be translocated by active transport in yeast (reviewed by Armstrong, 1972), in fungi (Lowendorf et al., 1974), and in mitochondria (Tyler, 1969; Coty et al., 1979). Phosphorylation during group translocation of sugars [e.g. in bacteria (Simoni, 1972)] must also be considered in connection with $P_{i}$ transport. An additional complication is that energy-dependent phosphate transport in one species of fungus, Neurospora crassa, has been shown to occur by a low affinity system, 
which operates under normal growth conditions, and a high affinity system which operates at a level dependent on phosphate concentration in the growth medium (Lowendorf et al., 1975; Burns and Beever, 1977).

To prove that a particular protein is functioning as a transport protein, it is necessary to isolate the prote in from the membrane under consideration and then reconstitute it into a lipid matrix formed of lipids of known composition and demonstrate transport activity. In general this worthy goal was not experimentally feasible until the latter part of the 1970s when new techniques for isolating proteins in active form were developed. Racker and his colleagues have been able to reconstitute parts of the mitochondrial, sarcoplasmic reticulum, and bacteriorhodopsin transport systems. For a partial listing of these accomplishments see, for example, Eytan, Matheson, and Racker (1976) and Racker (1979). Wohlrab (1980) has purified a prote in from mitochondria which, when reconstituted into liposomes containing a mixture of three lipids, was shown to exchange $P_{j}$ and also catalyze $P_{i}$ uptake. The reconstitution technique at the present time requires that the prote in be incorporated into 1 iposomes, and the ion transport followed by radioactive labeling of the ion or by indirect methods such as fluorescence spectroscopy. Direct electrical measurements cannot be made on these reconstituted systems. Since lipid and protein molecules are intermixed in the biological membrane, the interaction between lipids and proteins is a subject of immense interest; for instance, there is some evidence that membrane-bound enzymes and antigens require specific lipids for the expression of their activities (Triggle, 1970; Kagawa, Kandrach and Racker, 1973; Ragan and Racker, 
1973; Knowles and Racker, 1975). Reconstituted systems can be used to study some aspects of the interaction between proteins and lipids.

The beginnings of a physical theory for ion transport gradually emerged in the past two decades from investigations in a different direction, namely the study of the peptide antibiotics (e.g., polymixin, gramicidin, valinomycin) and polyene antibiotics (e.g., amphotericin, nystatin, filipin), which were found to influence the permeability of cell membranes. One category of antibiotics which affects the cell membrane includes valinomycin, the macrotetrolides (nonactin, monactin, dinactin, and trinactin), and enniatins, all of which were found by $X$-ray diffraction to form spherical or donut-shaped structures with many oxygen atoms pointing toward the center, where the ion resides, and the lipophilic groups pointing outward. Due to the ir low molecular weight and lipophilic envelope, the actins and valinomycin, unlike proteins, are easily dissolved in planar lipid bilayers. The investigation of the electrical properties of membranes was greatly accelerated by the introduction of an easy technique for the formation of planar 1 ipid bilayers (Mueller et al., 1963), in which electrodes could be placed on either side of the bilayer. The theory which emerged from experiments using these bilayer lipid membranes was that the antibiotics just mentioned act as carriers of alkali ions, picking the ion up on one side of the membrane, crossing the membrane as an ion/carrier complex, and then releasing the ion on the other side of the membrane. The carrier model was based on the Nernst-Planck electrodiffusion equation (Ciani, Eisenman and Szabo, 1969) or, alternatively, was developed using a chemical kinetic approach (Markin 
et al., 1969; Stark et al., 1971). In the sense that the transport requires a potential difference to exist across the membrane but does not require metabolic energy, the ion carrier mechanism demonstrated with antibiotics is analogous to facilitated diffusion in a biological membrane.

At the same time that the carrier mechanism was being elucidated, progress was being made in two other avenues of ion transport problems. The first avenue was the study of lipophilic ions and the second avenue was the study of channels. Lipophilic ions adsorb into deep potential energy minima at the aqueous/membrane interface and cross the membrane intact. Since there is no ion/carrier dissociation or recombination step, the transport scheme is much simpler than that of carrier mediated transport. This simplicity was very helpful in gaining a fundamental understanding of the processes of adsorption to the bilayer and subsequent translocation across the bilayer (Ketterer, Neumcke and Läuger, 1971). Thus lipophilic ions allow one to isolate one portion of the carrier mechanism.

It was recognized that the proteins in biological transport systems could conceivably function as either channels or carriers. Conventionally, a channel is a molecule or oligomer which stays in one location, spanning the membrane and allowing ions to pass through its hydrophilic interior. A carrier, on the other hand, is seen as a mobile molecule. Some antibiotics are clearly carriers as described above, and an unambiguous example of a channel was also found among the antibiotics in the form of the polypeptide gramicidin $A$. The evidence that gramicidin A forms a channel in planar lipid bilayers 
comes from two observations: when the antibiotic is present in smal1 amounts the membrane current fluctuates in a step-like manner as a constant voltage is applied across the membrane, and the rate of charge transfer is much greater than that due to a carrier mechanism (Hladky and Haydon, 1970). Ion transport in a channel has been described on the basis of Eyring rate theory as a series of thermally activated processes in which the ion moves from a binding site across an energy barrier to an adjacent site (Läuger, 1973). Recently, Läuger (1980) has given a theoretical treatment of a channel with multiple conformational states, and has shown that both channels and carriers are limiting cases of such a multistate channel.

After the basic transport mechanisms were verified, the ion carrier antibiotics and lipophilic ions became tools to probe how changes in membrane structure influence the ion transport process. Szabo et al. (1972) used ion/carrier complexes to show that ion transport is influenced by the composition of the lipid bilayer through the surface charges and surface dipoles of the lipids, and also through the fluidity of the bilayer interior. Stark et al. (1972) also used a carrier, valinomycin, to show that the electrical conductivity of phosphatidylcholine (lecithin) bilayers decreased with increasing length of the fatty acid chains of the lecithin. They concluded that the decreased conductivity was due to changes in the rate at which the membrane-bound carrier combines with an ion in the aqueous solution. This result was further verified by electrical relaxation experiments with valinomycin and attributed to chain dependent viscosity of the bilayer (Benz et al., 1973). The decrease in 
recombination with increasing chain length also occurs when the carrier is trinactin and the bilayer is formed from monoglycerides of various chain lengths (Benz and Stark, 1975). The influence of the polar head group was investigated by Benz et al. (1977), who found that monoglyceride allowed for a more stable ion/carrier complex than when the bilayer lipid was lecithin. They also reported that increasing the number of double bonds in the fatty acid residue increased the translocation rates of the complexed and uncomplexed carrier. Incorporation of cholesterol into monoglyceride bilayers decreases those two translocation rates and also the rate of recombination of ion/carrier complex (Benz et al., 1977). The rate of translocation of the ion/ carrier complex is nearly independent of bilayer thickness.

The lipophilic ions have also been used as probes to determine how membrane structure affects ion transport. Using the 1ipophil ic anion dipicrylamine, Benz and Läuger (1977) showed that the partitioning of the anion into the bilayer was unaffected by thickness of the hydrocarbon portion of the bilayer (solvent effect) but that the rate of translocation of the anion decreased with increasing thickness. The translocation rate was also influenced by' the head group; translocation occurred much faster in phosphatidylethanolamine bilayers than in lecithin bilayers, and much faster in lipids with ether linkage than in lipids with ester linkage between hydrocarbon chain and glycerol backbone. Increasing the number of double bonds in the fatty acid chains of lecithin decreases the amount of partitioning and increases the translocation rate of lipophilic anions (Benz and Läuger, 1977). In a comparative study of membrane structure using monoole in 
bilayers, the ion carrier complex $\mathrm{PV}-\mathrm{K}^{+}$, and the lipophilic anion dipicrylamine, Benz and Gisin (1978) found that the translocation rate of $\mathrm{PV}-\mathrm{K}^{+}$was much more sensitive to changes in chain length, number of double bonds, and nature of the polar head group, than was the translocation rate of dipicrylamine. They postulated that this is because the PV $-\mathrm{K}^{+}$adsorption plane is located more towards the hydrocarbon region of the bilayer while that of dipicrylamine is more toward the headgroup and aqueous region. For both monoglyceride and lecithin bilayers, the translocation rate constant increases with decreasing fatty acid chain length whereas increasing the number of double bonds increases the translocation rate and decreases the partition coefficient (Benz and Gisin, 1978). The partition coefficient for the positively charged $\mathrm{PV}-\mathrm{K}^{+}$complex is much less than for the negatively charged dipicrylamine when the bilayer is composed of the negatively charged lipid phosphatidylserine and the ionic strength of the aqueous phase is very high (Benz and Gisin, 1978).

\section{DISRUPTION OF MEMBRANE STRUCTURE AND FUNCTION BY PESTICIDES}

In his eternal quest for dominion over the earth, man has discovered and, in this century, synthesized an enormous variety of compounds which alter the growth and functioning of other species. Among these compounds are types used to control fungi (fungicides), to eliminate weeds (herbicides), to incapacitate insects (insecticides), and to destroy rodents (rodenticides). A major goal of the study of all such pesticides is to understand their mode of action on a molecular basis and ultimately to develop a theoretical framework 
for the design of new pesticides with the desired potency and maximum specificity. On a biological and biochemical level, the mode of action of pesticides which interfere with photosynthesis, respiration, or acetylcholinesterase activity is relatively well understood, but the mode of action is much less clear for those pesticides thought to act in areas where the fundamental biochemistry is still obscure, e.g. pesticides believed to interfere with cell division, axonal transmission, or growth processes in plants (Corbett, 1974). In view of the importance of lipids in maintaining structural organization of the cell membrane, it is not surprising that some pesticides are effective because they physically disrupt membrane structure. Methyl decanoate, a fatty acid derivative, causes such severe damage to the membranes of plant buds, which lack a cuticle covering, that the damage is clearly seen in electron micrographs of treated tissues (Nelson and Reid, 1971). Electron micrographs have also proven that the bipyridylium herbicide paraquat causes rupture of the tonoplast membrane surrounding leaf vacuoles (Dodge, 1971) leading to an increased permeability (Dodge, 1971; Merkle et al., 1965). Another pesticide which disrupts membrane structure is petroleum oils used to control mites, weeds, and banana leaf fungus; the petroleum oils probably act by solubilizing the lipid portion of the membrane (Corbett, 1974).

We now discuss several antibiotics which may serve as a model for pesticides such as those mentioned in the preceding paragraph which cause structural damage to the membrane. Polymyxin, a polypeptide antibiotic which causes leakage of cell constituents in both growth 
and non-growth conditions (Newton, 1956), is more active against Gramnegative bacteria than against Gram-positive bacteria (Zähner and Maas, 1972) and seems to specifically interact with phosphatidylethanolamine (Davis et al., 1973). In an investigation of the site of action of polymyxins, it was found that the polymyxins adsorb much more strongly to phospholipids of sensitive bacteria than to phospholipids of resistant bacteria (Zähner and Maas, 1972). Thus it appears that for these antibiotics, interaction with phospholipids or the lipid portion of lipopolysaccharides is a prerequisite for cell permeability changes. Another group of antibiotics which grossly alter the permeability of the cell membrane is the polyene antibiotics (e.g., nystatin, amphotericin B, filipin), which cause a severe loss of cytoplasmic constituents (Kinsky, 1961; Marini, Arnow and Lampen, 1961) and whose site of action has been localized to the sterol component of the membrane, whether cholesterol or ergosterol (Lampen et al., 1960; Kinsky, 1962; Deme 1, van Deenen and Kinsky, 1965). The physicochemical mechanism of the action of polyenes was investigated in more detail by monitoring the electrical properties of sterol-containing bilayer lipid membranes in the presence of the antibiotics. Andreoli and Monahan (1968) found that filipin caused extreme membrane instability while nystatin and amphotericin $B$ decreased the electrical resistance of the membranes by as much as six orders of magnitude. Finkelstein and Cass (1968) demonstrated that the membranes became slightly anion selective in the presence of the polyenes and that increasing temperature greatly decreased the electrical conductance. Both groups noted that the electrical conductance varied as a high power (4.5 or 10) of the 
antibiotic concentration and therefore hypothesized that the polyenes bind to sterols and in some fashion aggregate to form pores in the membrane.

Several types of insecticides, fungicides, and herbicides have also been investigated by the technique of measuring changes in electrical conductivity of bilayer lipid membranes. This technique has proven especially useful in the elucidation of the mechanism of action of uncouplers, that is, compounds which uncouple electron transport from adenosine triphosphate synthesis by dissipating the hydrogen ion electrochemical potential gradient in mitochondria or chloroplasts. Salicylanilides, carbonylcyanide phenylhydrazones, 2,4-dinitophenol, and chloro-substituted phenols are examples of such uncouplers (Liberman and Topaly, 1968; Bielawski et al., 1966; Smejtek, Hsu and Perman, 1976). Lea and Croghan (1969) and, independently, Finkelste in (1970) proposed a carrier model for the transport in which the uncoupler anion combines with a hydrogen ion at the membrane/aqueous interface, crosses the membrane in the form of a dimer, and releases the hydrogen ion at the opposite interface, resulting in a net proton flux. This mechanism is reviewed by McLaughl in and Dilger (1980), who also compare the action of protonophores on artificial membranes and on mitochondrial membranes.

Another compound whose effect on conductivity of bilayer lipid membranes has been characterized is the widely used herbicide 2,4-dichlorophenoxyacetic acid (2,4-D). The site of action of 2,4-D is unknown but its primary effect is on plant growth and it is believed to interfere with the proper functioning of indole-3-acetic acid, a 
naturally occurring plant hormone (Corbett, 1974). The neutral form of 2,4-D enhances the transport of positively charged ions (tetraphenylarsonium and nonact in $-\mathrm{K}^{+}$) and suppresses the transport of tetraphenylborate, a negatively charged ion (Smejtek and Paul isIllangasekare, 1979a and 1979b). The significance of this fact in relation to the biological effect of $2,4-D$ is still not clear but, nevertheless, the phenomenon is intriguing and it can be explained on a physical basis. Smejtek and Paulis-Illangasekare (1979a; 1979b) propose that a layer of 2,4-D molecules is formed at the aqueous/ membrane interface, probably on the membrane side. Since 2,4-D has a net dipole moment, the layer changes the electrostatic energy barrier in the interior of the membrane, making the passage of positively charged ions less probable. Thus in the case of 2,4-D the pesticide exerts its effect on membrane permeability indirectly by electrostatic interactions rather than directly acting as a carrier or pore.

\section{EFFECT OF 3-PHENYLINDOLE ON FUNGAL CELL MEMBRANE STRUCTURE AND FUNCTION}

We turn now to consideration of 3-phenylindole (3PI), an antimicrobial compound from the group of substituted indoles which is active against many fungi and Gram-positive bacteria (Dekker et al., 1975; Hoppe et al., 1976a). The chemical structure of 3PI is shown in Fig. 1. In a search for the mode of action of 3PI, Hoppe, Kirkenaar, and Sijpesteijn (1976a) [abbreviated hereafter as HKSa] found that 3PI exerted a fungistatic rather than a fungicidal effect on Aspergillus niger and that growth inhibition could be neutralized by addition of phospholipids. They also report that 3PI markedly affects 


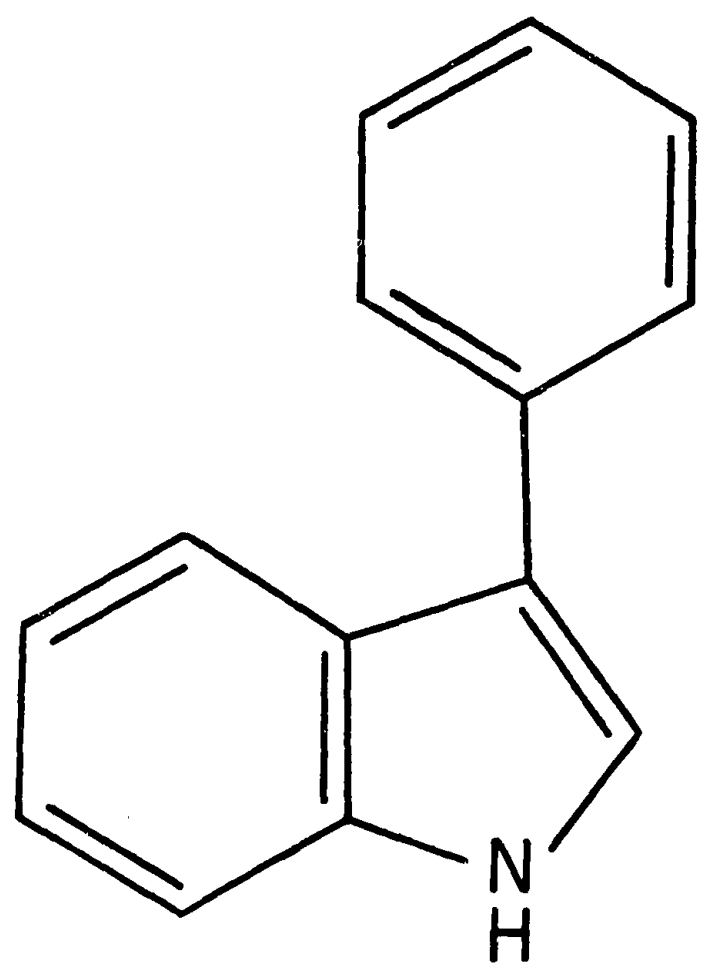

Figure 1. Chemical formula of 3-phenylindole. 
the composition of the lipid fraction of $\underline{A}$. niger, causing a decrease in total phospholipid content and an increase in free fatty acids. Phosphatidylethanolamine and phosphatidylcholine are the main phospholipids of $\underline{A}$. niger, with lesser amounts of diphosphatidylglycerol, phosphatidylinositol, phosphatidylserine, and phosphatidic acid. HKSa showed that all of these phospholipids decreased in a similar way but that the sterol component was not affected.

To determine whether 3PI causes a general increase in permeability similar to that induced by methyldecanoate, paraquat, polymyxins, and the polyene antibiotics, HKSa measured the leakage of ${ }^{32} \mathrm{p}-\mathrm{labeled}$ compounds from $\underline{A}$. niger. They found a slight leakage under growth conditions but not in non-growth conditions, in contrast to polymyxin which caused leakage in both circumstances. While 3PI apparentiy does not change the cell membrane permeability to the extent that polymyxin and the polyene antibiotics do, it does affect the uptake of several biosynthetically important molecules. By monitoring the radioactivity due to $\left[{ }^{32}\right.$ p]orthophosphate, Hoppe, Kirkenaar, and Sijpesteijn (1976b) [abbreviated hereafter as HKSb] showed that $3 P I$ reduces the incorporation of ${ }^{32} \mathrm{P}_{i}$ into phospholipids and greatly inhibits the uptake of ${ }^{32} \mathrm{P}_{i}$ by $\underline{A}$. niger mycelium. They report that 3PI causes a smaller effect on the incorporation and uptake of $\left[{ }^{14} \mathrm{C}\right]-$ phenylalanine, a protein precursor, and $\left[{ }^{14} \mathrm{C}\right]$ uridine, a nucleic acid precursor.

The uptake of 3PI itself was also investigated by HKSa, who report that uptake of $3 P I$ into $\underline{A}$. niger mycelium was rapid, being detectable within ten minutes. In addition, HKSa present spectroscopic 
evidence that $3 \mathrm{PI}$ binds to phospholipids in vitro. They found that the two peaks in the ultraviolet spectrum of 3PI dissolved in $50 \mathrm{mM}$ $\mathrm{KCl}$ were shifted to longer wavelengths in the presence of phospholipid liposomes and that the $\mathrm{NH}-$ band $\left(3400 \mathrm{~cm}^{-1}\right)$ in the infrared spectrum of 3PI showed a strong decrease in intensity in the presence of dry phospholipids. In a related experiment HKSa produced a mutant strain of $\underline{A}$. niger which was resistant to the fungistatic action of $3 P I$ and, using thin layer chromatography for the detection of 3PI, showed that 3PI accumulated in the chloroform-soluble portion of mycelium of the resistant fungus strain to the same extent as in the wild strain. Recall that polymyxin showed a preferential adsorption to phospholipids of sensitive bacteria as discussed earlier.

It is interesting to compare the mode of action of $3 P I$ with the mode of action of pantoyl lactone. Johnson et al. (1980) reported that the latter compound changes the phospholipid and fatty acid pattern of Micrococcus lysodeikticus, and that significant amounts of the two major phospholipids of $\underline{M}$. lysodejkticus (phosphatidylglycerol and diphosphatidyiglycerol) are converted to lyso form. They also found that pantoyl lactone inhibits the uptake of three amino acids (D-alanine, L-glutamic acid, L-aspartic acid) but that the inhibition is reversible since it is completely removed when the cells are transferred to a pantoyl lactone-free medium. Thus, 3PI and pantoyl lactone provide examples of compounds which interact with cell membrane phospholipids and interfere with transport into or across the membrane. 


\section{PROJECT DESCRIPTION}

The goal of the present research is to clarify the physical effects of the pesticide 3-phenylindole (3PI) on the transport induced in bilayer lipid membranes; additional information on 1ipid/3PI interaction is obtained through the use of lipid monolayers. A general overview of the methodology is presented here while details of each type of experiment are presented in Chapters II through VI. Experimental conditions in the present work have been chosen to follow closely the conditions of the more biologically oriented investigations of HKSa and HKSb discussed in the preceding section. Thus the salt concentration of aqueous solutions is a tenth molar and the $\mathrm{pH}$ in most of the conductivity experiments is 6.9. The 3PI is added from acetone, and the final aqueous concentration is varied from 0.5 to $80 \mu \mathrm{M} 3 \mathrm{PI}$, a range which includes the concentrations used by HKSa and HKSb. The model membranes used in the present study are either monolayers or bilayers consisting of phosphatidylchol ine and cholesterol, with cholesterol content at a mole fraction of 0.22 . Although the interaction of 3PI in the fungus was only with phospholipids, cholesterol is included in our bilayers to increase their stability, and in our monolayers so that monolayer and bilayer results can be more easily correlated. Note that in what follows we use the term 'lipid' in its broader sense to include both cholesterol and phospholipid.

HKSb showed by spectroscopic methods that 3PI binds to phospholipids. In this study we use the interaction between 3PI and lipid monolayers to quantify the amount of pesticide uptake or adsorption. The monolayer studies also give some indication of the location of the 
adsorbed 3PI relative to the aqueous/lipid interface. Furthermore, use of lipid monolayers allows direct measurement of changes in the interfacial potential, which is important to the problem of transport of ions and ionophores across bilayers.

The leakage of ${ }^{32} \mathrm{p}$-labeled compounds and the inhibition of the uptake of three biosynthetic precursors as determined by HKSa and HKSB led them to suggest that 3PI may affect transport processes in fungal mycelium. We investigate the possibility that 3PI can alter ion transport in the absence of any metabolic processes by measuring the conductivity of bilayer lipid membranes in the presence of 3PI. We utilize two kinds of conductivity measurements. The first kind is measurement of steady state conductivity, which involves the application of a constant voltage to the membrane and measurement of the current present after several seconds. The second kind is measurement of transient currents, which involves the application of a voltage step across the membrane and continuous monitoring of the current from a few microseconds to, for instance, several milliseconds after the voltage is applied. In principle more information on the kinetics of transport is available from measurements of transient currents. For example, by the transient current technique, it is possible to distinguish between changes in the amount of lipophilic ion which adsorbs to the membrane from changes in the rate of translocation. Steady state conductivity measurements do not allow such a distinction to be ascertained.

It is possible that 3PI could affect ion transport not directly by acting as a carrier or pore but indirectly by changing the 
environment in or near the membrane. We examine this hypothesis using probes of two different types, namely a carrier (nonactin) and a lipophilic ion (tetraphenylarsonium). When a voltage is applied across the membrane, the lipophilic ions adsorb to the membrane, cross the membrane, and desorb from the opposite side of the membrane. The carrier mechanism, however, involves several other steps (combination with the ion, dissociation from the ion, diffusion of the neutral carrier) in addition to translocation of the ion/carrier complex across the membrane. We compare the effect of 3PI on the transport of these two types of probes to determine whether 3PI affects any of the steps specific to the carrier mechanism.

The indirect means by which 3PI could influence ion transport can be divided into two categories, electrostatic and nonelectrostatic. We test the possibility that 3PI causes changes in the electrostatic energy barrier by measuring changes in the electrical conductivity of bilayer lipid membranes doped with either positively (nonactin- $\mathrm{K}^{+}$, tetraphenylarsonium) or negatively (tetraphenylborate, dipicrylamine) charged probes. If the primary effect of 3PI were to change the height of the electrostatic energy barrier in the middle of the membrane, we would expect 3PI to influence the conductivity due to positively and negatively charged probes to about the same extent but in opposite directions, e.g., decreasing the conductivity due to positively charged probes and increasing the conductivity due to negatively charged probes.

Tetraphenylborate (see Fig. 13) was our original choice for the negatively charged ion since it is structurally very similar to 
positively charged tetraphenylarsonium (see Fig. 9). However the results of the experiment with tetraphenylborate were so unexpected that we thought it necessary to use a second negatively charged ion to verify them. Thus, transient current measurements are also done using dipicrylamine (see Fig. 13) whose chemical structure is sufficiently different from that of tetraphenylborate that the possibility of a specific interaction between the ion and the pesticide could be investigated. As mentioned earlier, more information is available from transient current measurements than from steady state measurements, and thus it would have been preferable to examine the transient currents due to both positively and negatively charged lipophilic ions. However, the magnitude of the transient current due to tetrapheny?arsonium is too small to be measured by our instrumentation and so only the anions could be investigated by the transient technique. 


\section{CHAPTER II}

\section{THE EFFECT OF 3-PHENYLINDOLE ON LIPID MONOLAYERS}

\section{ADSORPTION TO LIPID MONOLAYERS}

The use of lipid monolayers as a model to study the physical state of lipid membranes was discussed in the first section of Chapter I. In this chapter we are concerned with changes in the monolayer when the pesticide 3PI is present in the aqueous solution in contact with the lipid monolayer. In order to understand the influence of a pesticide on membrane properties and on ion transport across membranes it is necessary to know how much of the pesticide which is initially in the fluid surrounding the cell or organism actually enters the membrane phase. The partitioning of lipophilic molecules or ions between the bulk aqueous phase and the lipid phase depends linearly on the aqueous concentration $c$ of the lipophilic molecule or ion for small values of $c$. That is, $\Gamma=\beta c$ where $\Gamma$ is the number density of molecules adsorbed onto the lipid and $\beta$ is a constant called the partition coefficient. The coefficient $\beta$ has units of distance and can be thought of as the thickness of aqueous solution which would contain the same amount of pesticide as is adsorbed to the membrane. At higher values of $c$ the number of available adsorption sites puts a limit on the extent of adsorption and $\Gamma$ is no longer a linear function of $c$. This situation is often described well by a Langmuir

adsorption isotherm $\left[(1 / \Gamma)-\left(1 / \Gamma_{m}\right)\right]^{-1}=\beta_{L} c$ where $\Gamma_{m}$ is the maximum 
adsorbed surface density and $\beta_{L}$ denotes the partition coefficient for a Langmuir adsorption isotherm (Delahy, 1965; deLevie et al., 1979). There are several experimental methods to obtain values for $\Gamma_{m}$. For instance, Rich (1973) calculated the adsorption isotherm for the dissociated form of dinitrophenol, an uncoupler of oxidative phosphorylation, using microelectrophoresis measurements. DeLevie et al. (1979) used conductivity data on several probe ions to derive an adsorption isotherm for the neutral form of phloretin, an inhibitor of hexose and chloride transport in red blood cells. In the present study we have used monolayer techniques to obtain an adsorption isotherm for 3PI onto lipid monolayers and by fitting the data to a Langmuir adsorption isotherm, which can be written in the form

$$
\frac{1}{\Gamma}=\frac{1}{\beta_{L}} \cdot \frac{1}{C}+\frac{1}{\Gamma_{m}}
$$

have obtained values for the partition coefficient $\beta_{L}$ and the maximum adsorbed surface density $\Gamma_{m}$.

The change in surface tension of an air/water interface due to the presence of a lipid monolayer is conventionally called the surface pressure $\pi$; i.e. $\pi=\gamma_{0}-\gamma_{2}$ where $\gamma_{2}$ is the surface tension when the monolayer is present and $\gamma_{0}$ is the surface tension when it is absent. In the experiment the area in which the monolayer is contained is varied so that $\pi$ is measured as a function of area per lipid molecule $A_{2}$. The value of $A_{2}$ was obtained by dividing the area of the monolayer by the total number of lipid molecules spread.

We found that 3PI did not form a compressible monolayer at the air/water interface. This is not surprising in view of the fact that according to the structure shown in Fig. 1 there is no reason to 
suspect 3PI would be amphipathic. However 3PI does adsorb to a lipid monolayer in such a way as to increase the apparent area per lipid molecule at any given $\pi$. Based on simple geometrical considerations, the change $\Delta A_{2}$, in the apparent area per lipid molecule at a given $\pi$ is given by $\Delta A_{2}=A_{2}-A_{0}$ where $A_{2}$ is the apparent area per lipid molecule in the presence of $3 P I$ and $A_{0}$ is the area per lipid molecule in the absence of 3PI. If we denote the area of a 3PI molecule by $A_{1}$, assumed independent of $\pi$, then $\Delta A_{2} / A_{1}$ must be the number of $3 P I$ molecules per lipid molecule. Since the number density of 1 ipid molecules is $1 / A_{2}$, the number density, $\Gamma$, of $3 P I$ molecules can be expressed as

$$
\Gamma=\Delta A_{2} /\left(A_{1} \cdot A_{2}\right)
$$

\section{ELECTROSTATIC POTENTIAL CHANGES DUE TO ADSORBED 3-PHENYLINDOLE}

The presence of an air/water interface creates an electrostatic potential difference $V_{0}$ between the bulk water phase and the bulk air phase because the highly polar water molecules are not randomly oriented at the interface. The change in this electrostatic potential due to the presence of a lipid monolayer is called surface potential $\Delta V$, i.e. $\Delta V=V_{2}-V_{0}$ where $V_{2}$ is the potential in the presence of a monolayer. All potentials are with respect to the bulk aqueous phase. The presence of adsorbed 3PI molecules causes an additional change in the potential $\delta(\Delta V)$ defined as

$$
\delta(\Delta V)=\Delta V_{1,2}-\Delta V_{2},
$$

where $\Delta V_{1,2}$ is the surface potential when $3 P I$ is in the aqueous 
solution and $\Delta V_{2}$ is the surface potential of the lipid monolayer in the absence of $3 P I$. Such a change in the electrical potential has important implications for ion transport and will be discussed in more detail in Chapter IV. The change in surface potential when 3PI is in the aqueous phase is due to: 1) the adsorption of 3PI molecules each having a dipole moment with normal component $\mu_{\perp}$, and 2) a decreased density of lipid molecules. Neglecting any reorientation of lipid or water molecules, the change in surface potential is, therefore,

$$
\delta(\Delta V)=12 \pi \Gamma \mu_{\perp} / \varepsilon-\frac{\Delta A_{2}}{A_{2}} \Delta V_{2},
$$

where $\varepsilon$ is the dielectric constant in the region of the 3PI adsorption plane. The second term in Eq. (4) can also be expressed in terms of $\Gamma$ through Eq. (2) in order to obtain the following relationship between $\delta(\Delta V)$ and $\Gamma$ :

$$
\delta(\Delta V)=\left(12 \pi \mu_{\perp} / \varepsilon-\Delta A_{1} \Delta V_{2}\right) \cdot(\Gamma) \quad .
$$

By fitting our data to Eq. (5), we obtain a value for $\mu_{\perp}$ from the slope of the line; if $\delta(\Delta V)$ is in volts and $\Gamma$ is in molecules $/ \AA^{2}$, then $\mu_{\perp}$ will be in Debye.

\section{MATERIALS}

L- $\alpha$-phosphatidylcholine (PC) from egg yolk (Sigma Chem. Co., St. Louis, MO) showed a single spot by thin layer chromatography and was used without further purification. Recrystallized, Tyophilized cholesterol was a gift of Dr. D. McClure of the Chemistry Department. The spreading solvents, chloroform and hexane, were of the highest 
quality (Matheson, Coleman and Bell; Omnisolv grade). Both analytical grade acetone (Amachem, Portland, OR) and chromatography grade acetone (Mallinckrodt, St. Louis, MO) affected the $\pi-A_{2}$ and $\Delta V-A_{2}$ curves to the same extent so the A.R. grade was routinely used. Potassium chloride (Mallinckrodt, A.R.) and distilled deionized water from a Millipore Q2 system (Millipore Corp., Bedford, MA) were used for all aqueous solutions.

3-phenylindole was synthesized following the method of Fischer and Schmidt (1888) with minor modifications. Phenylhydrazine (MC/B) was added in equimolar quantity to $100 \mathrm{~g}$ ( 0.83 mole) phenylacetaldehyde (Aldrich technical grade), heated in a steam bath for an hour and then diluted with about $300 \mathrm{ml}$ absolute alcohol. To the mixture was added $200 \mathrm{ml}$ of absolute alcohol which had been saturated with hydrogen chloride gas. The solution was then refluxed in nitrogen environment for 45 minutes, again using the steam bath. When the solution had cooled to room temperature, enough water ( $1500 \mathrm{~m} 1$ ) was added to dissolve the ammonium chloride and the excess hydrazine salts. The solution was filtered several times, discarding the liquid at each step. The precipitate was dried in a dessicator in the presence of $\mathrm{KOH}$ pellets for 90 minutes. The product was then recrystallized from toluene/hexane since it is very soluble in toluene and less soluble in hexane. After sublimation the product was granular, ivory color, and had a distinctive, not unpleasant, odor. The melting point was $86.5-87.5^{\circ} \mathrm{C}$, in agreement with published values (Fischer and Schmidt, 1888; Dekker, Selling and Overeem, 1975). 
PROCEDURE

$\pi-A_{2}$ and $\Delta V-A_{2}$ curves were obtained with a fully automated Langmuir trough. Surface pressure was monitored by a glass Wilhelmy plate coupled to a linear differential transformer (Automatic Timing and Control, Inc., King of Prussia, PA) whose output was fed to an $X-Y$ recorder (Moseley 7035A). The electrical potential between a calomel electrode in the aqueous solution and a radioactive polonium electrode (Nuclear Products, El Monte, CA) about three millimeters above the water was measured by a fast voltmeter (response time $\sim 1$ msec) whose output was fed to an $X-Y$ recorder (Moseley 7035B). The voltmeter was designed using an ultra-low bias current ( $\sim 0.1$ PA) FET operational amplifier (BB3523) as a buffer to obtain input impedance of the order of $10^{13} \Omega$. The Teflon trough $(29 \mathrm{~cm} \times 15.2 \mathrm{~cm}$; depth $1.2 \mathrm{~cm})$ was bolted to a thick slab of brass to help it retain its shape. The monolayer area was determined by the position of two Teflon barriers of which one was stationary and the other motor-driven. To minimize the problem of electrostatic charge build-up (Costa et al., 1976) the barriers were passed close to the radioactive source immediately after cleaning.

Cleanliness of the system was maintained in several ways. The Teflon trough and barriers were soaked every few days in concentrated sulfuric acid:nitric acid 9:1 and then rinsed extensively with deionized water. The Wilhelmy plate was etched initially with hydrofluoric acid. Between each run the plate was wiped clean with ethanol and then rinsed successively in ethanolic $\mathrm{KOH}, \mathrm{H}_{2} \mathrm{O}, 1 \% \mathrm{HCl}$, and then more $\mathrm{H}_{2} \mathrm{O}$. The surface of the aqueous solution was wiped clean between 
each run with lens cleaning tissue; absence of a change in both pressure and potential upon maximum compression was taken as proof of a clean surface.

Since the spreading solvents were highly volatile, precautions were taken to ensure an accurate value for the lipid concentration. Cholesterol and PC were dried and a mixture of chloroform:hexane 1:1 added to give a stock solution with cholesterol at a mole fraction of $Y=0.22$. Each day a portion of the lipid stock was evaporated to dryness under nitrogen in a $1 \mathrm{ml}$ vial, weighed, made up to $1 \mathrm{ml}$ with the mixed solvent, and then diluted with the same solvent mixture to give a final lipid concentration of $1.00 \mathrm{mg} / \mathrm{ml}$. The bottle with the dilute solution was placed in a jar which had a layer of solvent mixture in the bottom and had become saturated with the solvent vapor. Thus evaporation from the lipid solution was minimized.

The aqueous solution was prepared fresh each day. Since 3PI is surface active, it was necessary to inject 3PI in acetone beneath the surface of a $\mathrm{KCl}$ solution which was being constantly stirred. Since acetone affected the surface potential, the amount of acetone in all aqueous solutions, both with and without 3PI, was held constant at $0.5 \%$.

Lipid from the dilute solution was dropped by microliter syringe onto the aqueous solution. After a period of about 15 minutes to allow the solvent to evaporate, the monolayer was compressed at a rate of about $6 \AA^{2} /$ molecule/minute. The average of at least four runs for each 3PI concentration was used in calculations. All measurements were done at room temperature. The system was checked by taking 
$\pi-A_{2}$ and $\Delta V-A_{2}$ curves for monolayers of a single component legg $P C$; cholesterol) and it was found that these curves were consistent with published results (Shah, 1970; Hilton and O'Brien, 1973; Demel and Joos, 1968; Papahadjapoulos et al., 1973). Pautauf et al. (1971) found that $\mu_{\perp}$ for both distearoy 1 PC and distearyl PC decreased with decreasing area per molecule. We found a similar phenomenon for egg PC; a plot of $\mu_{\perp}$ as a function of area per lipid molecule had a slope of $3.5 \times 10^{-3}$ Debye $/ \AA^{2}$ per molecule and a value for $\mu_{\perp}$ of 0.68 Debye at $60 \AA^{2}$ per molecule, taking the dielectric constant as unity.

\section{RESULTS}

Representative $\pi-A_{2}$ curves of $P C /$ cholesterol monolayers in the presence of various concentrations of $3 P I$ are shown in Fig. 2. Accuracy of $\pi$ was 0.2 dyn $/ \mathrm{cm}$ and reproducibility was usually within 1.5 $\AA^{2}$. The area per molecule was obtained by dividing the area of the monolayer by the total number of PC plus cholesterol molecules spread. The presence of 3PI caused a shift of the curves toward greater area per molecule and a change in the slope of the curves:

Using the $\pi-A_{2}$ curves the number density of adsorbed 3PI molecules at 40 dyn/cm was calculated at each 3 P I concentration using Eq. (2) and the results shown graphically in Fig. 3. The area $A_{1}$ of a 3PI molecule was taken as $42.8 \AA^{2}$ based on the projected area of a Corey-Pauling-Koltun model. Error bars are the standard deviation for at least four monolayers. At low [3PI] $\Gamma$ increases with [3PI] but at higher [3PI], I becomes nearly independent of [3PI]. This is characteristic of Langmuir type adsorption and Fig. 4 displays this 


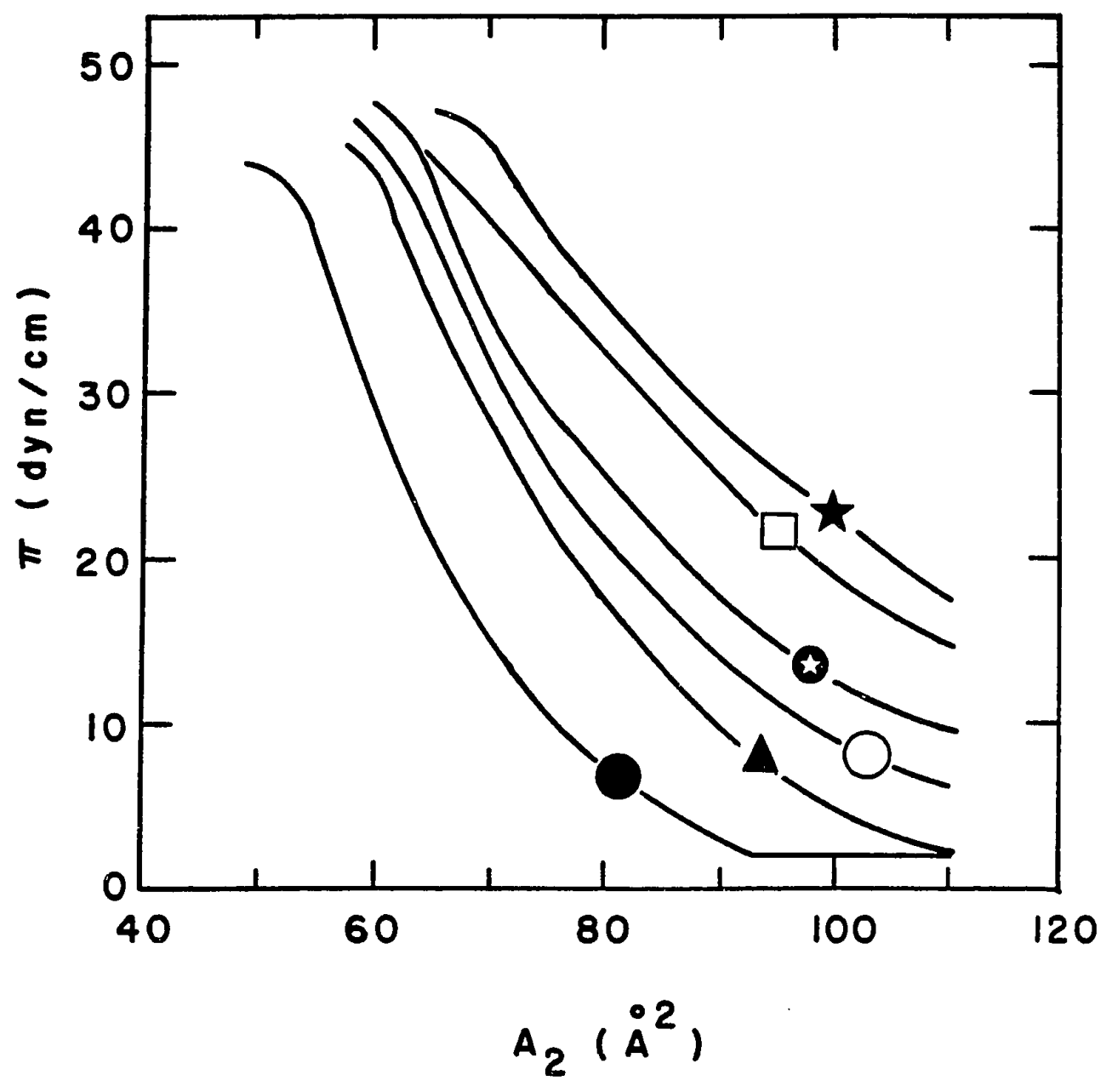

Figure 2. Surface pressure of phosphatidylcholine/cholesterol monolayers as a function of area per lipid molecule. Aqueous solution

$0.11 \mathrm{M} \mathrm{KCl}$ plus the indicated concentration of 3-phenylindole:

$0 \mu \mathrm{M}, \triangle 5 \mu \mathrm{M}, \mathrm{O} 15 \mu \mathrm{M}, \bigotimes 25 \mu \mathrm{M}, \square 40 \mu \mathrm{M}, \star 70 \mu \mathrm{M}$. 


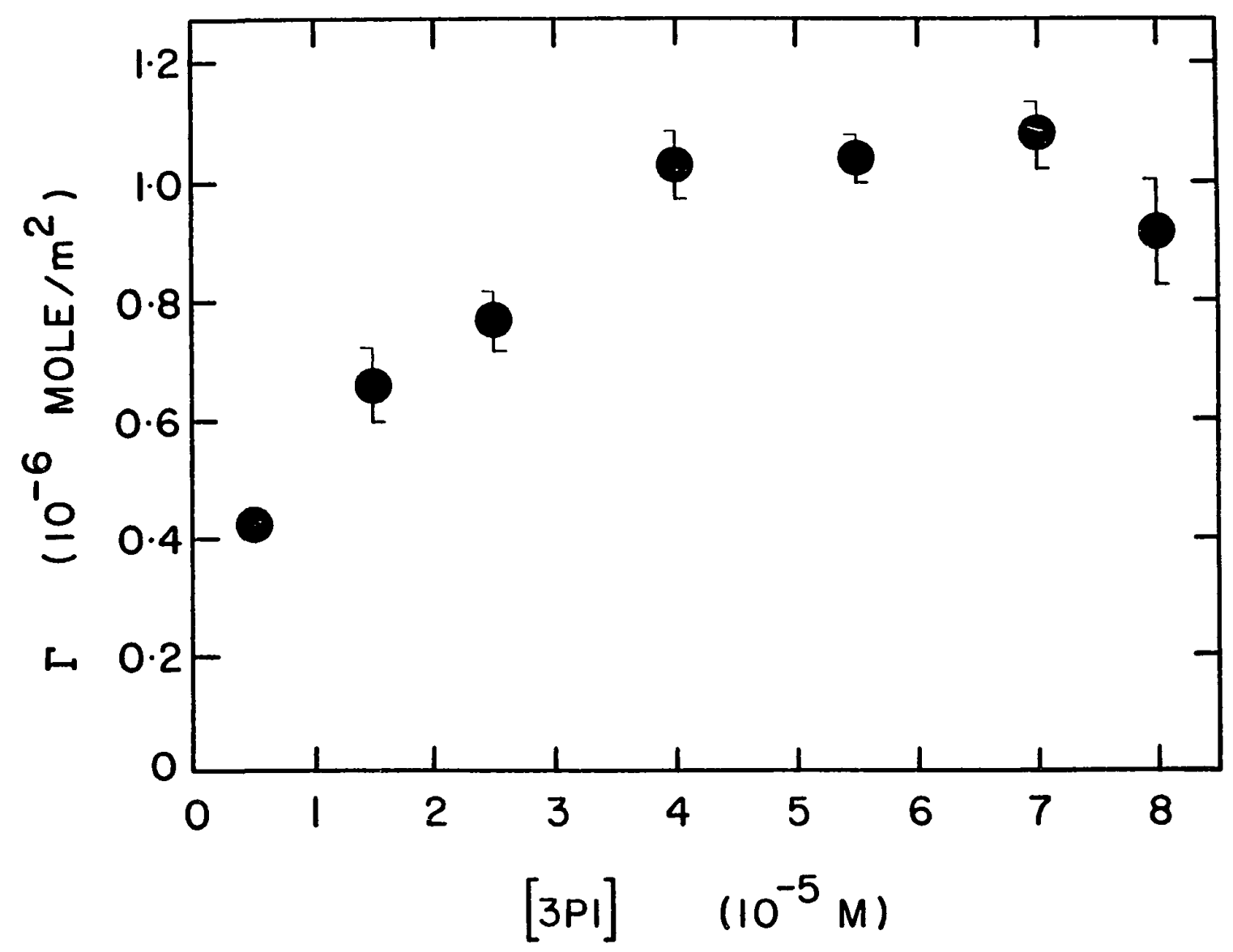

Figure 3. Adsorbed surface number density of 3-phenylindole on to phosphatidylcholine/cholesterol monolayers at $\pi=40 \mathrm{dyn} / \mathrm{cm}$ as a function of aqueous 3 -phenyl indole concentration. 


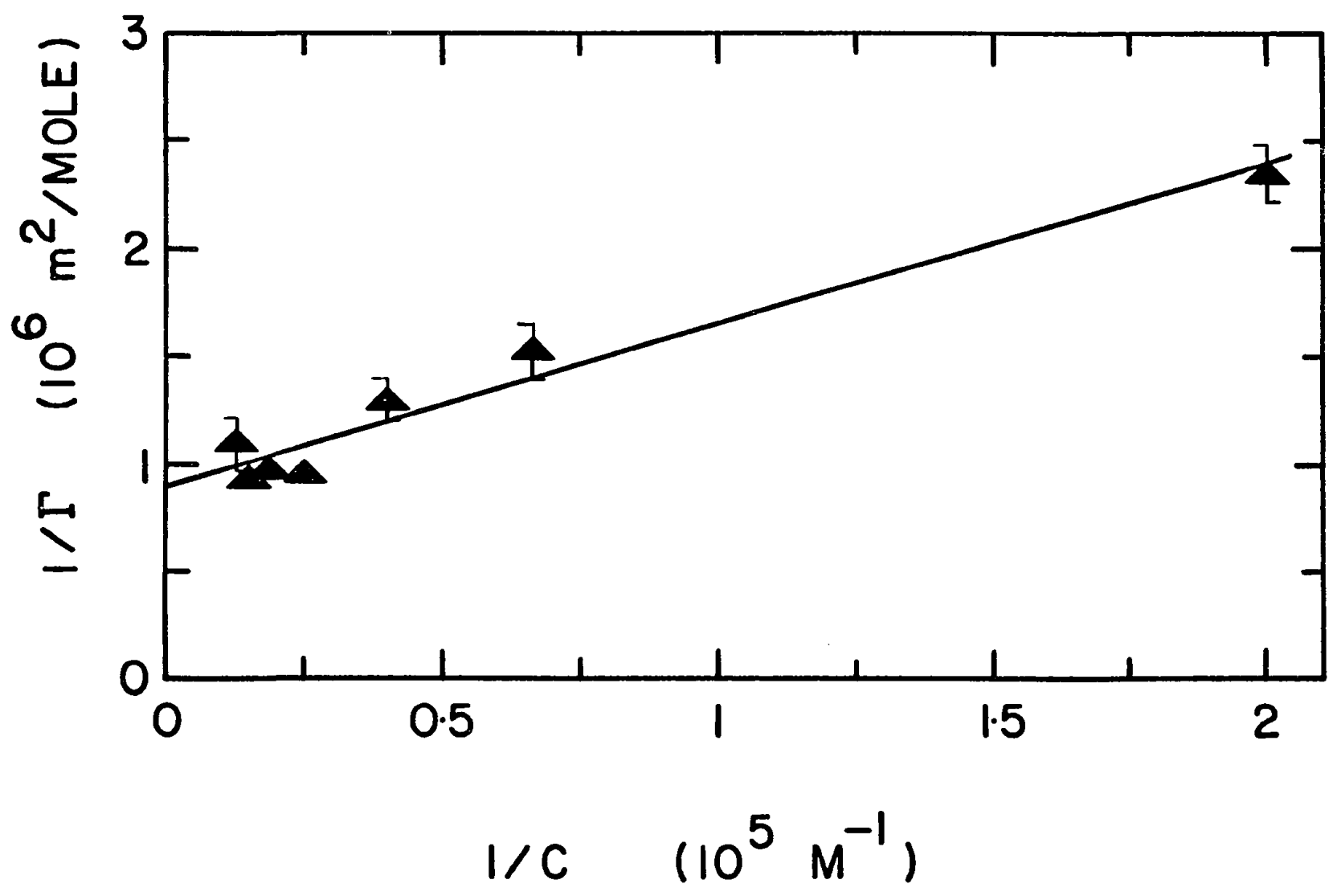

Figure 4. Experimental adsorption isotherm for 3-phenylindole. The solid line is the least-squares fit to Eq. (1). 
same data as $1 / \Gamma$ versus $1 / c$ to correspond to Langmuir adsorption. The best fit of this data to Eq. (1), shown by the solid line in Fig. 4, gives $\beta_{L}=1.35 \times 10^{-4} \mathrm{~m}$ and $\Gamma_{m}=1.11 \times 10^{-6} \mathrm{moles} / \mathrm{m}^{2}=6.68 \times 10^{-3}$ molecules $/ \AA^{2}$.

The surface potential of the subsolution in the absence of a lipid monolayer was $-330 \mathrm{mV}$ and was found to be independent of [3PI]. Fig. 5 shows representative $\Delta V-A_{2}$ curves for $P C /$ cholesterol monolayers in the presence of various concentrations of $3 P I$. The error in $\Delta V$ was $\sim 20 \mathrm{mV}$ as determined from reproducibility of the curves. The overall trend is a decrease in $\Delta V$ with increasing [3PI]. This result is further illustrated in Fig. 6 which shows $\delta(\Delta V)$ calculated by Eq. (3) with the condition of constant $\pi(40 \mathrm{dyn} / \mathrm{cm})$ and al so constant $A_{2}\left(66 \AA_{2} /\right.$ molecule), using data of the type shown in Fig. 5. Both conditions gave qualitatively the same result but the magnitude of $\delta(\Delta V)$ was greater with the condition of constant $\pi$.

Figure 7 displays the relationship between the experimental $\delta(\Delta V)$ (Fig. 6) and $\Gamma$ (Fig. 3). Error bars are the standard deviations for at least four monolayers. The best fit to Eq. (5) resulted in a slope of $-35.9 \mathrm{volt} / \mathrm{molecule} / \AA^{2}$, and this fit is shown by the solid line in Fig. 7. From this slope we obtain a value [Eq. (5)] for $\mu_{\perp}$ of $-1.7 \mathrm{D}$, where the negative sign indicates that the positive end of the 3PI dipole is toward the aqueous side of the interface. We chose $\varepsilon$ to be 3 since 3PI had such a strong influence on the $\pi-A_{2}$ curves of $P C / c h o l e s t e r o l$ and van Deenan et al. (1962) have demonstrated with synthetic phospholipids that the $\pi-A_{2}$ curve is mainly determined by the fatty acid chain while the headgroup has only a minor effect. Hence we infer that 3PI is situated with at least some portion of it near the fatty acid chains in a region 


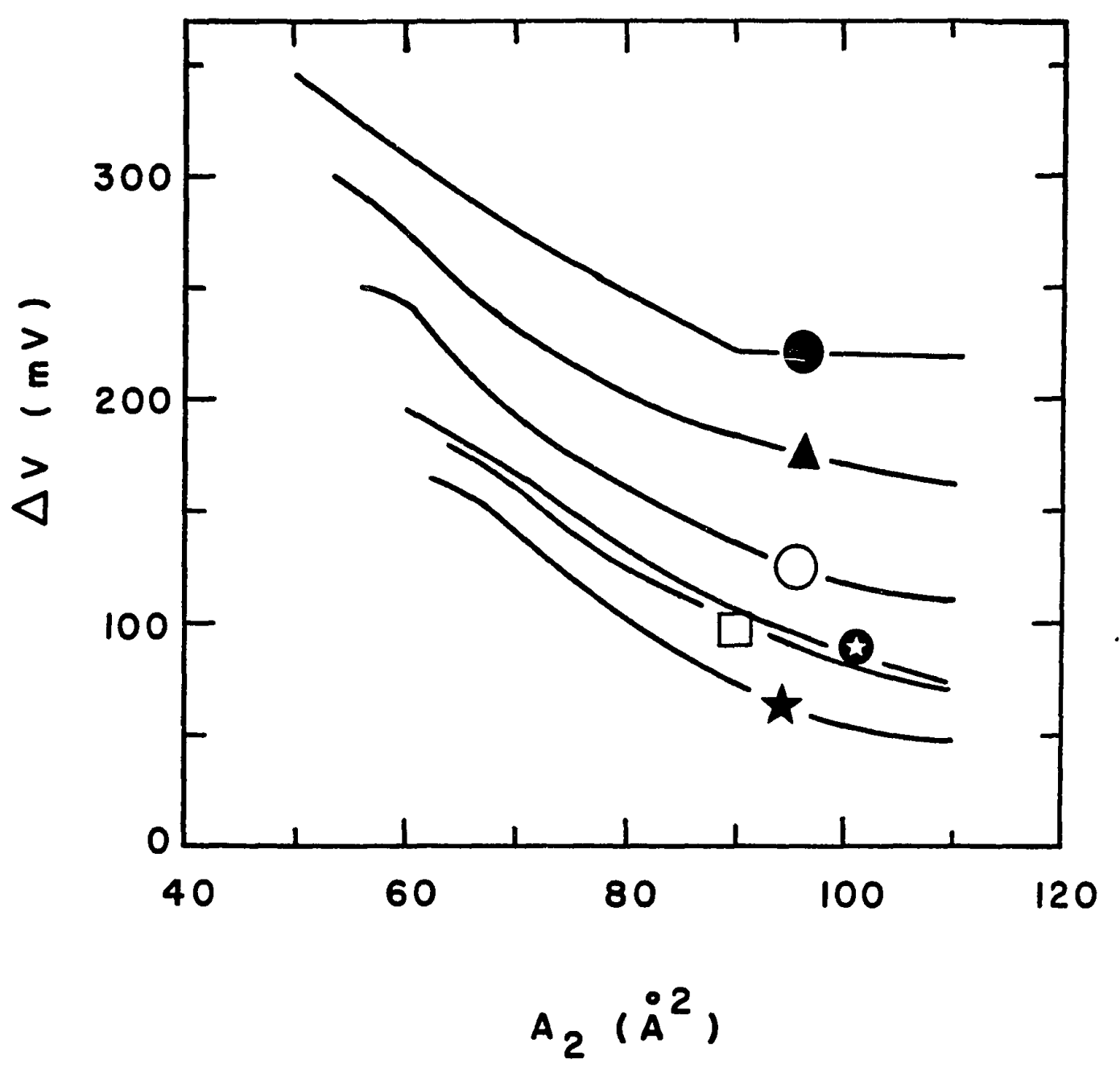

Figure 5. Surface potential of phosphatidylcholine/cholesterol monolayers as a function of area per lipid molecule. Aqueous solution $0.11 \mathrm{M} \mathrm{KCl}$ plus the indicated concentration of 3-phenylindole:

$0 \mu \mathrm{M}, \triangle 5 \mu \mathrm{M}, \bigcirc 15 \mu \mathrm{M}, \bigotimes 25 \mu \mathrm{M}, \square 40 \mu \mathrm{M}, \star 70 \mu \mathrm{M}$. 


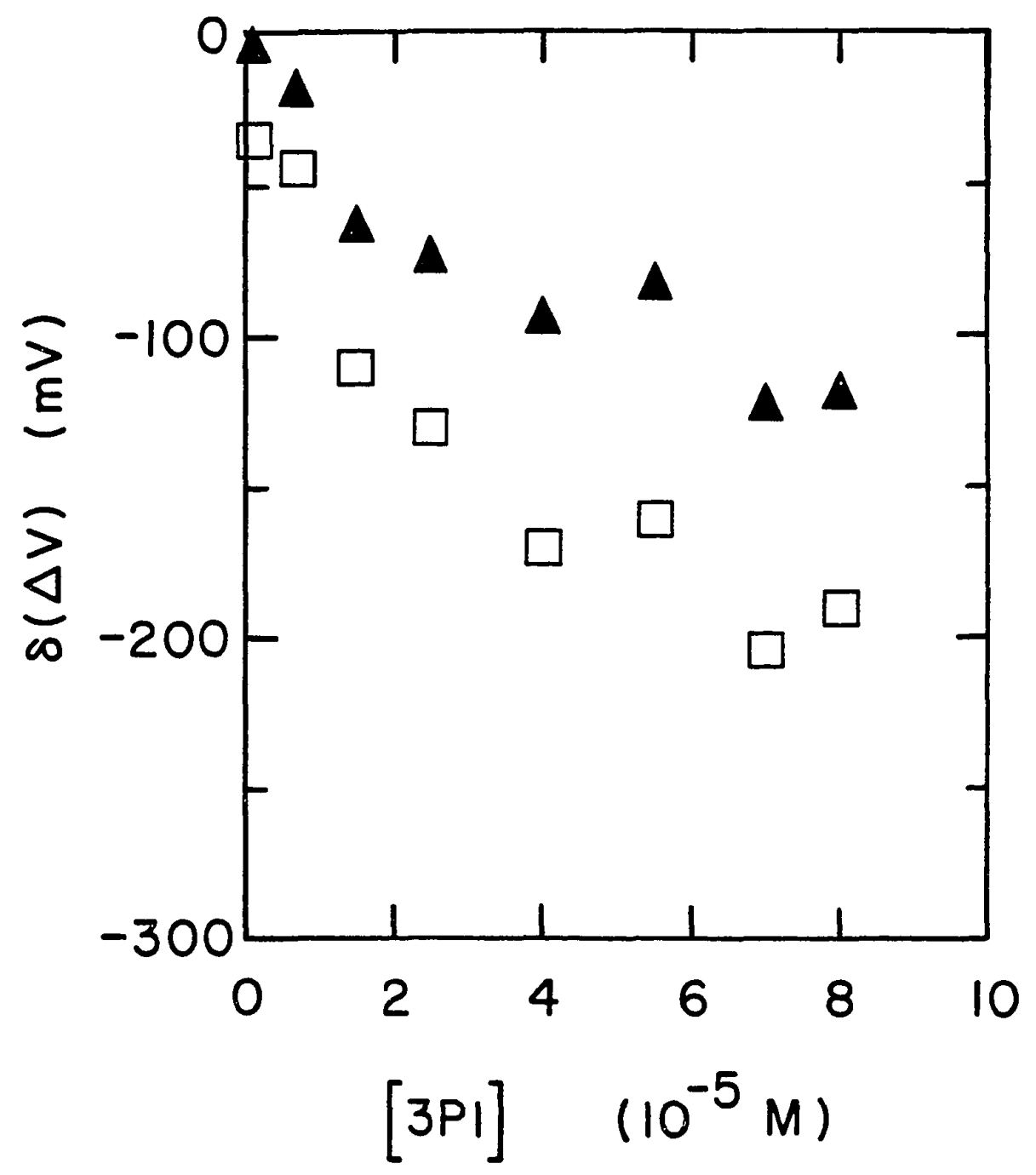

Figure 6. Change in the surface potential as a function of 3-phenylindole concentration in the aqueous solution. $\square$ at $40 \mathrm{dyn} / \mathrm{cm}$, $\Delta$ at $66 \AA^{2}$ per lipid molecule. 


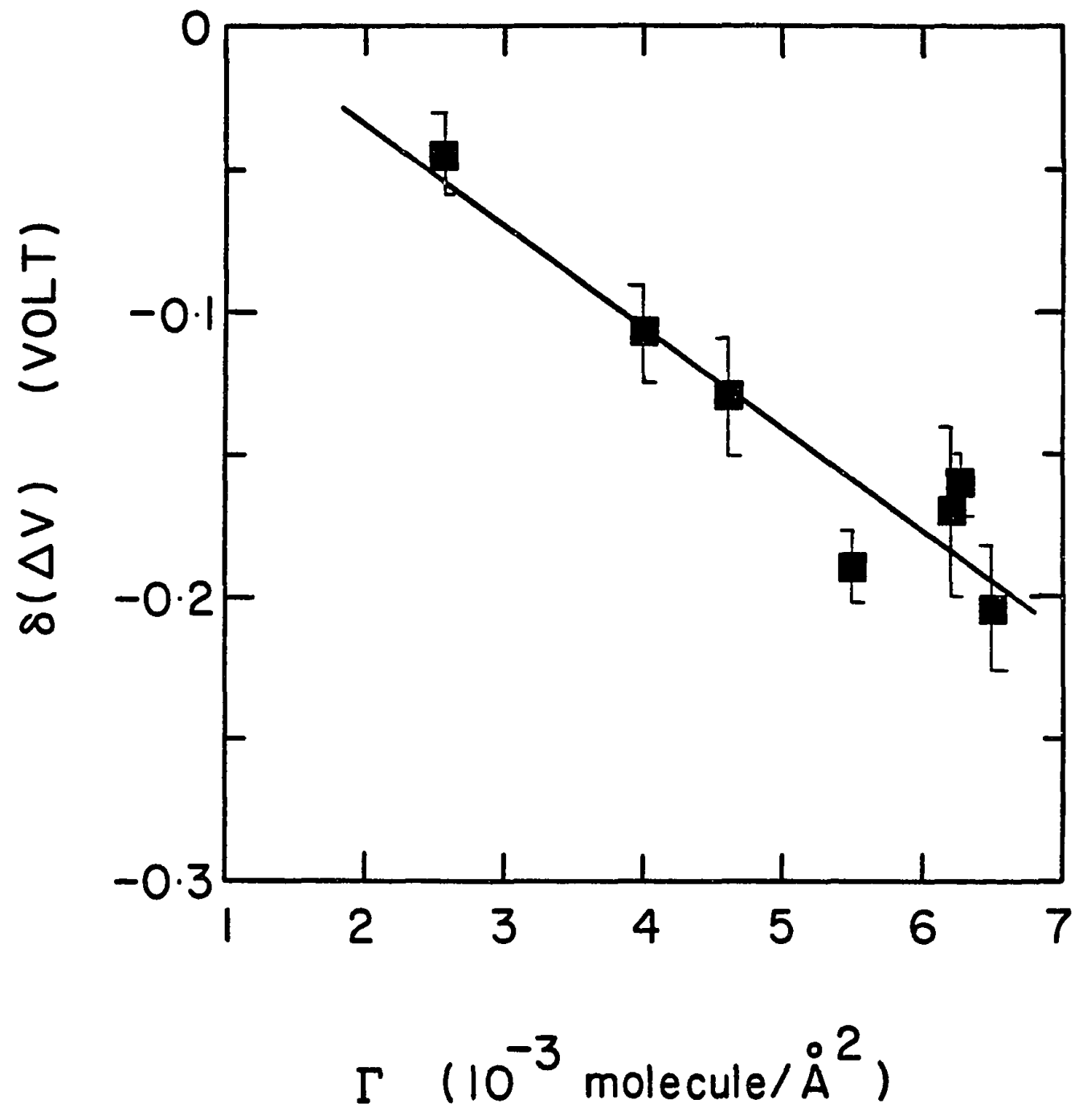

Figure 7. Change in the surface potential as a function of adsorbed surface density of 3-phenylindole. The solid line is the least squares fit to Eq. (5). 
of low dielectric constant. The surface potential in the absence of $3 P I, \Delta V_{2}$, was $+327 \mathrm{mV}$, meaning that the negative end of the lipid dipole was toward the aqueous side of the interface.

\section{DISCUSSION}

The experimental results indicate that 3PI favors partition into the lipid phase nearly as strongly as two lipophilic anions used in ion transport studies do. Using electrical conductivity measurements, Ketterer et al. (1971) determined that for tetraphenylborate $\beta=3 \times 10^{-4} \mathrm{~m}$ and for dipicrylamine $\beta=2 \times 10^{-4} \mathrm{~m}$ for adsorption onto dioleoyllecithin bilayers while we found that for 3PI, $\beta_{L}=1.35 \times 10^{-4} \mathrm{~m}$ for adsorption to $\mathrm{PC} /$ cholesterol monolayers. On the other hand they report that the maximum adsorbed number density for tetraphenylborate is $5 \times 10^{-4}$ molecules $/ \AA^{2}$ and for dipicrylamine $4 \times 10^{-4}$ molecules $/ \AA^{2}$ which are both an order of magnitude lower than the value we obtained for $3 P I\left(\Gamma_{m}=6.68 \times 10^{-3}\right.$ molecules $\left./ \AA^{2}\right)$. It is likely that electrostatic interactions are inhibiting adsorption of the two former compounds which exist as ions at the $\mathrm{pH}$ used by Ketterer et al. 3PI is in neutral form at the $\mathrm{pH}$ used in our studies. The magnitude of the adsorption is perhaps easier to visualize if the results are stated another way. Assuming the area occupied by a lipid molecule at $\pi=40 \mathrm{dyn} / \mathrm{cm}$ to be $54 \AA^{2}$ as determined from the $\pi-A_{2}$ curve for $\mathrm{PC} /$ cholesterol alone, we estimate that at the maximum adsorption density of $6.68 \times 10^{-3}$ molecule/ $/ \AA^{2}$ (or conversely, $150 \AA^{2}$ per molecule), there is one 3PI molecule for every 2.81 ipid molecules. 
The monolayer method used in this study to determine $\Gamma$ is only suitable for molecules which adsorb in such a location that the $\pi-A_{2}$ curve is affected. Even for a molecule like 3PI the method has several inherent uncertainties. There are "cavities" between the egg PC molecules in a monolayer (Shah and Schulman, 1967) and if 3PI has the right configuration to fit into the "cavities" not occupied by cholesterol, then $\Delta A_{2}$ would not be a true reflection of the number of adsorbed $3 P I$ molecules and our calculated $\Gamma$ would be an underestimate. Also, the areas were taken from the intercept with $\pi=40 \mathrm{dyn} / \mathrm{cm}$, a value which is somewhat arbitrary since there is still debate as to which thermodynamic variable should be used to determine which $\pi$ of a monolayer best mimics the condition in a (biological) bilayer (Albrecht et al., 1978; Nagle and Scott, 1978). At $\pi=40 \mathrm{dyn} / \mathrm{cm}$ the monolayer is near collapse; the lipid molecules are close together with hydrocarbon chains predominantly normal to the interface. At lower $\pi$ the 3PI molecules may have a better chance to find an adsorption site so that $I$ would be greater. Our finding that 3PI made the slope of the $\pi-A_{2}$ curve more negative, especially at larger $A_{2}$, may indicate that this is indeed occurring. Finally, since 3PI does not form a compressible monolayer, it cannot be determined by conventional techniques (Shah and Schulman, 1967; Roels and Shah, 1969) whether 3PI has a condensing or expanding influence on the monolayer. This uncertainty in $\Gamma$ could be eliminated if 3 PI could be labeled with a radioactive isotope such as ${ }^{14} \mathrm{C}$ or ${ }^{3} \mathrm{H}$ and the radioactivity from only the monolayer monitored (Muramatsu, 1973, p. 137). Then I could be measured directly and without ambiguity in a manner similar to that used for protein 
adsorption at the air/water interface (Graham and Phillips, 1979a and 1979b).

We have no evidence regarding the position of the acetone molecules which are given off as $3 P I$ sheds its solvation shell. If they stay in non-random orientation near the surface, they would make a contribution to the surface potential. However if these excess acetone molecules redissolve into the bulk aqueous solution, the effect would be constant for all 3PI concentrations, as we have assumed.

Our value for the magnitude of the normal component of the 3PI dipole moment was $1.7 \mathrm{D}$, which is slightly less than the value of $2.1 \mathrm{D}$ listed by McClellan (1974) for the total dipole moment of the parent compound indole. However, neither the orientation of the molecule at the interface nor the orientation of the dipole moment in the molecule is known with certainty. Since the value for $\mu_{\perp}$ depends on the values of $\Gamma$, all the considerations listed above for $\Gamma$ apply to $\mu_{\perp}$ as well. 


\section{CHAPTER III}

\section{ENHANCEMENT OF TETRAPHENYLARSONIUM \\ TRANSPORT BY 3-PHENYLINDOLE}

\section{MECHANISM OF LIPOPHILIC ION TRANSPORT}

The conductivity of lipid bilayer membranes increases many orders of magnitude if certain organic ions, also known as lipophilic ions, are added to the aqueous solution. Liberman and Topaly (1969) and LeBlanc (1969) investigated the increase in steady state conductivity due to several organic ions while Ketterer, Neumcke, and Läuger (1971) were able to deduce the transport mechanism by studying transient conductivity due to low concentrations $\left(\sim 10^{-7} \mathrm{M}\right)$ of the organic ion. The experimental results of Ketterer et al. (1971) were consistent with a transport scheme in which the lipophilic ions adsorb to the membrane, cross the membrane upon application of a voltage, and desorb from the opposite interface. This is shown schematically in Fig. 8. Before the voltage is applied the lipophilic ion concentration in the aqueous solution $c$ is in equilibrium with the initial ion density in the membrane $N(0)$ and the two quantities are related by the partition coefficient $\beta$ : $N^{\prime}(0)=N^{\prime}(0)=\beta C$ where a prime indicates the left side of the membrane and a double prime the right side. The rate constants for translocation from left to right and vice versa are denoted by $k^{\prime}$ and $k^{\prime \prime}$, respectively, while the rate constant for desorption is $k_{m a}$. A small value for a rate constant is equivalent to a large 


\section{AQUEOUS BILAYER AQUEOUS}

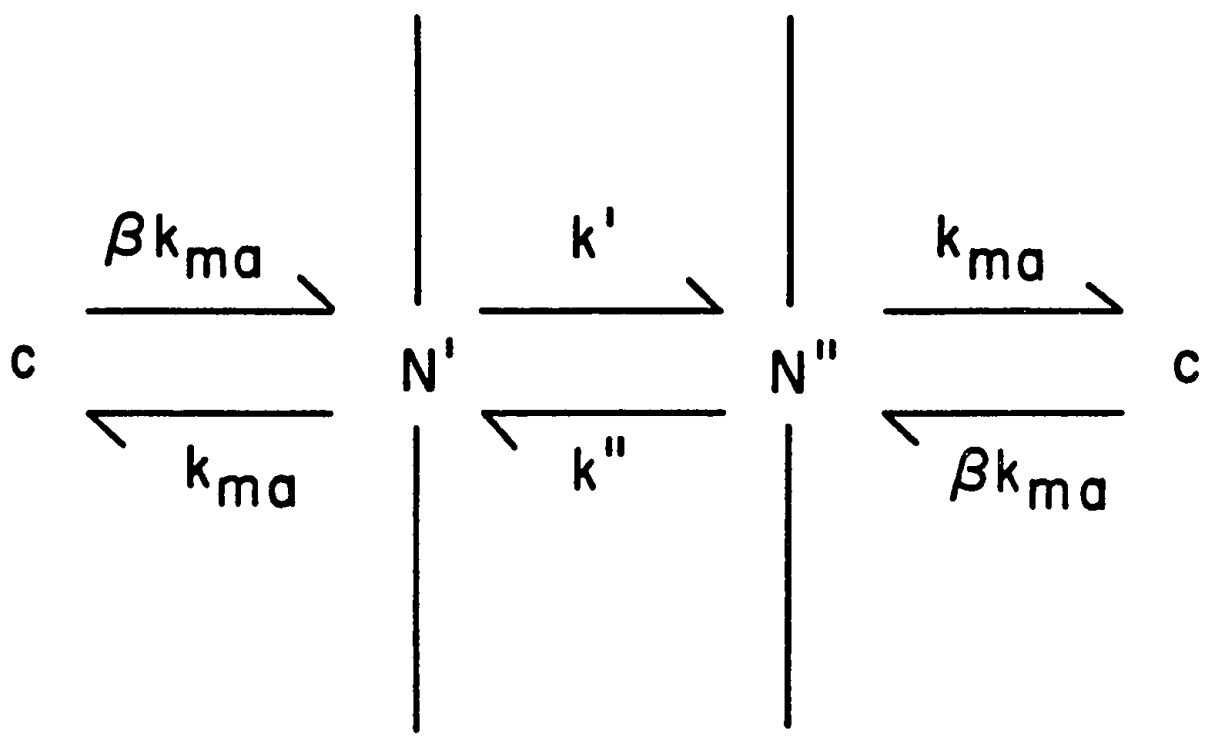

Figure 8. Kinetic model of lipophilic ion transport. 
potential energy barrier that must be overcome at that step of the transport. In deriving expressions for current and conductivity they made the usual Eyring assumption that the translocation rate constants depend on applied potential, $V$, according to

$$
\begin{aligned}
k^{\prime} & =k_{i} \exp (z u / 2), \\
k^{\prime \prime} & =k_{i} \exp (-z u / 2),
\end{aligned}
$$

where $u$ is the reduced voltage $u=F V / R T$ with the left side of the membrane at higher potential, $F$ is the Faraday, $R$ is the gas constant, $T$ is the absolute temperature, $z$ is the valency of the lipophilic ion, and $k_{j}$ is the zero voltage value of both $k^{\prime}$ and $k^{\prime \prime}$ '. The rate of change of $N^{\prime}$ and $N^{\prime \prime}$ is given by

$$
\begin{aligned}
& \frac{d N^{\prime}}{d t}=\beta k_{m a} c-k_{m a} N^{\prime}-k^{\prime} N^{\prime}+k^{\prime} N^{\prime \prime}, \\
& \frac{d N^{\prime}}{d t}=\beta k_{m a} c-k_{m a} N^{\prime}+-k^{\prime} N^{\prime}+k^{\prime} N^{\prime},
\end{aligned}
$$

After an initial ( $10 \mu \mathrm{sec}$ ) displacement current due to the charging of the lipid itself, the electrical current density, $J$, is $J=2 F\left(k^{\prime} N^{\prime}-k^{\prime} N^{\prime}\right)$. The solution of Eqs. (2) and (3) together with the voltage dependence for $k^{\prime}$ and $k^{\prime}$ ' as expressed in Eq. (1) gives the steady state current density as

$$
J=2 z F_{B c k_{i}}[\sinh (z u / 2)]\left[2 k_{i} \cosh (z u / 2) k_{m a}+1\right]^{-1} \text {. }
$$

The ratio of current density to applied voltage in the 1 imit of low voltages is called the ohmic or zero voltage conductivity $G(0)$, i.e., $G(0)=(J / V)_{V \rightarrow 0}$, and it can be shown (Ketterer et al., 1971) that the steady state zero voltage conductivity is

$$
G(0)=\left[z^{2} F^{2} B C k_{i} / R T\right]\left[2 k_{j} / k_{m a}+1\right]^{-1} .
$$


Additional information can be obtained from the manner in which the conductivity depends on the applied voltage. According to Pickar and Benz (1978) the normalized conductivity with $z=+1$ is

$$
\frac{G(V)}{G(0)}=\frac{2}{u}(1+A) \exp \left(-\omega u^{2}\right) \frac{\sinh (u / 2)}{1+A \exp \left(-\omega u^{2}\right) \cosh (u / 2)}
$$

with the kinetic limitation parameter $A$ defined as

$$
A=2 k_{i} / k_{\text {ma }} \quad \text {. }
$$

The function $\exp \left(-\omega \mathbf{u}^{2}\right)$ is a small correction factor which arises when the flux is derived from the generalized Nernst-Planck equation using an image-force potential energy barrier as an approximation for the actual potential energy barrier across the membrane (Andersen and Fuchs, 1975; Appendix III). For a simple Eyring approximation [Eq. (1)] the shape of the barrier is neglected and $\omega=0$. The relationship between the shape of the energy barrier and a modified voltage dependence of $k^{\prime}$ and $k^{\prime \prime}$ is discussed by Benz et al. (1975; Appendix A).

In a comparative study of oppositely charged lipophilic ions, Pickar and Benz (1978) found that for the positively charged ion tetraphenylarsonium, $\left(\mathrm{TPhA}^{+}\right)$, whose chemical formula is shown in Fig. 9, the normalized conductivity fit the case of $A \approx 0$ indicating that $k_{m a} \gg k_{i}$. This means that ions can be supplied to and from the membrane as fast as they are translocated across the central energy barrier. For $\mathrm{TPhA}^{+}$then, the steady state zero voltage conductivity given by Eq. (5) reduces to

$$
G(0)=F^{2} \beta_{C k_{i}} / R T .
$$




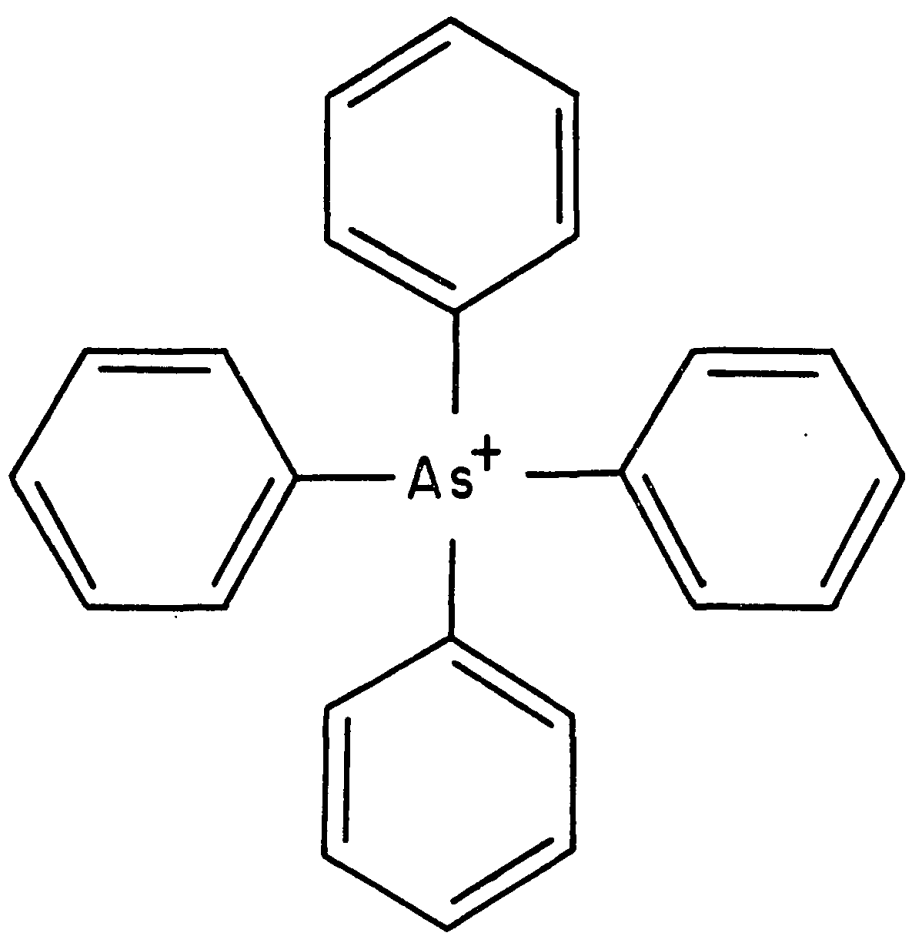

Figure 9. Chemical formula of tetraphenylarsonium. 
Following the argument of Haydon and Hladky (1972, p. 257), the zero voltage conductivity depends on the mobility of the ion $\bar{u}$ in the membrane and the concentration $c_{m}$ of the ion in the membrane: $G(0) \propto \bar{u}_{m}$. The concentration of ions in the membrane is related to the concentration of lipophilic ions in the aqueous phase by a Boltzmann factor. [The thermal energy distribution of the ions determines the amount of partition into the membrane and thermal energy is a function of velocity of the ions. For a derivation of the Boltzmann factor for velocity distribution see, for example, Anderson (1971).] If 3PI decreases the dipole potential at the lipid/aqueous interface by $\Delta \psi$ but leaves $\bar{u}$ unchanged, then the conductivity in the presence of $3 P I, G(0)$, is expected to be related to the conductivity in the absence of $3 P I, G *(0)$, by a Boltzmann factor containing the change in dipole potential:

$$
G(0)=G *(0) \exp (-z F \Delta \psi / R T)
$$

\section{MATERIALS AND METHODS}

Bilayer lipid membranes were formed at room temperature by the brush technique of Mueller et al. (1963) across the aperture $(1.7 \mathrm{~mm}$ diameter) in a Tefion cup set in an acrylic container; the membrane was bathed by symmetrical aqueous solutions. Silver/silver chloride electrodes (surface area $\sim 1.1 \mathrm{~cm}^{2}$ ) were made from silver foil and wire (Montana Assay, Portland, OR) and chloridized in $0.1 \mathrm{~N} \mathrm{HCl}$. When $\mathrm{TPhA}^{+}$was present in the aqueous solution, the membrane usually became black within four minutes but since the current sometimes increased with time, measurements were taken after a period of about 40 minutes 
to allow for equilibration. The circuit used for steady-state DC measurements is shown in Fig. 10. A steady voltage was applied to the membrane and the current measured with a picoammeter (Keithley 480). Current I was measured at voltages $V$ ranging from 25 to 200 $\mathrm{mV}$ or until the membrane broke. The conductivity $G[G=I /(V \cdot A)$ where $A$ is the geometrical area of the aperture] extrapolated by parabolic curve fit to zero voltage, $G(0)$, is the value used in comparisons to determine the effect of the pesticide.

The membrane forming solution contained a mixture of phosphatidylcholine and cholesterol in decane. Cholesterol mole fraction was $Y=.22$ and the total 1 ipid content was $12 \mathrm{mg} / \mathrm{ml}$. Egg phosphatidylcholine (PC) from Sigma Chemical Co., St. Louis, MO, showed a single spot by thin layer chromatography and was used without further purification. Recrystallized, lyophilized cholesterol was a gift from Dr. D. McClure of the Chemistry Department while n-decane (Aldrich Gold Label 99+\%) was obtained from Aldrich Chemical Co., Milwaukee, WI. 3-phenylindole (3PI) was synthesized by the method of Fischer and Schmidt (1888) as described in Chapter II. In these experiments the aqueous solution consisted of $0.1 \mathrm{M} \mathrm{KCl}$ and a buffer $\left(B^{-3}\right)$ of potassium phosphate:potassium citrate:boric acid 0.002M:0.002M:0.0005M. The desired amount of 3PI was added from a stock solution in acetone by injecting it below the surface of the solution during continuous stirring. The $\mathrm{pH}$ was adjusted to 6.9 with $\mathrm{HCl}$. For the studies on tetraphenylarsonium transport, tetraphenylarsonium chloride hydrate from Aldrich was used to make an aqueous stock solution which also contained the $\mathrm{KCl}$ and the buffer. The final concentration of $\mathrm{TPhA}^{+}$ 


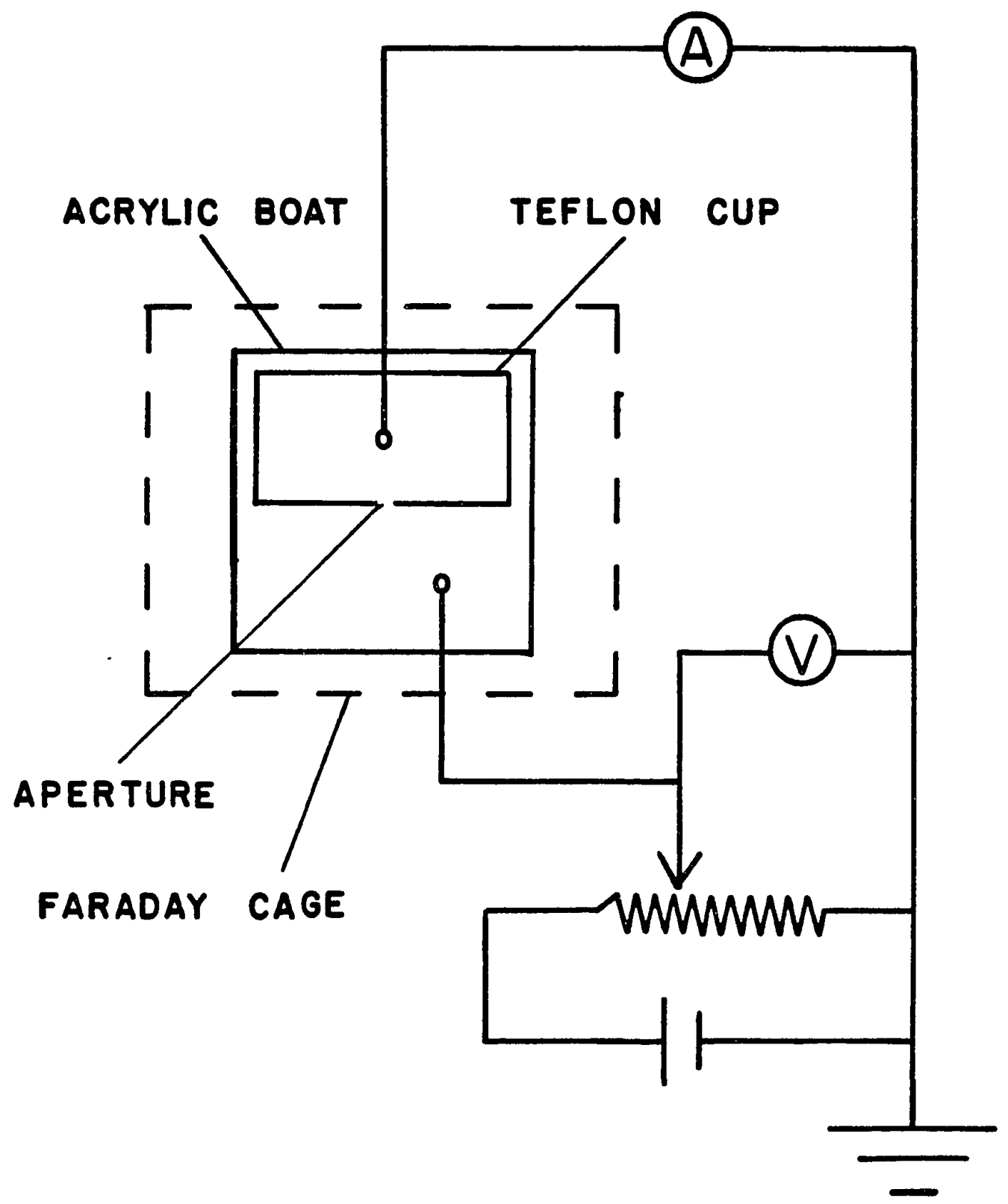

Figure 10. Schematic diagram of experimental set-up for measuring $\overline{\mathrm{DC} \text { conductivity. }}$ 
was $5 \times 10^{-3} \mathrm{M}$. Reagent grade inorganic chemicals and distilled, deionized water from a Millipore Q2 system, Millipore Corp., Bedford, MA, were used for all solutions.

When prepared in the manner just described, solutions containing more than $45 \mu \mathrm{M} 3 \mathrm{PI}$ together with $5 \times 10^{-3} \mathrm{M} \mathrm{TPhA}^{+}, 0.1 \mathrm{M} \mathrm{KCl}$ and $B^{-3}$ were noticeably opaque; the opacity was reversed by addition of excess water but not by addition of more acetone. The experimental concentration was restricted to $[3 P I] \leq 45 \mu M$ in which range a true solution appeared to exist. In an effort to determine the cause of the precipitation phenomenon, some further investigations were performed and it was determined that three components had to be present to cause the opacity. Thus, solutions containing $5 \times 10^{-3} \mathrm{M} \mathrm{TPhA}^{+}$and $80 \mu \mathrm{M} 3 \mathrm{PI}$ were clear; solutions containing $5 \times 10^{-3} \mathrm{M} \mathrm{TPhA}^{+}$and up to $1 \mathrm{M} \mathrm{KCl}$ were clear. A solution of $80 \mu \mathrm{M} 3 \mathrm{PI}$ was salted out by $1 \mathrm{M} \mathrm{KCl}$, appearing as particulates in the bulk and as a pronounced layer of flecks on the surface, but this appearance was quite different from the uniform opacity mentioned above.

Cleanliness of the Teflon cup was ensured by routinely boiling it in ethanolic $\mathrm{NaOH}$ on the day of the experiment. To enhance stability of the membranes, the aperture of the cup was painted with the lipid solution and dried thoroughly under $\mathrm{N}_{2}$ prior to the experiment. 
RESULTS AND DISCUSSION

The electrical conductivity of a $\mathrm{PC} /$ cholesterol membrane at a cholesterol mole fraction of 0.22 was sma11, approximately $3 \times 10^{-8}$ $\mathrm{S} / \mathrm{cm}^{2}$. No change in this conductivity was detected when membranes were formed in the presence of up to $70 \mu \mathrm{M} 3 \mathrm{PI}$ in the aqueous solution or when the membrane forming solution contained 3PI:1 ipid at mole ratios up to 0.6 . Our finding that $3 P I$ does not affect lipid bilayer conductivity suggests that 3PI does not directly 1 iberate free fatty acids or disrupt the normal bilayer structure. From this result we conclude that 3PI acts as neither a carrier nor a pore in these conditions. Electron microscopic studies would help to determine whether 3PI causes a noticeable disruption of the structure of liposomes (Barratt and Weaver, 1979). A further test of disruption would be the measurement of single channel currents.

The effect of $3 P I$ on transport of the lipophilic cation $\mathrm{TPhA}^{+}$ is illustrated by the data in Fig. 11. This figure shows the zero voltage conductivity of $\mathrm{PC} /$ cholesterol bilayers as a function of $3 \mathrm{PI}$ concentration when $5 \times 10^{-3} \mathrm{MTPhA}^{+}$was in the aqueous solution; the error bars are the standard deviation for at least four members. The conductivity increased with increasing [3PI]: at $45 \mu \mathrm{M} 3 \mathrm{PI}$ the conductivity was about 900 times the value it was in the absence of 3PI. The experimental $G(0)$ were used in Eq. (8) to calculate values of the product $B k_{i}$ which are displayed in Table $I$. Also in this table are the values of parameter $A$ as determined by the best fit of the experimental $G(V) / G(0)$ versus voltage to Eq. (6). A value of $\omega=0$ gave a better fit than a non-zero $\omega$ and was used at all 3PI concentrations. 


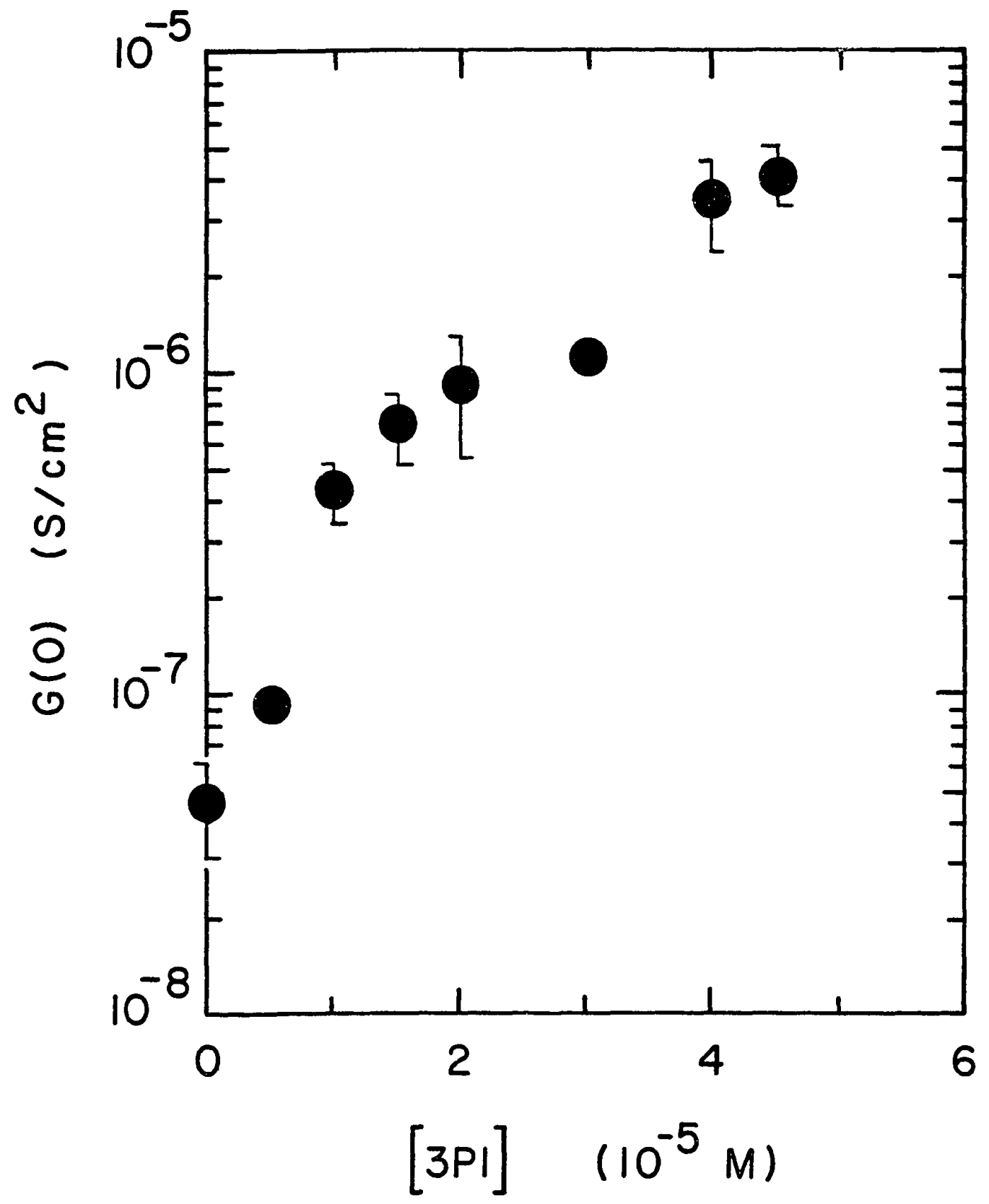

Figure 11. Tetraphenylarsonium zero voltage conductivity as a function of 3-phenylindole concentration. 
TABLE I

KINETIC PARAMETERS OF TETRAPHENYLARSONIUM TRANSPORT IN THE PRESENCE OF 3PI

\begin{tabular}{clc}
\hline $\begin{array}{c}\text { 3PI concentration } \\
\left(10^{-5} \mathrm{M}\right)\end{array}$ & $\begin{array}{c}\beta K_{i} \\
\left(10^{-7} \mathrm{~cm} / \mathrm{s}\right)\end{array}$ & $A$ \\
\hline 0.0 & 0.023 & $-.004 \pm .012$ \\
0.5 & 0.050 & $-.005 \pm .011$ \\
1.0 & 0.22 & $-.005 \pm .007$ \\
1.5 & 0.35 & $+.004 \pm .010$ \\
2.0 & 0.47 & $-.006 \pm .007$ \\
3.0 & 0.55 & $+.015 \pm .012$ \\
4.0 & 1.8 & $-.003 \pm .010$ \\
4.5 & 2.1 & $+.012 \pm .013$
\end{tabular}

The value of $0.023 \times 10^{-7} \mathrm{~cm} / \mathrm{s}$ for $B k_{j}$ in the absence of $3 P I$ is consistent with the value given by Pickar and Benz (1978) for dioleoyllecithin-decane membranes $\left(0.063 \times 10^{-7} \mathrm{~cm} / \mathrm{s}\right)$ since cholesterol would decrease the conductivity due to positively charged TPhA ${ }^{+}$ (Szabo, 1974) and hence decrease the product $\beta k_{i}$. As Pickar and Benz point out, it is not possible to get the individual values of $\beta$ and $k_{j}$ for TPhA ${ }^{+}$and consequently it is not possible to say conclusively whether the increased conductivity in the presence of $3 \mathrm{PI}$ is due to increased adsorption of $\mathrm{TPhA}^{+}$or to faster translocation across the membrane.

We found that the kinetic limitation parameter $A$ defined in Eq. (7) was essentially zero within experimental error at all 3PI concentrations. From this we conclude that 3PI does not measurably change the ratio of the rate constants and that the translocation step still occurs much more slowly than the adsorption and desorption steps. 
Fig. 12 shows the potential change calculated according to Eq. (9) using the data of Fig. 11, and also for comparison the monolayer potential changes at $\pi=40 \mathrm{dyn} / \mathrm{cm}$ shown previously in Fig. 6 . The potentials calculated by the two methods qualitatively agree but $\delta(\Delta V)$ is typically more negative by $40-50 \mathrm{mV}$. This is consistent with both experimental and theoretical observations of other investiyators. In a tabulation of the experimental results of himself and others, Hladky (1979) found that monolayer surface potentials are consistently of larger magnitude than potentials calculated by Eq. (9). Andersen et al. (1978) present theoretical arguments relating the macroscopic surface potential normally measured in monolayer experiments and the micropotential $\Phi$ which would be sensed by an individual ion, e.g. $\mathrm{TPhA}^{+}$. The macropotential is due to the average of a smeared layer of charge whereas the micropotential takes into account the fact that the layer is actually composed of discrete charges. Using a model based on a lattice of discrete adsorbed charges, they conclude that $\Phi$ will, in general, be lower in magnitude than the macropotential. Similar arguments probably hold true for potentials due to discrete dipoles. As a final point, note that the monolayer $\delta(\Delta V)$ with a condition of constant area per lipid molecule (see Fig. 6) is in much better agreement with the $\mathrm{TPhA}^{+}$results by Eq. (9), but the agreement is probably fortuitous since there is no reason why the area per lipid molecule should remain constant in a bilayer as 3PI is added whereas there is reason to believe the surface tension may be constant under constraint of the Plateau border. 


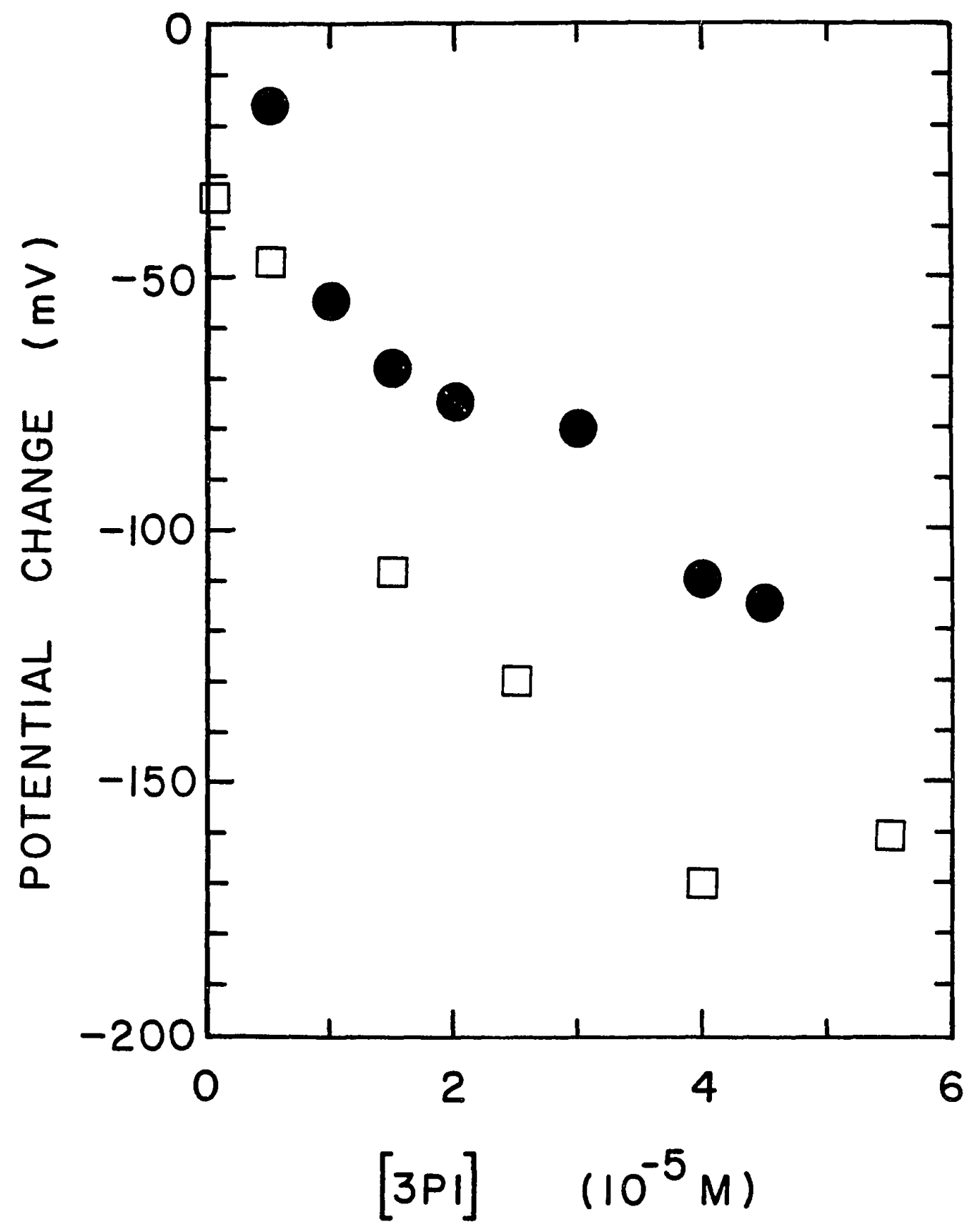

Figure 12. Potential change as a function of 3-phenylindole concentration. bilayer conductivity with tetraphenylarsonium according to Eq. (8), $\square$ monolayer $\delta(\Delta V)$ at $\pi=40 \mathrm{dyn} / \mathrm{cm}$. 
There are other factors besides the electrostatic change in dipole potential which may contribute to the change in $\mathrm{TPhA}^{+}$conductivity in the presence of $3 \mathrm{PI}$, such as an increased viscosity or a decrease in thickness of the bilayer. These are discussed in more detail in Chapter IV in connection with lipophilic anion transport. Another point to consider is that if $3 P I$ is influencing the solvation of $\mathrm{TPhA}^{+}$as we might infer from the precipitation phenomena described in the Materials and Methods section of this chapter, then perhaps $B$ is being greatly increased by the presence of $3 P I$ and $\mathrm{KCl}$. This would be a chemically selective phenomenon as distinct from an increase in $\beta$ for any positive ion due to an electrostatic lowering of the potential energy barrier at the interface. In a comparative study of the interaction of tetraphenylarsonium and tetraphenylborate with various solvents, Coetzee and Sharpe (1971) state that $\mathrm{TPhA}^{+}$may undergo specific interaction with water and that the orientation of solvent molecules by $\mathrm{TPhA}^{+}$is different from that by tetraphenylborate. 


\section{CHAPTER IV}

\section{EFFECT OF 3-PHENYLINDOLE ON LIPOPHILIC ANION TRANSPORT}

\section{MECHANISM OF TRANSIENT LIPOPHILIC ION TRANSPORT}

The partition coefficient $\beta$ for lipophilic anions from water to lipid bilayers is on the order of $10^{4}$ times the partition coefficient for lipophilic cations (Liberman and Topaly, 1969), presumably because the dipole moment of phospholipid is oriented with the positive end toward the interior of the bilayer (Hladky, 1972). The origin of this dipole moment was discussed in Chapter I. The chemical formulas of the lipophilic anions tetraphenylborate (TPhB ${ }^{-}$) and dipicrylamine $\left(\mathrm{OPA}^{-}\right)$are given in Fig. 13. In the absence of an applied potential these ions are located in deep potential energy minima close to the aqueous/membrane interface on each side of the membrane (Ketterer et al., 1971). When a voltage is applied across the membrane a current results which is due to some of the ions traversing the membrane from one potential energy minimum to the other. In contrast to $\mathrm{TPhA}^{+}$, the lipophilic anions have rate constants (refer to Fig. 8) such that $k_{\text {ma }} \ll k_{j}$ where $k_{i}$ is the zero voltage value of both $k^{\prime}$ and $k^{\prime \prime}$, so that on a short time scale the total number of ions in the membrane is conserved: $N^{\prime}+N^{\prime \prime}=2 N_{\text {ads }}$ where $N_{\text {ads }}$ is the number of lipophilic ions adsorbed per unit area to one boundary region of the membrane. The fact that $\beta$ is large for anions means that the current is initially large but the fact that $k_{\text {ma }} \ll k_{j}$ means 

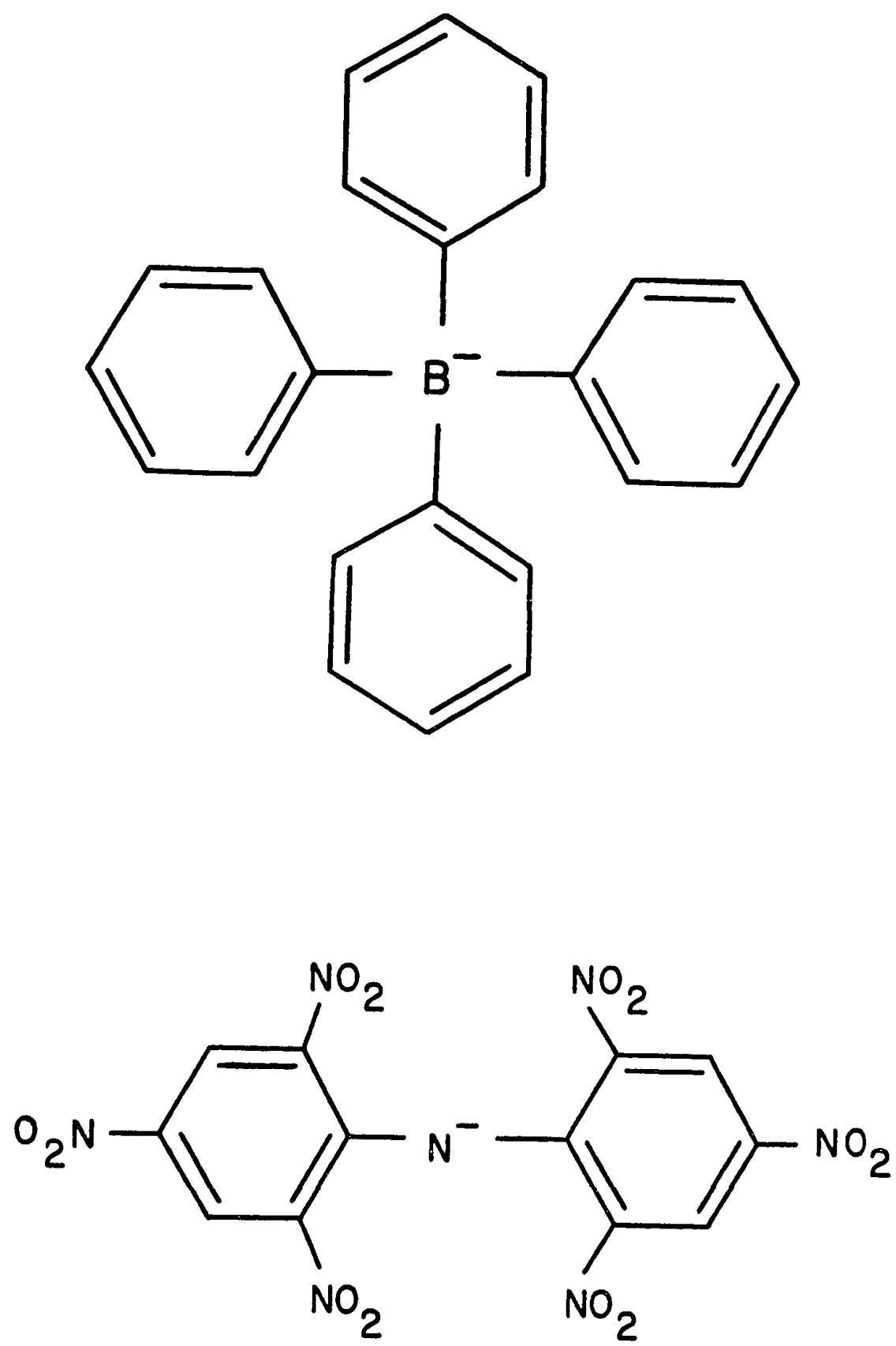

Figure 13. Chemical formulas of tetraphenylborate (upper) and dipicrylamine (lower). 
that the current quickly drops to near zero. The steady state current due to $\mathrm{TPhA}^{+}$as discussed in Chapter III is due to transport across the membrane while for the lipophilic anions only the transient current is related to membrane processes. [The steady state current for lipophilic anions is of the order of magnitude predicted by a diffusion limitation in the aqueous phase (Ketterer et al., 1971) but Andersen and Fuchs (1975) found inconsistencies in this explanation.]

Referring again to Fig. 8, the rate of change of $N^{\prime}$ and $N^{\prime \prime}$ when $k_{\text {ma }} \ll k_{i}$ is given by

$$
\begin{aligned}
& \frac{d N^{\prime}}{d t}=-k^{\prime} N^{\prime}+k^{\prime} N^{\prime \prime}, \\
& \frac{d N^{\prime}}{d t}=k^{\prime} N^{\prime}-k^{\prime} N^{\prime \prime},
\end{aligned}
$$

The translocation rate constants depend on applied voltage according to the usual Eyring assumption

$$
\begin{aligned}
k^{\prime} & =k_{j} \exp (u / 2), \\
k^{\prime \prime} & =k_{j} \exp (-u / 2),
\end{aligned}
$$

where $u$ is the reduced voltage defined in Chapter III. The solution of Eqs. (1), (2), and (3) together with the initial condition that $N^{\prime}(0)=N^{\prime}(0)=N_{\text {ads }}$ yields the number densities as a function of time:

$$
\begin{aligned}
N^{\prime}(t) & =N_{\text {ads }}\left\{\frac{\exp (-u / 2)}{\cosh (u / 2)}+\frac{\tanh (u / 2)}{\exp \left[2 k_{i} \cosh (u / 2) t\right]}\right\}, \\
N^{\prime}(t) & =N_{\text {ads }}\left\{\frac{\exp (u / 2)}{\cosh (u / 2)}-\frac{\tanh (u / 2)}{\exp \left[2 k_{j} \cosh (u / 2) t\right]}\right\} .
\end{aligned}
$$

When voltage is still being applied but current is no longer flowing across the membrane, the ions have been redistributed and the number of ions which have crossed the membrane is 
$\Delta N=\left[N^{\prime}(\infty)-N^{\prime}(\infty)\right] / 2=N_{\text {ads }} \tanh (u / 2)$. Andersen and Fuchs (1975)

found that their data for tetraphenylborate was fit better by

$$
\Delta N=N_{\text {ads }} \tanh (\mathrm{bu} / 2)
$$

where $b$ is presumed to represent the fraction of applied voltage effective in moving ions through the membrane. Equation (5) corresponds to the situation that as the applied voltage increases the number of lipophilic ions with sufficient energy to cross the barrier will increase and at large voltages the number of ions crossing will approach the number of ions adsorbed to one interface, $\mathrm{N}_{\text {ads }} \cdot$ The number of adsorbed ions is related to the concentration of ions in the bulk aqueous phase, $c$, through the partition coefficient $B$ by $\mathrm{N}_{\text {ads }}=B C$.

The electrical current density $J=z F\left(k^{\prime} N^{\prime}-k^{\prime} N^{\prime}\right)$; substituting from Eqs. (3) and (4), the current density becomes

$$
J(t)=2 z \mathrm{FN}_{\mathrm{ads}} k_{i} \sinh (u / 2) \exp (-t / \tau),
$$

where

$$
\tau=\left[2 k_{j} \cosh (u / 2)\right]^{-1}
$$

We see from Eq. (6) that in the absence of any modifiers 1ipophilic anion transport results in a transient current which decays exponentially with time, $I(t)=I_{0} \exp (-t / \tau)$, where $I_{0}$ is the current at time zero. $I_{0}$ is not directly measurable because in the first few usec after the application of a voltage pulse the lipid itself gives rise to a displacement current (capacitive spike). Hence experimentally $I_{0}$ is found by extrapolation from the remainder of the exponential decay. 
The conductivity $G$ is defined as $G=I /(V \cdot A)$ where $A$ is the area of the aperture so the initial conductivity at a given applied voltage is $G_{0}=I_{0} /(V \cdot A)$. The experimental results will be given in terms of the ohmic limit of the time constant $\tau(0)=[\tau(V)]_{V \rightarrow 0}$ and of the initial conductivity $G_{0}(0)=\left[G_{0}(V)\right]_{V \rightarrow 0^{*}}$. Equations $(6)$ and $(7)$ can be used to show that the zero voltage values are related to the partition coefficient and the rate constant for translocation by

$$
G_{0}(0)=z^{2} F^{2} B C k_{i} / R T,
$$

and

$$
\tau(0)=\left(2 k_{j}\right)^{-1},
$$

where the substitution $N_{\text {ads }}=\beta C$ has been made.

\section{POTENTIAL ENERGY BARRIERS FOR LIPOPHILIC IONS}

Transport of lipophitic ions can be described as a passage of the ion across activation energy barriers. Following Eyring rate theory as presented by Ketterer et al. (1971), the rate constant of a transport process is equal to a frequency factor $f$ times the exponential of the free energy $\Delta F$ of activation. Thus when there is no applied voltage

$$
\begin{aligned}
k_{i} & =f_{i} \exp \left(-\Delta F_{i} / R T\right), \\
k_{m a} & =f \exp (-\Delta F / R T), \\
\beta k_{m a} & =f_{a} T_{a} \exp \left(-\Delta F_{a} / R T\right),
\end{aligned}
$$

where $l_{a}$ is the jump length for a jump from the aqueous solution into the potential energy minimum near the interface. The frequency factors $f$ and $f_{a}$ are both of the order of $R T / h N_{A} \sim 6 \times 10^{12} \mathrm{sec}^{-1}$ where $h$ is 
Planck's constant and $N_{A}$ is Avogadro's number (Ketterer et al., 1971). The barrier encountered by a lipophilic ion in crossing from the aqueous phase through the membrane to the aqueous phase on the opposite side is shown schematically in Fig. 14. The origin of this intrinsic barrier was discussed in the second section of Chapter I. The presence of an externally applied potential will distort the shape of the potential energy barrier.

Now it remains to show how the activation barriers are related to the experimentally observable quantities $\tau(0), G_{0}(0)$ and $Q_{a d s}=e N_{a d s}$ where $e$ is the electronic charge. The partitioning of the ion is determined by the rate of adsorption minus the rate of desorption so if we let $\Delta F_{p}=\left(\Delta F-\Delta F_{a}\right)$ then combining Eqs. (11) and (12) we get $Q_{a d s}=$ $\left(\right.$ ect $\left.\mathrm{T}_{\mathrm{a}}\right) \exp \left(\Delta \mathrm{F}_{\mathrm{p}} / \mathrm{RT}\right)$. The time constant is related to $\Delta \mathrm{F}_{i}$ through Eqs. (9) and $(10), \tau(0)=\left(1 / 2 f_{i}\right) \exp \left(\Delta F_{j} / R T\right)$. The zero voltage conductivity depends on both $\beta$ and $k_{j}$ as seen in Eq. (8). To simplify notation (refer to Fig. 14) let $\Delta F_{g}=\Delta F_{j}-\Delta F_{p}$, then

$$
G_{0}(0)=\left[z^{2} F^{2} c f_{i} 1_{a} / R T\right]\left[\exp \left(-\Delta F_{g} / R T\right)\right] .
$$

\section{SEPARATION OF ELECTROSTATIC AND NON-ELECTROSTATIC EFFECTS ON ION TRANSPORT}

If all free energies are expressed in units of RT, then the zero voltage conductivity is proportional to the exponential of $\left(-\Delta F_{j}\right)$ and to the exponential of $\left(\Delta F_{p}\right)$, and the height of either one or both of these energy barriers could be changed by the presence of an additional dipole located near the membrane/aqueous interface. A special case is that of the adsorption plane of the lipophilic ions being located outside (on the aqueous side) of the dipole layer. Then $\Delta F_{j}$ 


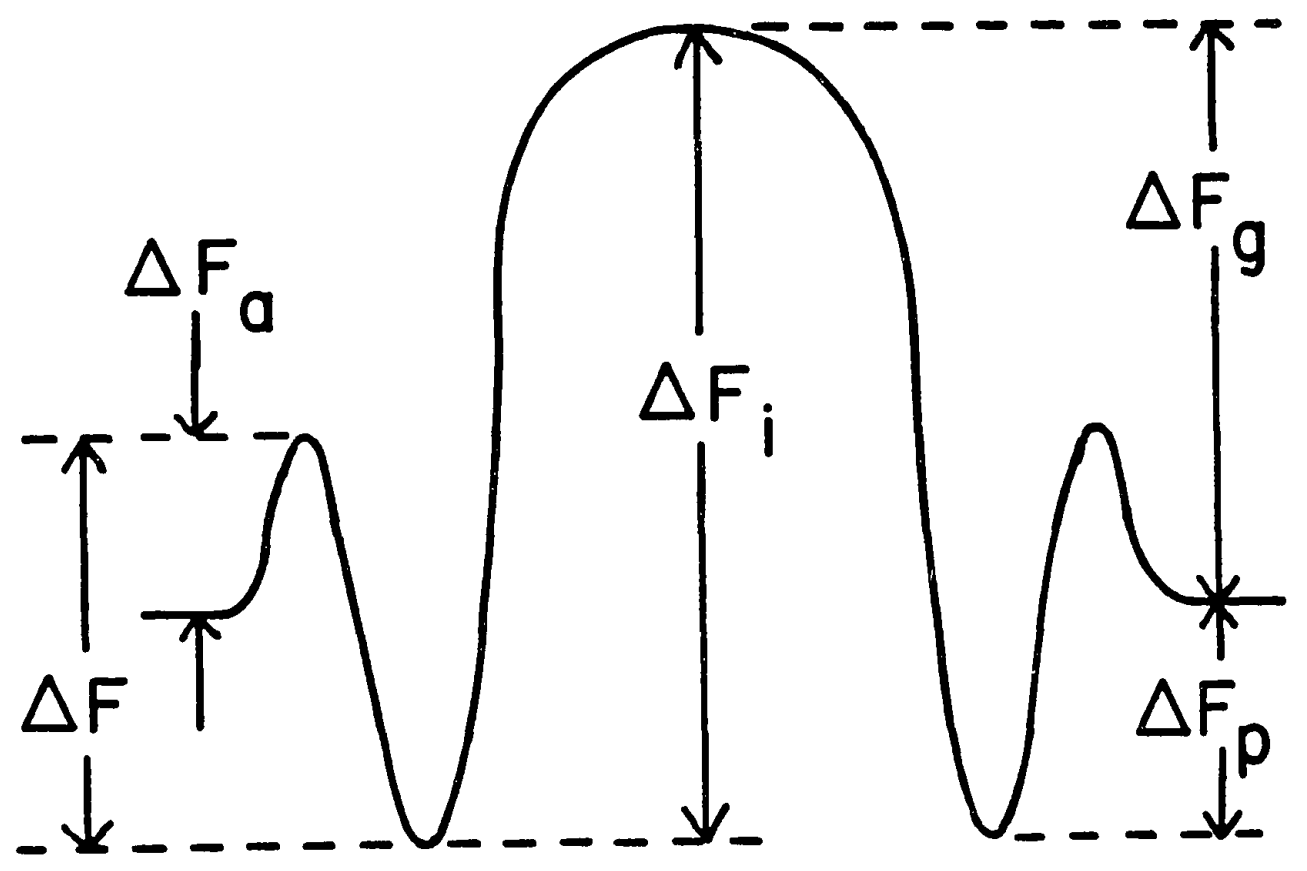

Figure 14. Activation energies involved in ion transport through the membrane. Adapted from Ketterer, Neumcke, and Läuger (1971). 
(translocation) will be affected but $\Delta F_{p}$ (partition) will be unaffected (Benz and Cros, 1978). However in general we can say that an additional dipole at the interface will influence $\Delta F_{g}$ and the zero voltage conductivity will vary as the exponential of the change in $\left(-\Delta F_{g}\right)$. Let $\Delta V_{d}$ be the change in the electric potential of the middle of the bilayer with respect to the aqueous phase due to the presence of $3 \mathrm{PI}$. Then with the condition that 3PI affects only the electric potential but leaves all other parameters in Eq. (13) unchanged, the relative conductivity $\bar{G}$ will be

$$
\bar{G}=\frac{G_{0}(0)}{G_{0}^{*}(0)}=\exp \left(-z F \Delta V_{d} / R T\right),
$$

where $G_{0}(0)$ is the zero voltage conductivity in the presence of $3 P I$ and $G_{0}^{*}(0)$ in its absence. $R, T$, and $F$ have their usual thermodynamic meanings while $z$ is the valency of the lipophilic ion. By comparing the conductivities of oppositely charged lipophilic ions (Szabo, 1976; Pickar and Benz, 1978) we can determine the magnitude of the dipole potential change caused by the presence of 3PI since

$$
\exp \left(-F \Delta V_{d} / R T\right)=\left[\overline{G^{+}} / \overline{G^{-}}\right]^{\frac{1}{2}},
$$

where a superscript + denotes the positively charged lipophilic ion $(z=+1)$ and a superscript - denotes the negatively charged lipophilic ion $(z=-1)$.

Non-electrostatic influences on zero voltage conductivity are implicit in Eq. (13) but the dependence of the current on non-electrostatic factors is perhaps better seen when Nernst-Planck electrodiffusion formalism is used to describe the ion transport. The current 
density is given by

$$
J=-z \bar{u}(x)\left[R T \frac{d c_{m}}{d x}+z c_{m}(x, t) F \frac{d V}{d x}-c_{m}(x, t) \frac{d W}{d x}\right],
$$

where $x$ is the coordinate normal to the plane of the bilayer, $\bar{u}(x)$ is the mobility of lipophilic ions in the membrane phase, $c_{m}(x, t)$ is the concentration of lipophitic ions in the membrane phase, $V$ is the externally applied potential, and $W(x)$ is the inherent potential energy barrier within the membrane shown graphically in Fig. 14 (Goldman, 1943; Andersen and Fuchs, 1975). In order to solve this equation it is necessary to make certain assumptions regarding how $\bar{u}, c_{m}, v$, and $w$ depend on distance $x$. If, as a first approximation, it is assumed that $\bar{u}$ is independent of $x$, then the current density and the conductivity vary linearly with mobility. When Eq. (16) is integrated with respect to $x$, the thickness of the membrane appears in the limits of integration. If, as we assumed before, 3PI changes the electric potential in the middle of the bilayer by an amount $\Delta V_{d}$ and, in addition, has some non-electrostatic effect, then the relative zero voltage conductivity $\bar{G}$ will be

$$
\bar{G}=\frac{G_{0}(0)}{G_{0}^{*}(0)}=f(\bar{u}) \exp \left(-z F \Delta V_{d} / R T\right),
$$

where $G_{0}(0)$ and $G_{0}^{*}(0)$ have the same meaning as in $E q .(14)$. The function $f(\bar{u})$ describes the change in ion mobility when 3PI is present as well as effects of thickness changes which modify the shape of the potential energy barrier. It is possible to determine the non-electrostatic contribution to $G_{0}(0)$ by a comparison of the conductivities of 
oppositely charged lipophilic ions (Szabo, 1976; Pickar and Benz, 1978) since by Eq. (17)

$$
f(\bar{u})=\left[\overline{G^{+}} \cdot \overline{G^{-}}\right]^{\frac{1}{2}},
$$

where the + and - superscripts have the same significance as in Eq. (15).

Szabo (1976) showed that the enhancement of Tipophilic anion conductivity and the suppression of lipophilic cation conductivity by increasing proportions of cholesterol in monoolein bilayers could be attributed almost entirely to a change in dipole potential. Andersen et al. (1976) found that phloretin, an inhibitor of hexose and chloride transport in red blood cells, dramatically increases cation conductance and decreases anion conductance of membranes treated with ion carriers or with lipophilic ions and concluded that these changes are due primarily to a change in dipole potential, with a smaller contribution from fluidity changes. The modification of ion transport by the widely used herbicide 2,4-dichlorophenoxyacetic acid (2,4-D) was studied by Smejtek and Paulis-Illangasekare (1979a, 1979b), who propose that 2,4-D also acts by a dipole mechanism and, furthermore, that the changes affect mostly the translocation step of ion transport for the carrier nonactin and for the lipophilic ions TPhA ${ }^{+}$ and $\mathrm{TPhB}^{-}$.

\section{MATERIALS AND METHODS}

Bilayer lipid membranes were formed by the same technique and using the same sources as described in Chapter III. The total lipid concentration was usually $12 \mathrm{mg} / \mathrm{ml}$ but a more dilute solution was 
necessary in the absence of 3PI so that the membrane would thin within a reasonable time. Sodium tetraphenylborate, reagent grade from Mallinckrodt, St. Louis, MO, was stored as an ethanol solution while $2,2^{\prime}, 4^{\prime}, 4^{\prime}, 6,6^{\prime}$-hexanitrodiphenylamine (more commonly called dipicrylamine and referred to hereafter as $\mathrm{DPA}^{-}$) from Aldrich was prepared as a stock solution in $10^{-2} \mathrm{M} \mathrm{NaOH}$. The aqueous solution, prepared on the day of the experiment, contained $0.1 \mathrm{M} \mathrm{NaCl}$, a buffer $\left(B^{-3}\right)$ of sodium phosphate: sodium citrate:boric acid 0.002M:0.002M:0.0005M, plus either $10^{-7} \mathrm{M} \mathrm{TPhB}^{-}$or $10^{-8} \mathrm{M} \mathrm{DPA}^{-}$. The lipophilic ion concentrations chosen were low enough that saturation effects (Ketterer et a]., 1971; Wang and Bruner, 1978) were avoided. The desired amount of 3PI was added from a stock solution in acetone by injecting it below the surface of the solution during continuous stirring. The $\mathrm{pH}$ was adjusted to 6.9 with $\mathrm{HCl}$.

The electrical measurements were performed using a two-electrode voltage clamp. Measurements were usually taken 15 minutes after the membrane turned black or, for membranes which thinned very slowly, as soon as it was completely black. As shown schematically in Fig. 15, a voltage pulse was applied across the membrane by a fast-settling D/A converter (DAC80, Burr-Brown, Tucson, $A Z$ ) with the voltage output taken across a resistive load. Most of the experiments were done with a cholesterol mole fraction of 0.22 for which the membrane had a specific capacitance of $0.39 \mu \mathrm{F} / \mathrm{cm}^{2}$ (Hanai, Haydon and Taylor, 1965). Membrane current was converted to a voltage by a differential amplifier (LH0062, National Semiconductor, Santa Clara, CA) in a virtual ground configuration. A transient recorder (Biomation Model 802, Palo Alto, 


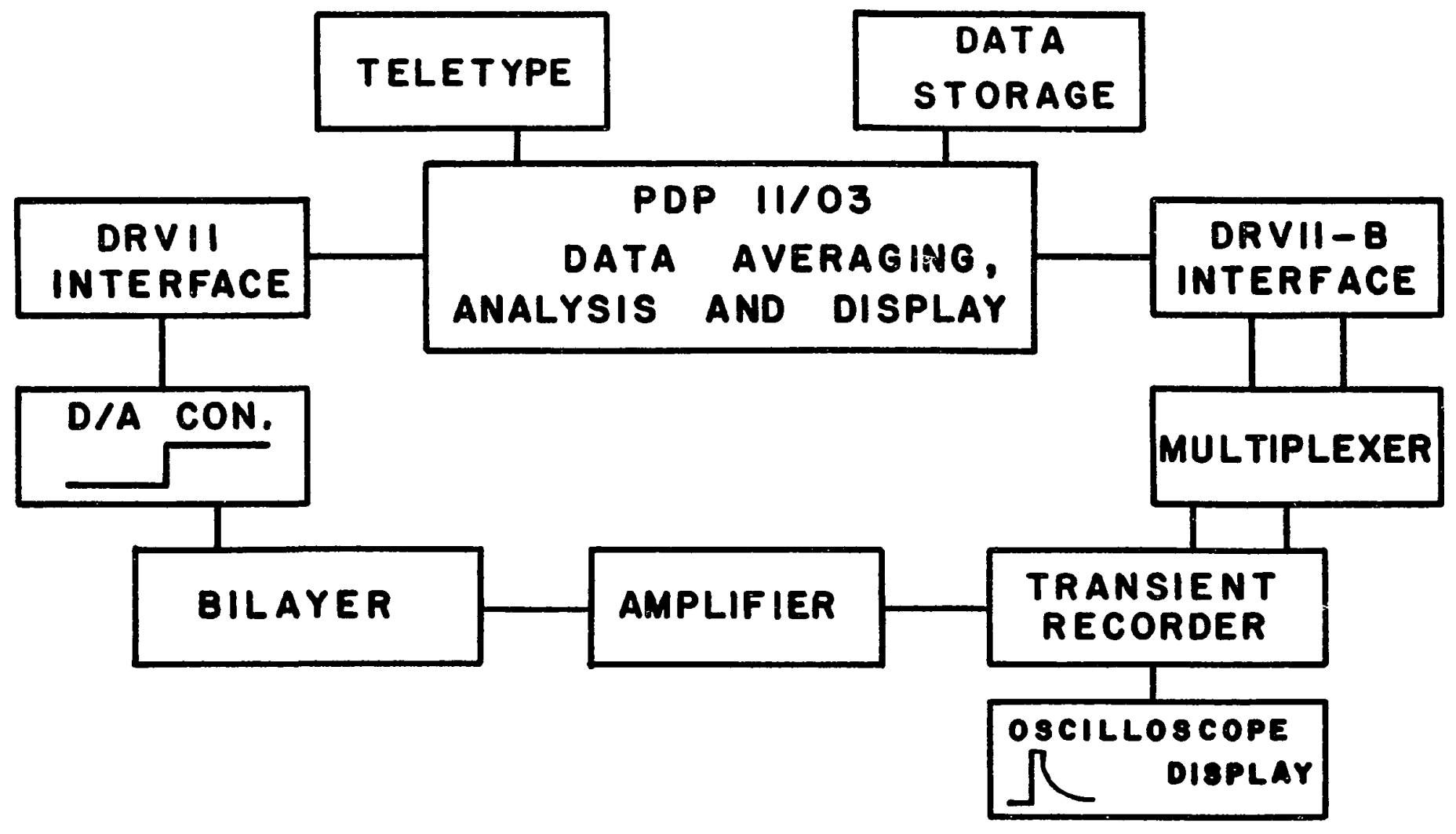

Figure 15. Block diagram of experimental set-up for measuring transient currents. 
CA) transformed the analog voltages to digital form and stored the information in its 1000 word memory in which each word consisted of 8 bits. A muliplexer permitted automatic reading of the settings of the transient recorder and transfer of the digital data by burst mode from the transient recorder through the DRV11-B direct memory access interface to the PDP 11/03. The voltage pulse amplitude, pulse duration and number of pulses were controlled by PDP 11/03 software (Laboratory Support Library or LSILIB) using the DRV11 interface.

The average of a number of pulses was displayed graphically on the computer terminal as current versus time and natural logarithm of current versus time and then curve fit to an exponential decay. In this way the time constant $\tau$ and the current extrapolated to zero time $I_{0}$ were obtained and the transferred charge density $\Delta Q$ $\left[e \Delta N=\Delta Q=\left(I_{0} \cdot \tau\right) / A\right.$ where $A$ is the geometrical area of the aperture $]$ was computed. The experimental results were printed out on a teletype and aiso stored on a floppy disk, allowing for further analysis. For comparisons with the results of experiments with other ions, the zero voltage conductivity $G_{0}(0)$ was determined from a parabolic curve fit of the low voltage $(\leq 125 \mathrm{mV})$ initial conductivities $G_{0}\left[G_{0}=I_{0} /(V \cdot A)\right]$. The zero voltage time constant $\tau(0)$ was also determined from a parabolic curve fit extrapolating $\tau$ from low voltages ( $\leq 125 \mathrm{mV}$ ) to zero voltage. Our values of $G_{0}(0)$ and $\tau$ in the absence of 3PI were consistent with those found by Andersen and Fuchs (1975) for $\mathrm{TPhB}^{-}$and by Ketterer et al. (1971) for both $\mathrm{TPhB}^{-}$and $\mathrm{DPA}^{-}$, after taking into account the different lipids used. 
RESULTS

The dependence of the zero voltage conductivity on 3PI concentration is shown in Fig. 16 for both $\mathrm{TPhB}^{-}$and $\mathrm{DPA}^{-}$; error bars are the standard deviation for 4 - 7 membranes. The most notable feature of these data is the small magnitude of the change in $G_{0}(0)$ for both $\mathrm{TPhB}^{-}$and $\mathrm{DPA}^{-}$in the presence of $3 \mathrm{PI}, \mathrm{G}_{0}(0)$ for $\mathrm{DPA}^{-}$was $5.2 \times 10^{-4}$ $\mathrm{S} / \mathrm{cm}^{2}$ in the absence of $3 \mathrm{PI}$ and decreased with increasing [3PI], attaining a value of $0.71 \times 10^{-4} \mathrm{~s} / \mathrm{cm}^{2}$ at $6.0 \times 10^{-5} \mathrm{M} 3 \mathrm{PI}$. When the lipophilic ion was $\mathrm{TPhB}^{-}, \mathrm{G}_{0}(0)$ was $4.6 \times 10^{-5} \mathrm{~S} / \mathrm{cm}^{2}$ in the absence of $3 P I$ and decreased with increasing [3PI], having a value of $3.8 \times 10^{-5} \mathrm{~s} / \mathrm{cm}^{2}$ at $8.0 \times 10^{-5} \mathrm{M} 3 \mathrm{PI}$. Hence $\mathrm{G}_{0}(0)$ decreased less than an order of magnitude for both lipophilic anions in the presence of 3PI.

In Fig. 17 the zero voltage time constant is plotted as a function of 3PI concentration for the two lipophilic anions $\mathrm{TPhB}^{-}$and $\mathrm{DPA}^{-}$; error bars are the standard deviations for $4-7$ membranes. Note that again the magnitude of the change due to 3PI is smal1. $\tau(0)$ for $D A^{-}$transport was $3.7 \times 10^{-4} \mathrm{sec}$ in the absence of $3 \mathrm{PI}$ and increased with increasing [3PI], reaching a value of $13 \times 10^{-4} \mathrm{sec}$ at $6.0 \times 10^{-5}$ $M$ 3PI. The time constant for $[3 P I]>2 \times 10^{-5} M$ was nearly independent of the aqueous 3PI concentration. $\tau(0)$ for $\mathrm{TPhB}^{-}$transport decreased slightly with increasing [3PI], a trend opposite to that exhibited by $\tau(0)$ for $D P A^{-}$. In the absence of $3 P I, \tau(0)$ for $T_{P h}^{-}$was $3.4 \times 10^{-2}$ sec while at the maximum concentration of $8.0 \times 10^{-5} \mathrm{M} 3 \mathrm{PI}, \tau(0)$ was $2.05 \times 10^{-2} \mathrm{sec}$. According to the model of 1 ipophilic ion transport outlined earlier, this implies by Eq. (8) that for $\mathrm{DPA}^{-}$transport $k_{j}$ 


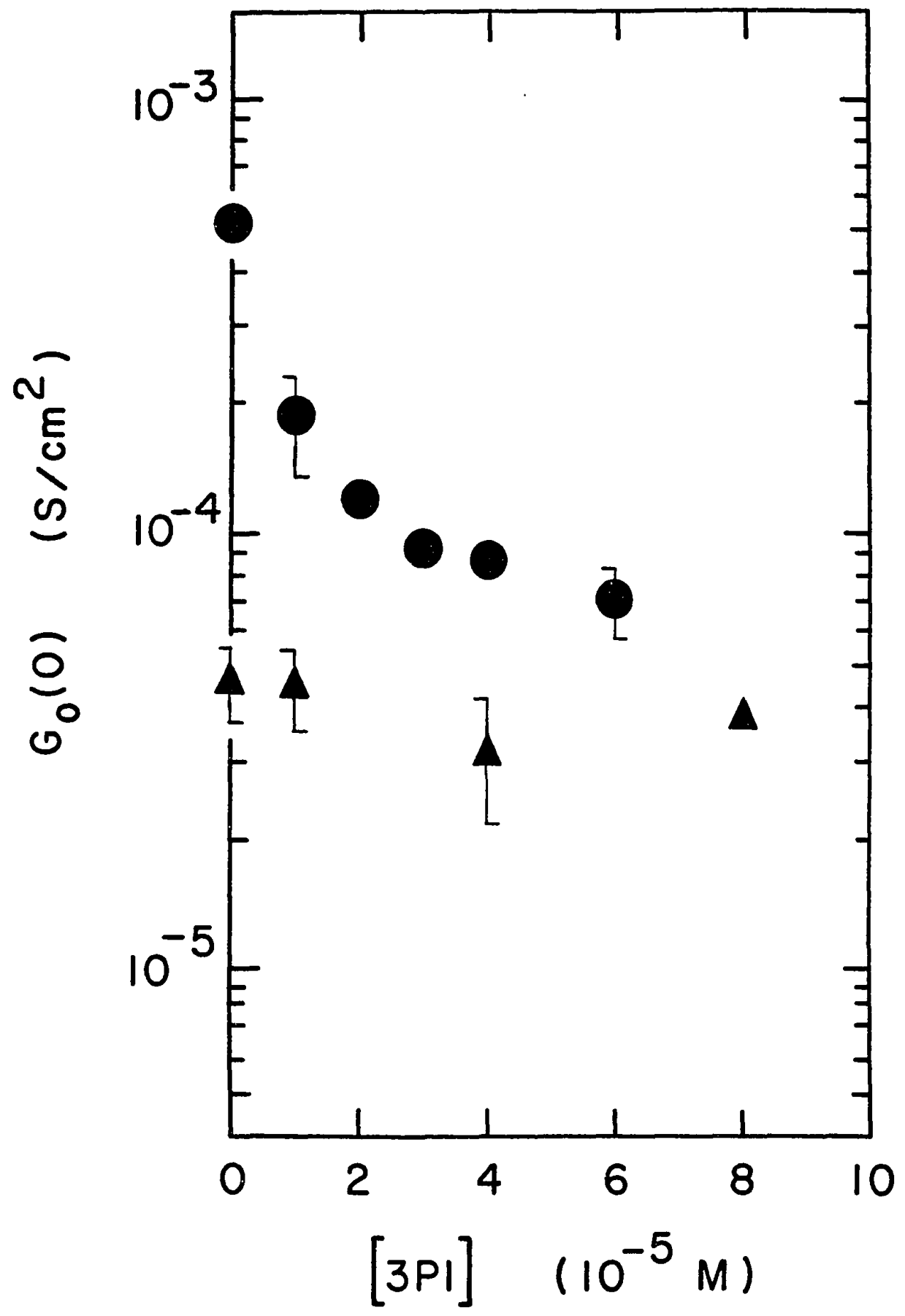

Figure 16. Lipophilic anion zero voltage initial conductivity as a function of 3-phenylindole concentration: $\triangle \mathrm{DPA}^{-}, \triangle \mathrm{TPhB}^{-}$. 


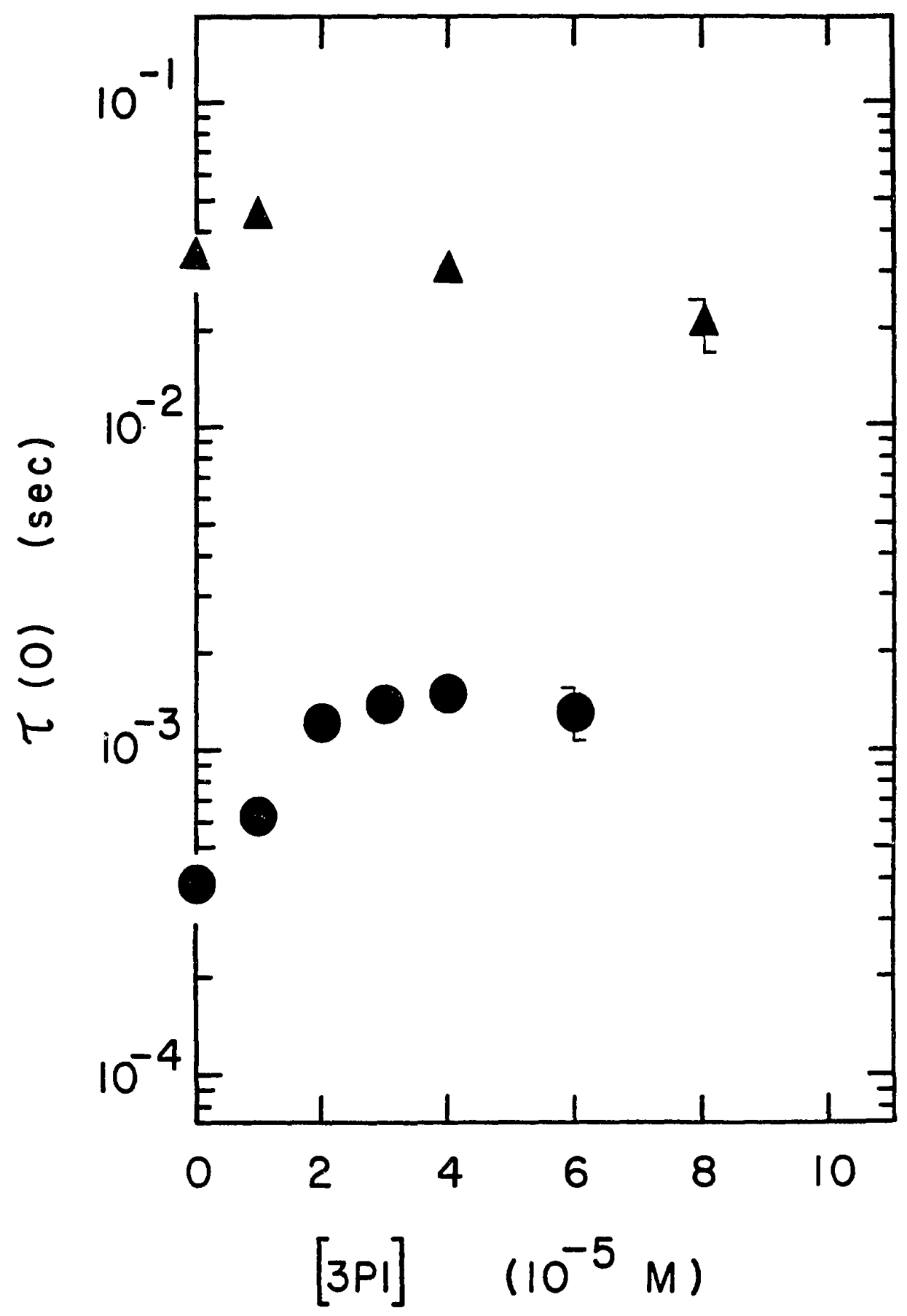

Figure 17. Zero voltage time constant for lipophilic anion transport as a function of 3-phenylindole concentration: $\triangle \mathrm{DPA}^{-}, \triangle \mathrm{TPhB}^{-}$. 
ranged from $1.3 \times 10^{3} \mathrm{sec}^{-1}$ with no $3 \mathrm{PI}$ to $0.38 \times 10^{3} \mathrm{sec}^{-1}$ at $6.0 \times 10^{-5} \mathrm{M} 3 \mathrm{PI}$, and for TPhB ${ }^{-}$transport $k_{j}$ ranged between $15 \mathrm{sec}^{-1}$ in the absence of $3 P I$ and $24 \mathrm{sec}^{-1}$ when $8.0 \times 10^{-5} \mathrm{M} 3 \mathrm{PI}$ was present.

The transferred charge density as a function of applied voltage was fit to Eq. (5) for several membranes formed in the absence of 3PI, and it was found that the correlation was best when $b=0.72$ for $\mathrm{DPA}^{-}$ and when $b=0.83$ for $\mathrm{TPhB}^{-}$so these values of $b$ were used in all curve fits. For TPhB ${ }^{-}$the correlation was very good (typically correlation coefficient $>.96$ ) even up to $250 \mathrm{mV}^{\text {but }}$ for $\mathrm{DPA}^{-}$the correlation was greatly decreased if all voltages were included in the fit. This occurred in spite of the fact that the steady state current for DPAwas measured at each voltage and subtracted from the total current so that $I_{0}$ was only due to transient current relaxation. The low correlation at high voltages is probably due to the existence of some charge transfer mechanism other than lipophilic anion transport across the membrane interior (Ketterer et al., 1971). Bruner (1975) investigated $\mathrm{DPA}^{-}$transport across exceptionally stable synthetic lipid bilayers using large applied voltages, $200-400 \mathrm{mV}$, and found the current decay at higher voltages to be distinctly nonexponential. In determining $Q_{\text {ads }}$ for $D A^{-}$we have used $\triangle Q$ only for voltages less than or equal to $175 \mathrm{mV}$, in which region the fit to Eq. (5) was nearly as good as for $\mathrm{TPhB}^{-}$.

Using the values of $b$ listed above, $Q_{a d s}$ for $\mathrm{TPhB}^{-}$and $\mathrm{DPA}^{-}$was determined from Eq. (5) at each 3PI concentration. The results are displayed in Fig. 18; error bars are the standard deviation of $4-7$ membranes and the equivalent lipophilic anion density $\mathrm{N}_{\text {ads }}$ in moles/m $\mathrm{m}^{2}$ 


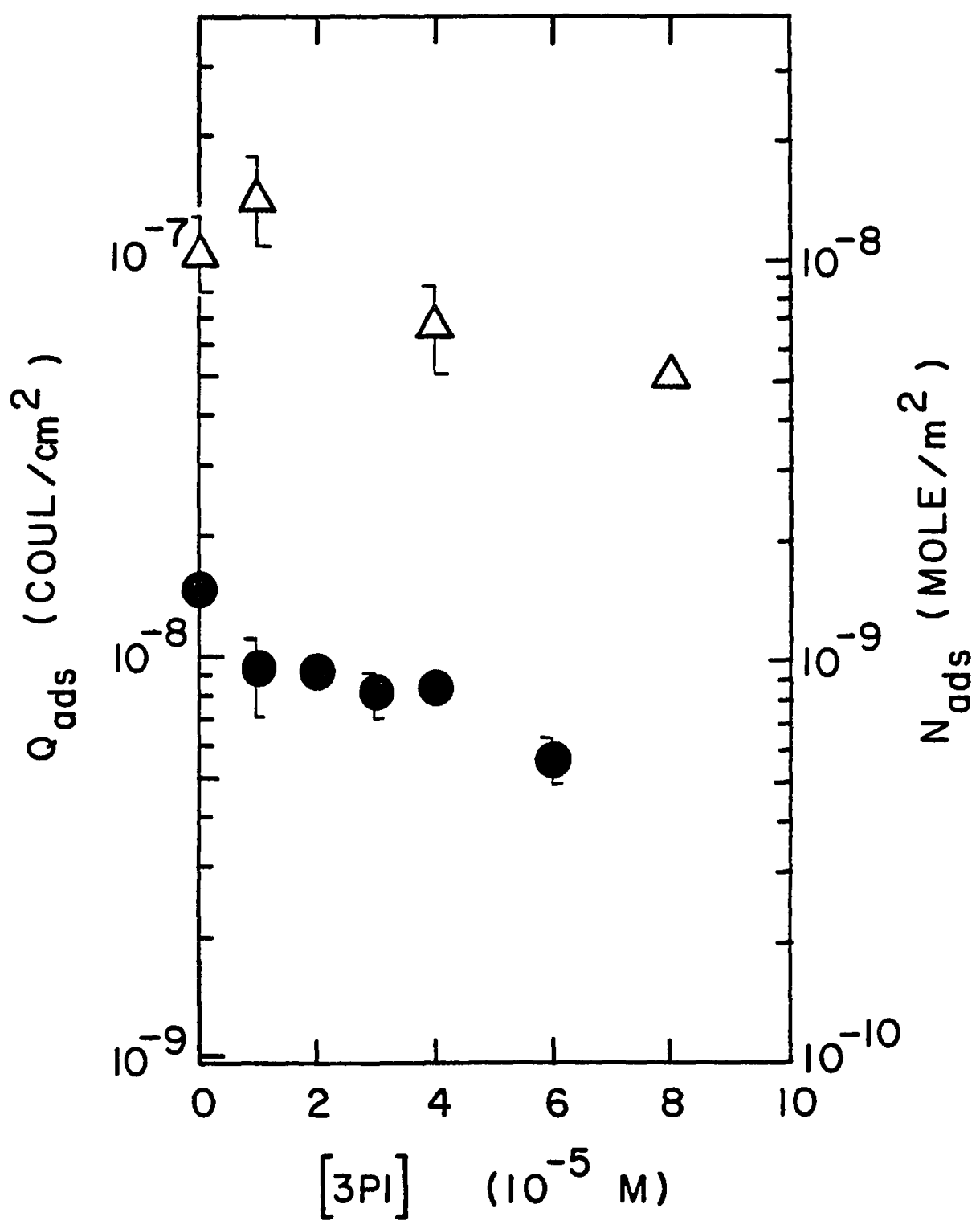

Figure 18. Adsorbed surface charge density (left-hand ordinate) and adsorbed surface number density (right-hand ordinate) as a function of 3-phenylindole concentration: $\mathrm{DPA}^{-}, \triangle \mathrm{TPhB}^{-}$. 
is given on the right-hand ordinate. It can be seen from this figure that the adsorbed surface charge density, which is composed only of the lipophilic anion density, did not change very much as the aqueous 3PI concentration increased. For DPA , $Q_{\text {ads }}$ was $14.5 \times 10^{-9} \mathrm{coul} / \mathrm{cm}^{2}$ $\left(N_{\text {ads }}=1.5 \times 10^{-9}\right.$ moles $\left./ \mathrm{m}^{2}\right)$ in the absence of $3 P I$ and decreased with increasing $[3 P I]$, having a value of $5.5 \times 10^{-9} \mathrm{cou} 1 / \mathrm{cm}^{2}\left(\mathrm{~N}_{\mathrm{ads}}=\right.$ $5.7 \times 10^{-10}$ moles $/ \mathrm{m}^{2}$ ) at $6.0 \times 10^{-5} \mathrm{M} 3 \mathrm{PI}$. For TPhB ${ }^{-}, Q_{\text {ads }}$ was $10.5 \times 10^{-8} \mathrm{coul} / \mathrm{cm}^{2}\left(\mathrm{~N}_{\mathrm{ads}}=1.1 \times 10^{-8} \mathrm{~mole} / \mathrm{m}^{2}\right)$ in the absence of 3PI and decreased slightly with increasing [3PI], having a value of $Q_{\text {ads }}=5.0 \times 10^{-8}$ cou $1 / \mathrm{cm}^{2}\left(N_{\text {ads }}=5.2 \times 10^{-9} \mathrm{~mole} / \mathrm{m}^{2}\right)$ at the highest concentration of $8.0 \times 10^{-5} \mathrm{M} 3 \mathrm{PI}$. The partition coefficients $\beta\left(B=N_{a d s} / C\right)$ derived from these measurements are listed in Table II.

TABLE II

PARTITION COEFFICIENT FOR LIPOPHILIC ANIONS IN THE PRESENCE OF 3-PHENYLINDOLE

\begin{tabular}{lcc}
\hline Anion & $\begin{array}{c}{[3 \mathrm{PI}]} \\
\left(10^{-5} \mathrm{M}\right)\end{array}$ & $\begin{array}{c}\beta \\
\left(10^{-4} \mathrm{~m}\right)\end{array}$ \\
\hline Dipicrylamine & 0 & $1.53 \pm .11$ \\
& 1.0 & $0.97 \pm .21$ \\
& 2.0 & $0.95 \pm .07$ \\
& 3.0 & $0.84 \pm .09$ \\
& 4.0 & $0.85 \pm .01$ \\
Tetraphenylborate & 6.0 & $0.57 \pm .06$ \\
& 0 & $1.07 \pm .22$ \\
& 1.0 & $1.46 \pm .37$ \\
& 4.0 & $0.70 \pm .17$ \\
& 8.0 & $0.52 \pm .05$ \\
\hline
\end{tabular}


In summary, our results were: 1) a decrease in $G_{0}(0)$ for both $\mathrm{DPA}^{-}$and $\mathrm{TPhB}^{-}$with increasing $\left.[3 \mathrm{PI}], 2\right)$ an increase in $\tau(0)$ for $\mathrm{DPA}^{-}$ and a slight decrease in $\tau(0)$ for $\mathrm{TPhB}^{-}$with increasing [3PI], and 3) a decrease in $Q_{\text {ads }}$ (and $\beta$ ) for both $\mathrm{DPA}^{-}$and $\mathrm{TPhB}^{-}$with increasing [3PI].

\section{DISCUSSION}

The results for the effect of 3PI on 1ipophilic anion transport were unexpected, showing only small magnitude effects and containing a contradiction in the direction of the change in $\tau(0)$. Based on the increase of $\mathrm{TPhA}^{+}$conductivity (Chapter III), the decrease in monolayer surface potential (Chapter II), and the increase in nonactin$\mathrm{K}^{+}$conductivity (Chapter $\mathrm{V}$ ), we had reason to believe that $3 \mathrm{PI}$ created an additional dipole potential at the membrane/aqueous interface which decreased the potential energy barrier for cation transport indicated by $\Delta F_{g}$ in $F i g . ~ 14$. This corresponds to an increase in $\Delta F_{g}$ for anion transport and hence would imply by Eq. (13) that lipophilic anion conductivity should be greatly reduced in the presence of 3PI; however, we found only a very small decrease in $G_{0}(0)$ for both PPhB $^{-}$and $D P A^{-}$.

Recall that $\Delta F_{g}$ is a combination of $\Delta F_{j}$ and $\Delta F_{p}$ (refer to Fig. 14). With the transient current technique used in the lipophilic anion studies, it is possible to detect changes in $\Delta F_{p}$ by noting changes in $Q_{a d s}$, and to detect changes in $\Delta F_{i}$ by noting changes in $\tau(0)$, but only if electrostatic changes in barrier height are the main effect of 3PI. Depending on the precise location of the dipole 
potential change induced by $3 P I$, it is conceivable that $\Delta F_{j}$ could be increased and $\Delta F_{p}$ could be decreased in the presence of $3 P I$, or that $\Delta F_{i}$ could be increased while $\Delta F_{p}$ remained unaffected. It is difficult to imagine a location for the potential change such that $\Delta F_{p}$ is decreased while $\Delta F_{j}$ is decreased, since the electric field is essentially constant across the bilayer interior. If we assume for the moment that electrostatic changes in $\Delta F^{\prime} s$ are the only effect of $3 P I$, then according to our experimental results, the increase in $\tau(0)$ for $D P A^{-}$ implies an increase in $\Delta F_{i}$ yet the decrease in $\tau(0)$ for TPhB- implies a decrease in $\Delta F_{j}$ due to the presence of $3 P I$. This contradiction, in conjunction with the unexpectedly small decreases in $G_{0}(0)$ led us to conclude that nonelectrostatic effects are contributing to the change in lipophilic ion transport. Therefore, we have quantified the effect of 3PI using the formalism developed by Szabo (1976) to analyze the effect of cholesterol on ion transport. This formalism was outlined in a previous section and the main results given in Eqs. (15) and (18).

The separation of electrostatic and non-electrostatic effects due to 3PI was done using Eqs. (15) and (18) and the results shown in Table III. In all cases $\bar{G}^{+}$was calculated from $\mathrm{TPhA}^{+}$steady state conductivity (Chapter III); $\mathrm{G}^{-}$was calculated separately for DPA and $\mathrm{TPhB}^{-}$. Since $\mathrm{TPhA}^{+}$conductivity in the absence of 3PI was small $\left(\sim 5 \times 10^{-8} \mathrm{~s} / \mathrm{cm}^{2}\right)$ there was a large error $\left( \pm 1.5 \times 10^{-8} \mathrm{~s} / \mathrm{cm}^{2}\right)$ in the reference conductivity $[\mathrm{G} *(0)$ in $\mathrm{Eq} .(14)]$ used to calculate relative conductivity $\overline{G^{+}}$. For this reason the ratios and products of conductivities should be taken only as approximations. Nevertheless, 
TABLE III

COMPARISON OF ELECTROSTATIC AND NON-ELECTROSTATIC FACTORS

IN MODIFICATION OF LIPOPHILIC ION

TRANSPORT BY 3-PHENYLINDOLE

\begin{tabular}{ccccc}
\hline Lipophilic anion & $\begin{array}{c}{[3 \mathrm{PI}]} \\
(\mu \mathrm{M})\end{array}$ & $\exp \left(-\mathrm{F} \Delta V_{\mathrm{d}} / \mathrm{RT}\right)$ & $\begin{array}{c}\Delta V_{\mathrm{d}} \\
(-\mathrm{mV})\end{array}$ & $f(\bar{u})$ \\
\hline DPA $^{-}$ & 10 & 5.1 & 41 & 1.8 \\
& 20 & 9.3 & 56 & 2.1 \\
& 30 & 11.6 & 62 & 2.1 \\
& 40 & 21.6 & 77 & 3.6 \\
TPhB $^{-}$ & 10 & 3.1 & 28 & 3.0 \\
& 40 & 10.6 & 60 & 7.4 \\
\hline
\end{tabular}

The factor $\exp (-F \Delta V, R T)$ is calculated by $E q .(15)$ and the factor $f(\bar{u})$ is calculated by Eq. (18).

it is possible to deduce several trends in the data. The additional dipole potential $\Delta V_{d}$ due to the presence of $3 P I$ as determined by this method was $-35 \pm 6 \mathrm{mV}$ at $10 \mu \mathrm{M} 3 \mathrm{PI}$ and $-68 \pm 8 \mathrm{mV}$ at $40 \mu \mathrm{M} 3 \mathrm{PI}$. The electrostatic factor $\exp \left(-F \Delta V_{d} / R T\right)$ was about the same magnitude as the non-electrostatic factor $f(\bar{u})$ for $\mathrm{TPhB}^{-}$transport, both factors increasing from 3 at $10 \mu \mathrm{M} 3 \mathrm{PI}$ to about 9 at $40 \mu \mathrm{M}$ 3PI. In the case of $\mathrm{DPA}^{-}$, both the electrostatic factor and the non-electrostatic factor also increased with increasing [3PI] but the electrostatic factor increased to a greater extent. Thus for $\mathrm{DPA}^{-}$, at $10 \mu \mathrm{M} 3 \mathrm{PI}$, the electrostatic factor was 5.1 and the non-electrostatic factor was 1.8 while at $40 \mu \mathrm{M} 3 \mathrm{PI}$, the electrostatic factor was 22 and the nonelectrostatic factor only 3.6. These results using positively and negatively charged lipophilic ions confirm our suspicion that 
non-electrostatic effects played a large role in the modification of lipophilic ion transport by $3 P I$.

Our result that both $G_{0}(0)$ and $Q_{a d s}$ show decreases of less than an order of magnitude in the presence of $3 P I$ indicates that the decrease in conductivity can be attributed almost entirely to a decrease in the adsorbed density. The small decrease in $Q_{a d s}$ in the presence of $3 \mathrm{PI}$ is consistent with the adsorption plane for 3PI being mostly interior to (on the bilayer side of) the adsorption plane for the lipophilic anions. The translocation step of the transport process, which is related to $k_{j}$ and $\tau$, is apparently being influenced by both electrostatic and non-electrostatic factors which nearly cancel each other out. On the other hand, these two factors would complement each other for lipophilic cation transport, both of them causing an increased conductivity.

The electrostatic factor causing a decrease in 1 ipophitic anion conductivity is readily explained as an additional dipole potential $\Delta V_{d}$ near the interface. The comments on the interpretation of monolayer $\delta(\Delta V)$ given in the Discussion section of Chapter II are applicable also to $\Delta V_{d}$ given in Table III. In particular, $\Delta V_{d}$ can be interpreted as due to $3 \mathrm{PI}$ dipoles or due to a change in the density or orientation of 1 ipid dipoles. It is noteworthy that $\Delta V_{d}$ is of smaller magnitude than $\delta(\Delta V)$ (see Fig. 6) at all 3PI concentrations, a phenomenon which was discussed in Chapter III in terms of a discrete charge or discrete dipole model. $\Delta V_{d}$ is a micropotential sensed by the lipophilic ion whereas $\delta(\Delta V)$ is a macropotential sensed by an electrode far from the monolayer so the theory predicts that $\Delta V_{d}$ 
should be smaller than the change in monolayer surface potential.

The non-electrostatic factor causing an increase in 1 ipophilic anion conductivity and in lipophilic cation conductivity is probably due to either a higher ion mobility in the membrane interior caused by a lower membrane viscosity when $3 P I$ is present or to a decrease in thickness of the bilayer caused by the presence of 3PI. Further investigation could reveal whether either of these effects is actually occurring. For example, the thickness of the bilayer can be measured using established techniques based on bilayer capacitance (Hanai, Haydon and Taylor, 1965; Benz, Fröhlich and Läuger, 1977). The viscosity of bilayer lipid membranes can be inferred from the viscosity of other lipid membrane models. For instance, the viscosity of liposomes can be monitored by fluorescence spectroscopy (Shinitsky et al., 1971) or nuclear spin resonance (Ahmad and Mellors, 1978); a slight modification of the set-up described in Chapter II makes possible the measurement of lipid monolayer viscosity (Gaines, 1966).

There is one other possibility which must be considered in attempting to explain the small magnitude of the effect of 3PI on 1ipophilic anion transport. It is possible that although 3PI adsorbs strongly to unmodified PC/cholesterol monolayers (Chapter II) and apparently adsorbs strongly to 3PI bilayers in the presence of $\mathrm{TPhA}^{+}$ (Chapter III), 3PI is inhibited from adsorbing to PC/cholesterol bilayers in the presence of $\mathrm{TPhB}^{-}$or $\mathrm{DPA}^{-}$. In order to test this hypothesis it would be necessary to develop a direct way of measuring the amount of 3PI adsorbed to the bilayer and then see how the presence of varying concentrations of lipophilic anion altered this adsorption. 


\section{CHAPTER V}

\section{ENHANCEMENT OF NONACTIN-K ${ }^{+}$STEADY STATE TRANSPORT BY 3-PHENYLINDOLE}

\section{MECHANISM OF NONACTIN TRANSPORT}

As discussed in Chapter I, the study of changes in electrical conductivity of lipid bilayer membranes induced by the macrotetralide antibiotics and valinomycin has increased our understanding of the carrier mechanism of ion transport. These antibiotics have a cagelike structure (nonactin structure is shown in Fig. 19) composed of a hydrophilic interior, where the ion resides, and a hydrophobic exterior. Transport of ions by the antibiotic carriers is ion selective, with $\mathrm{K}^{+}$and $\mathrm{NH}_{4}^{+}$being transported to a much greater extent than $\mathrm{Na}^{+}$; this ion selectivity has its basis in the requirement that the ion must fit inside the cage.

Experimental results for these compounds support a carrier transport scheme in which one ion is transported across a lipid membrane by one antibiotic molecule and then released to the aqueous solution, leaving the neutral antibiotic molecule free to diffuse back across the membrane (Ciani, Eisenman and Szabo, 1969; Hladky, 1972). The basic carrier mechanism can be modified to include situations in which the ion/carrier complex is formed in the aqueous solution or in the membrane (Hladky, 1972), in which unstirred layers play a role (Hladky, 1973), and in which the carrier is a charged molecule 


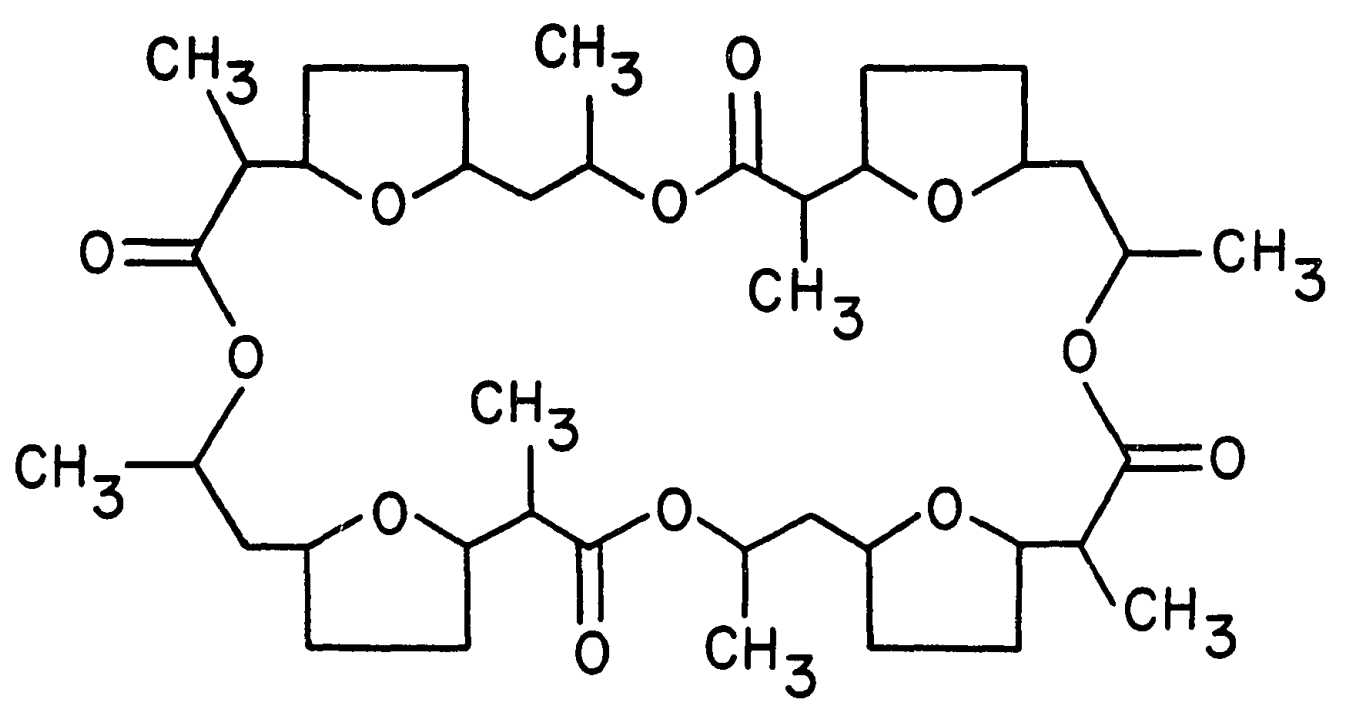

Figure 19. Chemical formula of nonactin. 
(Hladky, 1972). Hladky (1972) derives expressions for the flux with various assumptions that reflect possible locations and relative proportions of uncomplexed and complexed carrier in the bulk aqueous phase, the bilayer itself, and the Plateau border. In the experiment we put nonactin in both the aqueous phase $\left(10^{-7} \mathrm{M}\right)$ and in the membrane forming solution $\left(3 \times 10^{-5} \mathrm{M}\right)$ and only take measurements after a period of greater than half an hour, so that equilibrium of nonactin between aqueous phase, bilayer, and Plateau border was assured. Due to the low $\left[\mathrm{K}^{+}\right]$used in the experiment $(0.11 \mathrm{M})$, only few ion/carrier complexes are formed so the concentration of free carrier in the bilayer was a constant. The carrier transport scheme suitable with these experimental conditions is shown diagrammatically in Fig. 20. In that figure $N_{S}$ is the number density of free carrier, $N_{i s}$ is the number density of ion/carrier complexes and $a_{j}$ is the ion activity in the bulk aqueous solution. A single prime refers to the left side of the membrane while a double prime denotes the right side. Each step is characterized by a rate constant $k$ with appropriate subscript; a subscript $R$ refers to recombination of the complex and a subscript $D$ to dissociation of the complex while the free carrier is denoted by subscript $s$ and the ion/carrier complex by subscript is. Each step (complex translocation, complex formation, complex dissociation, and free carrier diffusion) occurs at a rate which reflects the energy barrier to be overcome at that stage of the transport. Notation is that of HTadky (1974).

The rate of change of the number densities at the left interface is given by 


\section{AQUEOUS BILAYER AQUEOUS}

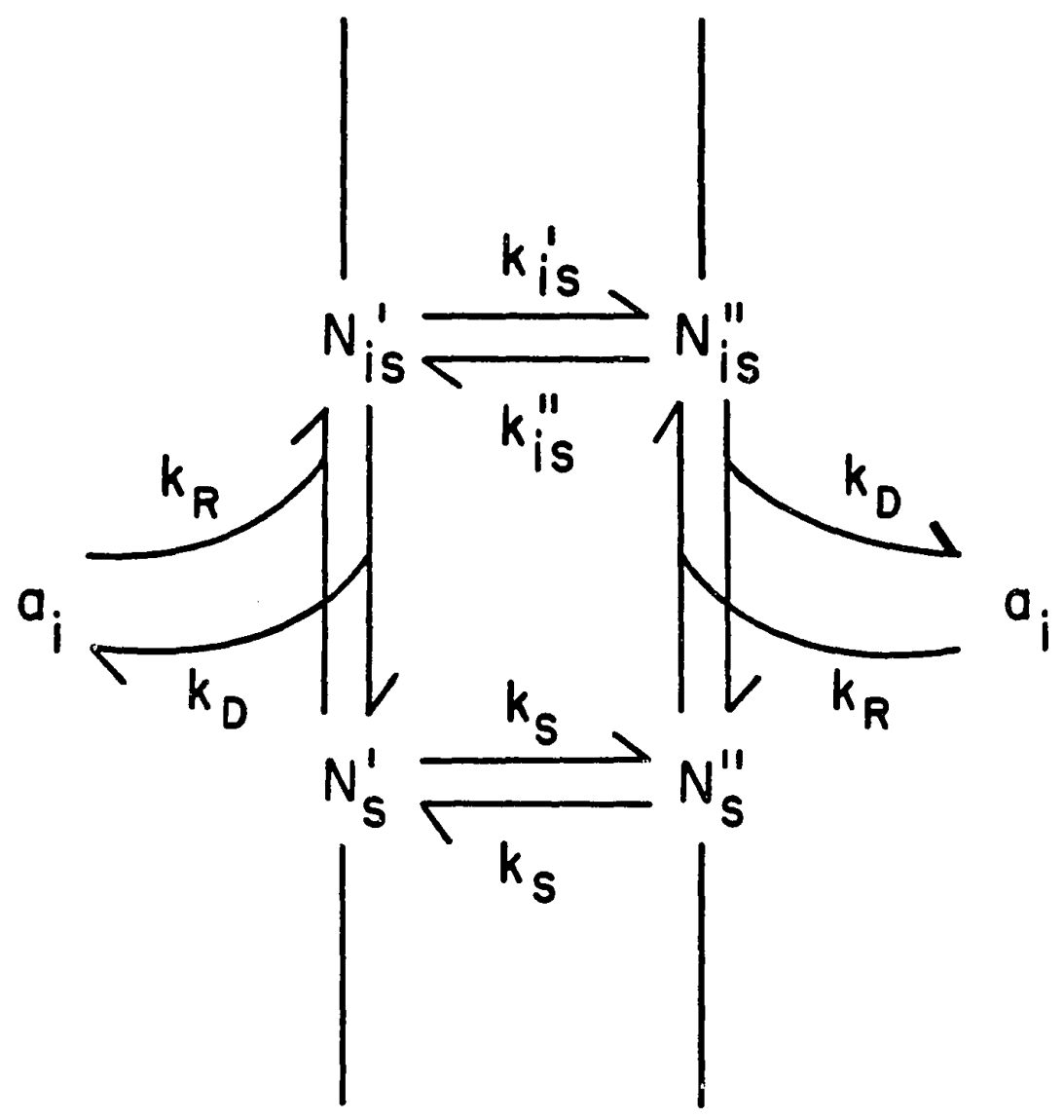

Figure 20. Kinetic model of nonactin- $K^{+}$transport. 


$$
\begin{gathered}
\frac{d N_{i s}^{\prime}}{d t}=-k_{i s}^{\prime} N_{i s}^{\prime}+k_{i s}^{\prime} N_{i s}^{\prime}-k_{D} N_{i s}^{\prime}+k_{R} a_{i} N_{S}^{\prime}, \\
\frac{d N_{s}^{\prime}}{d t}=-k_{s} N_{s}^{\prime}+k_{s} N_{s}^{\prime \prime}+k_{D} N_{i s}^{\prime}-k_{R} a_{i} N_{s}^{\prime},
\end{gathered}
$$

and at the right interface by

$$
\begin{aligned}
& \frac{d N_{i s}^{\prime \prime}}{d t}=+k_{i s}^{\prime} N_{i s}^{\prime}-k_{i s}^{\prime} N_{i s}^{\prime \prime}-k_{D} N_{i s}^{\prime \prime}+k_{R} a_{i} N_{s}^{\prime \prime}, \\
& \frac{d N_{S}^{\prime \prime}}{d t}=k_{s} N_{s}^{\prime}-k_{s} N_{s}^{\prime \prime}+k_{D} N_{i s}^{\prime \prime}-k_{R} a_{i} N_{s}^{\prime \prime}
\end{aligned}
$$

Although all of the rate constants may have some voltage dependence, $k_{R}$ and $k_{D}$ are assumed voltage independent since the voltage drop across the interfacial region where recombination and dissociation occur is much smaller than the voltage drop across the membrane interior. The rate constants for translocation are treated according to the usual Eyring assumption

$$
\begin{aligned}
& k_{i s}^{\prime}=k_{i s} \exp (u / 2), \\
& k_{i s}^{\prime \prime}=k_{i s} \exp (-u / 2),
\end{aligned}
$$

where $u$ is the reduced voltage defined in Chapter III and $k_{\text {is }}$ is the zero voltage value of both $k_{i s}^{\prime}$ and $k_{i s}^{\prime}$. After an initial ( $10 \mu \mathrm{sec}$ ) displacement current due to the charging of the lipid itself, the electrical current density, $J$, is $J=F\left(k_{i s}^{\prime} N_{i s}^{\prime}-k_{i s}^{\prime} N_{i s}^{\prime}{ }^{\prime}\right)$ when nonactin transports a monovalent cation. The solution of Eqs. (1) and (2) together with the voltage dependence for the translocation rate constants given in Eq. (3) leads to the following expression (Stark and Benz, 1971) for the steady state current density: 


$$
J=F d k_{i s} c_{i s}^{m} \frac{\sinh (u / 2)}{1+A \cosh (u / 2)} \text {, }
$$

where

$$
A=\frac{2 k_{i s}}{k_{D}}+\frac{k_{R} a_{j} k_{i s}}{k_{s} k_{D}},
$$

with additional notation that $F$ is the Faraday, $d$ is the thickness of the bilayer, and $c_{i s}^{m}$ is the concentration of ion/carrier complexes in the bilayer.

At zero applied voltage, equilibrium exists so that $N_{S}^{\prime}=N_{S}^{\prime \prime}=N_{S}$ and $N_{i s}^{\prime}=N_{i s}^{\prime \prime}=N_{i s}$. When the concentrations of complexed and free carrier are disturbed only slightly from their equilibrium values, the transport is said to be in the "equilibrium domain", a situation which corresponds to setting $A=0$ in Eq. (4). It is instructive at this point to compare $\mathrm{TPhA}^{+}$transport with nonactin- $\mathrm{K}^{+}$transport. For both types of transport, the current is initially determined only by the translocation step ( $k^{\prime}$ and $k^{\prime \prime}$ ' in Fig. 8; $k_{i s}^{\prime}$ and $k_{j s}^{\prime \prime}$ in Fig. 20). In the case of $\mathrm{TPhA}^{+}$, a steady current is maintained since $\mathrm{TPhA}^{+}$ions move rapidly across the interface and replenish the supply of $\mathrm{TPhA}^{+}$ in the membrane. In contrast, the replenishment of the ion/carrier complex for nonactin- $\mathrm{K}^{+}$transport requires a supply of both free neutral carrier and ion, which means that a steady current is dependent upon back diffusion of neutral carrier $\left(k_{S}\right)$ and upon dissociation $\left(k_{D}\right)$ and recombination $\left(k_{R}\right)$ of the complex. The interrelationships can lead to a situation in which the initial flux is not maintained, the number densities become different at the two interfaces, and equilibrium no longer exists. This non-equilibrium situation corresponds to nonzero $A$ in Eq. (4). 
The steady state conductivity results will be given in terms of conductivity $G(V)[G(V)=J / V$ where $V$ is the applied voltage] and of the ohmic limit of conductivity $G(0)\left[G(0)=(J / V)_{V \rightarrow 0}\right]$. From Eq. (4) the relationship (Stark and Benz, 1971) between $G(0)$ and the rate constants is

$$
G(0)=\frac{F^{2} d k_{i s}}{2 R T} \frac{c_{i s}^{m}}{1+A} .
$$

Additional information can be obtained from the manner in which conductivity depends on the applied voltage. As outlined in Chapter III, it can be shown that the normalized conductivity is

$$
\frac{G(V)}{G(0)}=\frac{2}{u}(1+A) \exp \left(-\omega u^{2}\right) \frac{\sinh (u / 2)}{1+A \exp \left(-\omega u^{2}\right) \cosh (u / 2)},
$$

where the kinetic limitation parameter $A$ for nonactin- $K^{+}$transport is defined in Eq. (5) of this chapter. When translocation of the ion/ carrier complex across the membrane is the rate determining step, parameter $A$ is zero. In that circumstance nonactin- $\mathrm{K}^{+}$transport is analogous to the transport of $\mathrm{TPhA}^{+}$. Parameter $A$ for nonactin- $\mathrm{K}^{+}$transport is a more complex expression than parameter $A$ for lipophilic ion transport, which is a consequence of the additional steps for the carrier mechanism; note, however, that both expressions contain ratios of rate constants. For nonactin $-\mathrm{K}^{+}$transport, larger values of parameter A correspond to situations in which one of the steps other than translocation is the rate determining step, and in these situations the transport is referred to as being kinetically limited. From the definition of parameter $A$ given in Eq. (5) it can be seen that at low ion activity (small $a_{j}$ ) the transport can be kinetically limited by slow dissociation (large $k_{i s} / k_{D}$ ). At higher ion activities the 
carrier can be nearly all in the complexed form so that the transport can become kinetically limited by slow back diffusion of the free carrier (large $k_{R} a_{j} k_{i s} / k_{D} k_{s}$ ) [Hladky, 1979]. In Fig. 21, Eq. (7) is plotted for several values of A to show the effect of increasing kinetic limitations, i.e., larger values of parameter $A$. The value of $\omega$ depends on the thickness of the membrane; in the calculations for Fig. 21 a value of $\omega=.007$ was used to reflect the thickness of the membranes used in our experiment.

\section{MATERIALS AND METHODS}

The materials and experimental procedures used for steady state nonactin conductivity measurements were the same as those described in Chapter III with modifications given below; the circuit is shown in Fig. 10. The aqueous solution consisted of $0.1 \mathrm{M} \mathrm{KCl}^{\text {, buffer }} \mathrm{B}^{-3}$, $10^{-7} M$ nonactin (gift of Dr. B. Stearns, Squibb Institute) from ethanolic solution, plus the desired amount of 3PI from a stock solution in acetone. The concentration of acetone in the final solution never exceeded $0.5 \%$; within the 1 imits of experimental error, this concentration of acetone did not change the conductivity of control membranes without 3PI. In a separate set of experiments the 3PI was added to the membrane forming solution instead of to the aqueous solution.

For the $\mathrm{pH}$ dependence studies it was necessary to use sodium hydroxide and a stronger buffer. Therefore the aqueous solution for these studies consisted of $0.04 \mathrm{M} \mathrm{KCl}, 10^{-7} \mathrm{M}$ nonactin, $25 \mu \mathrm{M} \mathrm{3PI}$, and a buffer $\left(M B^{-1}\right)$ of potassium phosphate:potassium citrate:boric 


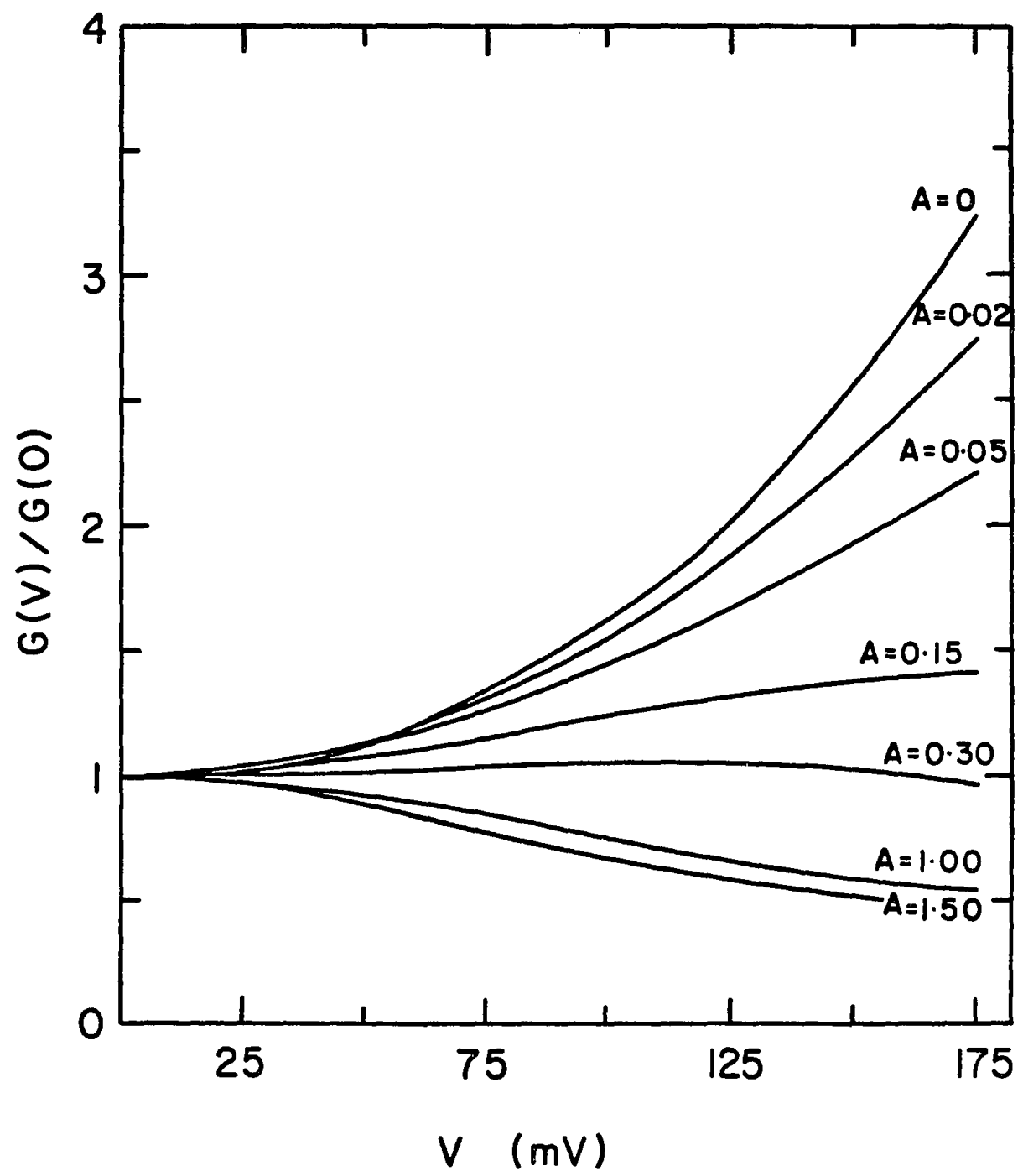

Figure 21. Theoretical curves of normalized conductivity as a function of voltage [Eq. (7) with $\omega=0.007]$ for various values of parameter A. 
acid:sodium hydroxide $0.2 \mathrm{M}: 0.2 \mathrm{M}: 0.05 \mathrm{M}: 0.34 \mathrm{M}$. The $\mathrm{pH}$ was adjusted with $\mathrm{HCl}$ in all cases.

The magnitude of the conductivity is mainly dependent on the concentration of nonactin in the aqueous solution but in order to aid equilibration of nonactin between the membrane and the aqueous solution, the membrane forming solution also contained $3 \times 10^{-5} \mathrm{M}$ nonactin. In the absence of 3PI the membranes took 20 - 50 minutes to thin but even small concentrations of $3 P I$ reduced the thinning time to less than 10 minutes. Measurements were taken after the current reached a steady value, typically 30 - 45 minutes after the membrane became black.

\section{RESULTS AND DISCUSSION}

In Fig. 22 the zero voltage conductivity due to nonactin- $K^{+}$ transport is plotted as a function of aqueous concentration of 3PI; error bars are the standard deviation of at least eight membranes. G(0) increased with increasing [3PI] and was nearly three orders of magnitude larger in the presence of $8.0 \times 10^{-5} \mathrm{M} 3 P I\left[G(0)=2.2 \times 10^{-3}\right.$ $\left.\mathrm{S} / \mathrm{cm}^{2}\right]$ than in the absence of $3 P I\left[G(0)=2.9 \times 10^{-6} \mathrm{~s} / \mathrm{cm}^{2}\right]$. To aid in comparing the effect of 3PI on ion/carrier transport with the effect of 3PI on lipophilic ion transport, these data are shown in Fig. 23 as relative conductivities $\bar{G}[\bar{G}=G(0) / G *(0)$ where $G *(0)$ is the zero voltage conductivity in the absence of $3 P I]$, along with $\bar{G}^{\mp}$ for $\mathrm{TPhA}^{+}$(derived from the data shown in Fig. 11) and $\bar{G}^{-}$for the two lipophilic anions (derived from data shown in Fig. 16). The largest uncertainties are in $\overline{\mathrm{G}}$ for $\mathrm{TPhA}^{+}$since conductivities were smallest for that compound. It is obvious that 3PI enhances conductivity of 


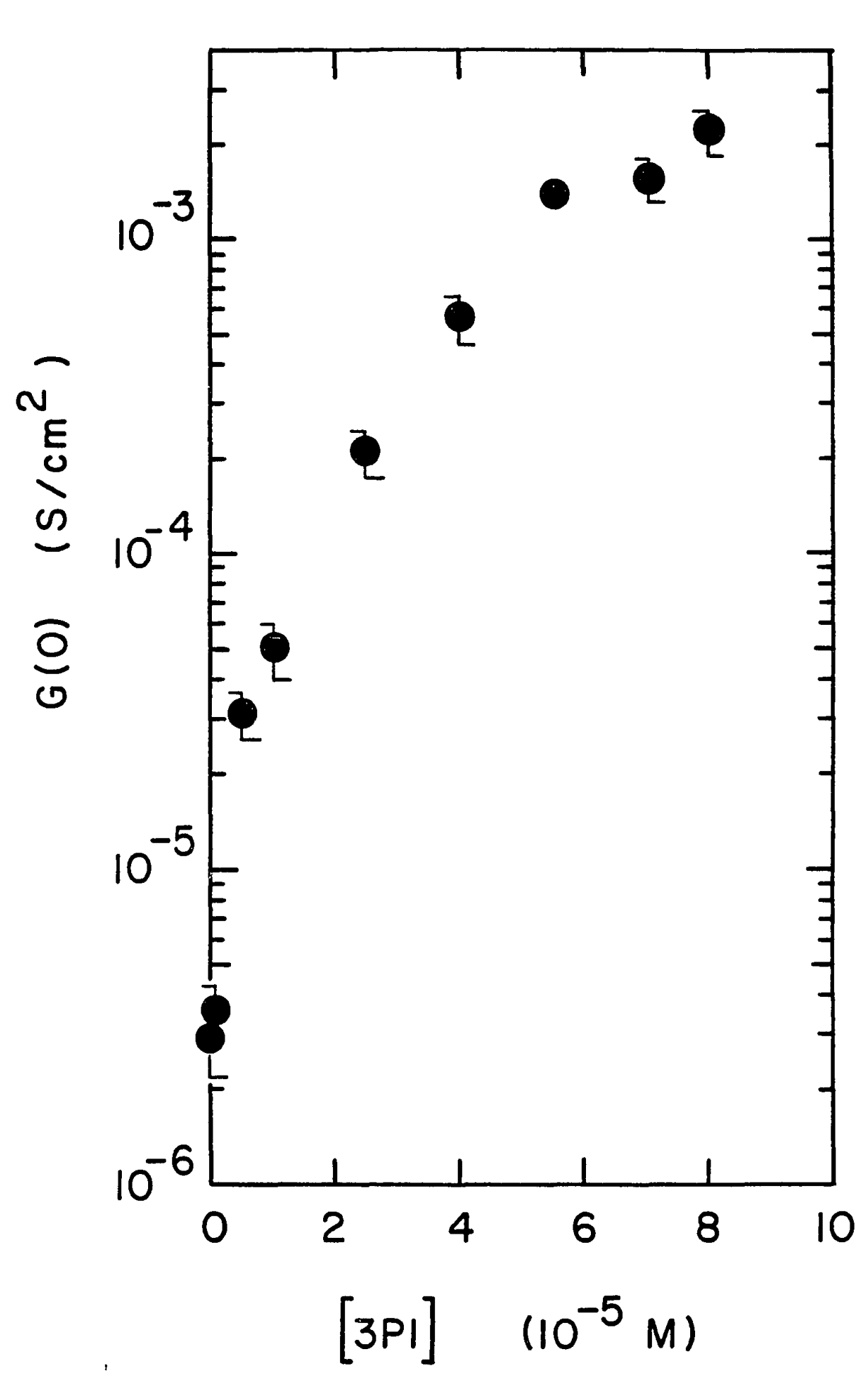

Figure 22. Nonactin $-K^{+}$zero voltage conductivity as a function of 3-phenylindole concentration in the aqueous solution. Aqueous solution also contained $10^{-7} \mathrm{M}$ nonactin, buffer $\mathrm{B}^{-3},\left[\mathrm{~K}^{+}\right] 0.11 \mathrm{M}$. 


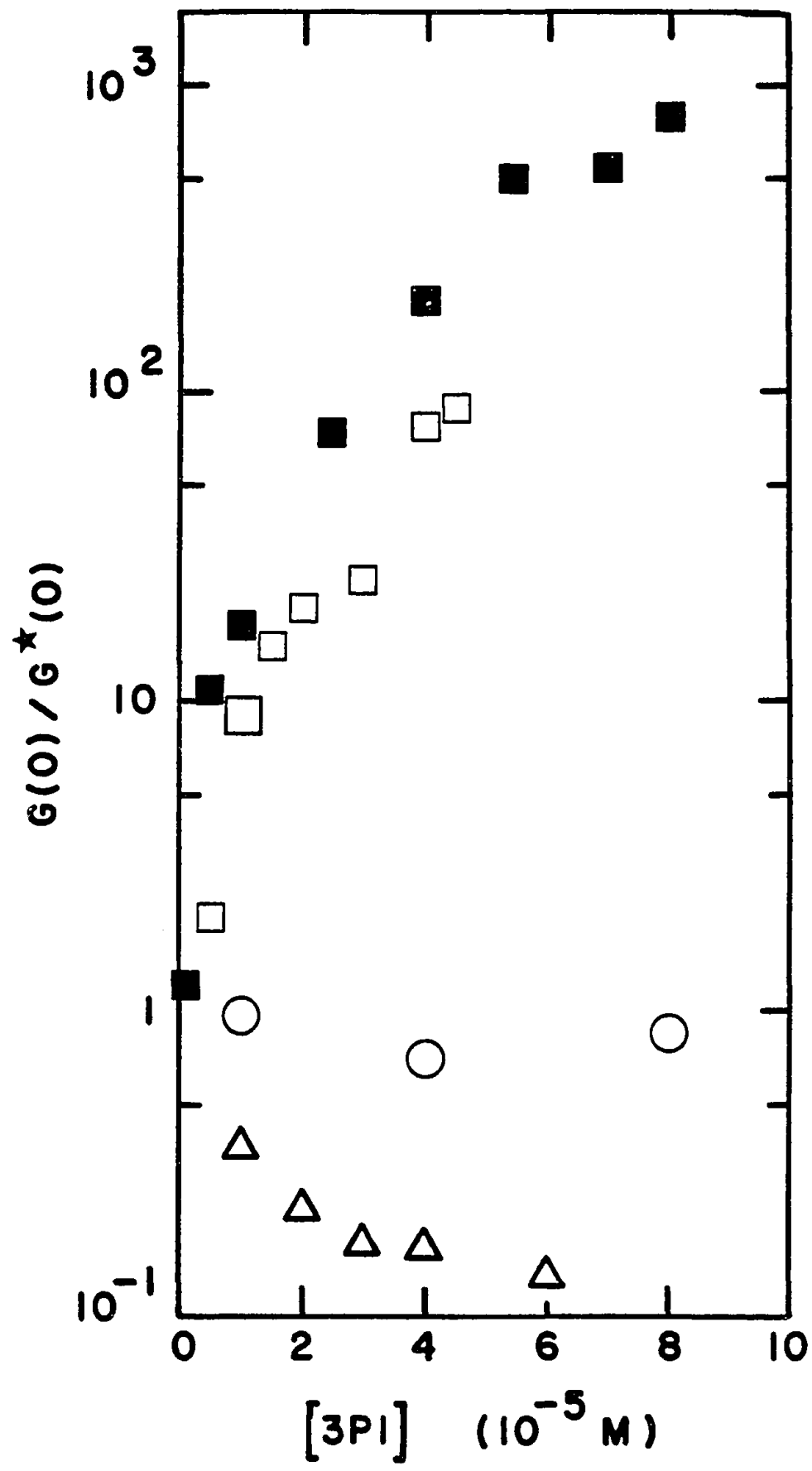

Figure 23. Relative conductivity as a function of 3-phenylindole concentration: nonactin- $\mathrm{K}^{+}, \square \mathrm{TPhA}^{+}, \bigcirc \mathrm{TPhB}^{-}, \triangle \mathrm{DPA}^{-}$. 
cations irrespective of whether the transport is by carrier mechanism (nonactin) or directly by lipophilic ion $\left(\mathrm{TPhA}^{+}\right)$, and that 3PI inhibits transport of anionic species ( $\left.\mathrm{TPhB}^{-}, \mathrm{DPA}^{-}\right)$. This is consistent with the observation discussed earlier that $3 P I$ changes the dipole potential of the aqueous/membrane interface, making the membrane interior more negative. The enhancement of the carrier conductivity appears to be greater than enhancement of the lipophilic cation conductivity, but the slight difference could easily be due to the size and shape of the molecules involved rather than the extra steps in the carrier transport process. As discussed in Chapter IV, we found that 3PI influenced the transport of lipophilic ions by non-electrostatic effects such as decreasing the viscosity of the bilayer. Since nonactin is more bulky than the lipophilic ion, it is likely that decreased viscosity would enhance the conductivity of nonactin- $k^{+}$even more than it enhances $\mathrm{TPhA}^{+}$conductivity. Thus we conclude that the enhancement of nonactin- $\mathrm{K}^{+}$conductivity by $3 P I$ is due to a combination of electrostatic and non-electrostatic effects.

Nonactin- $\mathrm{K}^{+}$transport itself is $\mathrm{pH}$ independent from $\mathrm{pH} 2$ - 11 (Smejtek and Pautis-Illangasekare, 1979a). The enhancement of conductivity by $3 \mathrm{PI}$ was also found to be $\mathrm{PH}$ independent over a wide range as shown by the plot of $G(0)$ versus $\mathrm{pH}$ in Fig. 24. This result is not unexpected since $3 P I$ is in neutral form over the same $\mathrm{pH}$ range. The normalized conductivity at pH 6.9 is shown in Fig. 25 as a function of applied voltage. In comparing this figure with Fig. 21, we see that increasing [3PI] causes increasing kinetic limitations. The membranes had cholesterol mole fraction of 0.22 which implies a 


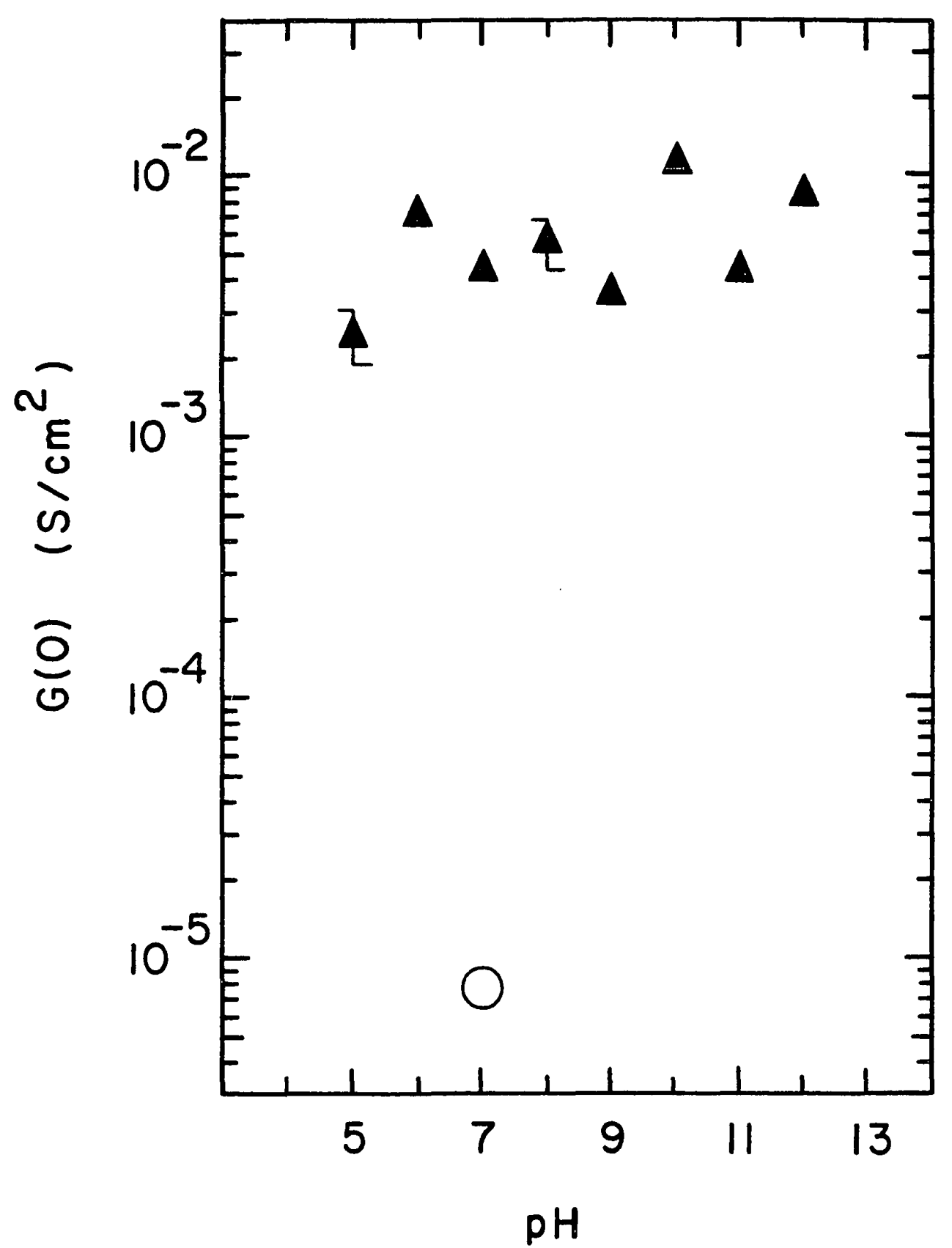

Figure 24. Nonactin- $\mathrm{K}^{+}$zero voltage conductivity as a function of $\mathrm{pH}$. Aqueous solution $10^{-7} \mathrm{M}$ nonactin, buffer $\mathrm{MB}^{-1},\left[\mathrm{~K}^{+}\right] 0.5 \mathrm{M} \bigcirc$ without 3-phenylindole, $\triangle$ with 25 M 3-phenylindole. 


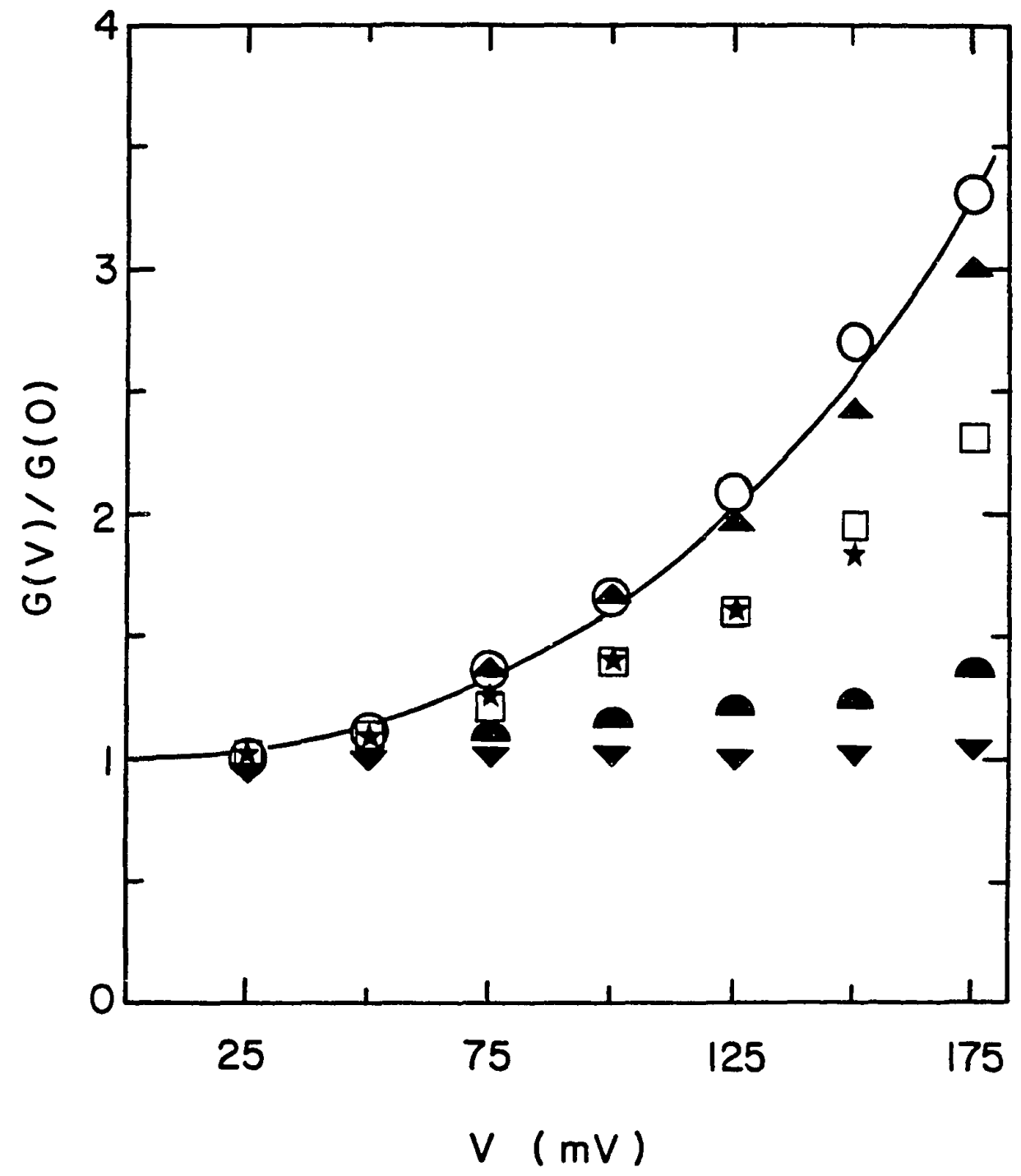

Figure 25. Nonactin- $\mathrm{K}^{+}$normalized conductivity as a function of applied voltage with the indicated concentration of 3-phenylindole in the aqueous solution: $00 \mu \mathrm{M}, \triangle 10 \mu \mathrm{M}, \square 25 \mu \mathrm{M}, \downarrow 40 \mu \mathrm{M}$, a $55 \mu \mathrm{M}, \nabla 80 \mu \mathrm{M}$. Solid line is Eq. (4) with $A=0$. 
thickness of $45 \AA$ (Hanai, Haydon and Taylor, 1965). Using $\omega=.007$ [based on Table I in Andersen and Fuchs (1975) relating thickness and w], the data in Fig. 25 were fit to Eq. (7) to obtain the values of parameter $A$ as listed in the upper half of Table IV. The lower half of the table is for a related experiment and will be discussed shortly. At small concentrations of $3 \mathrm{PI}$ in the aqueous solution, parameter $A$ was near 0 , indicating that ion/carrier complex translocation across the membrane was the slowest step in the process. At $[3 \mathrm{PI}] \geq 2.5 \times 10^{-5} \mathrm{M} 3 \mathrm{PI}$, parameter $A$ increased, and at the highest concentration of $8.0 \times 10^{-5} \mathrm{M}$ 3PI parameter A was .30, which corresponds (see Fig. 25) to a conductivity which is voltage-independent and represents a strong kinetic limitation. The greater enhancement of nonactin- $\mathrm{K}^{+}$transport over $\mathrm{TPhA}^{+}$ transport as shown in Fig. 23 may be related to the change in $k_{R}, k_{D}$ or $k_{s}$ implied by the changes in parameter $A$ for nonactin- $K^{+}$. In order to ascertain which step of the transport process is being affected, it would be of some help to determine parameter $A$ as a function of $\left[\mathrm{K}^{+}\right]$and thus obtain ratios of rate constants by the best fit to Eq. (5). It is interesting to note that kinetic limitations occur in the same $3 \mathrm{PI}$ concentration range in which the binding of $3 \mathrm{PI}$ to $\mathrm{PC} / \mathrm{cholesterol}$ monolayers shows saturation (Fig. 3 ).

From an environmental viewpoint a pesticide is more likely to be initially in the aqueous phases surrounding a cell or microorganism and in the experiments discussed so far, 3PI was placed in the aqueous solution. Since 3PI is hydrophobic some fraction of it is adsorbed to, or dissolved in, the lipid bilayer. In the next experiment 3PI was included in the membrane forming solution instead of in the aqueous phase and the conductivity measured in the usual way. The results 
are shown in Fig. 26 as $G(0)$ versus mole ratio of $3 P I$ to lipid (PC plus cholesterol) in the membrane forming solution. The mole ratio is probably an overestimate of the actual amount of 3PI in the bilayer since part of what was put into the membrane forming solution partitioned into the Plateau border and also into the large aqueous volume on either side of the bilayer. This escape was more pronounced at high $3 P I: 1$ ipid mole ratios as evidenced by the fact that conductivity decreased with time after the membrane became black when the mole ratio was 1.6. $G(0)$ increased with increasing $3 P I$ mole ratio and was more than two orders of magnitude greater in the presence of 3PI:lipid $=0.94\left[G(0)=4.1 \times 10^{-4} \mathrm{~s} / \mathrm{cm}^{2}\right]$ than in the absence of $3 P I$ $\left[G(0)=2.9 \times 10^{-6} \mathrm{~s} / \mathrm{cm}^{2}\right]$.

The conductivity is determined by the coricentration of $3 \mathrm{PI}$ in the bilayer, which is not known with certainty in this experiment or in the experiment in which $3 \mathrm{PI}$ is placed in the aqueous solution. However, it can be seen that an aqueous 3PI concentration of $(1-8) \times 10^{-5} \mathrm{M} 3 \mathrm{PI}$ produces the same change in conductivity (see Fig. 22) as that produced by mole ratios of $0.1-1.5$ of 3 PI:1ipid in the membrane forming solution, which is roughly equivalent to the amount in the bilayer itself. One indication that these mole ratios are an overestimate of the amount of $3 P I$ in the bilayer is that we found 3PI adsorbed to lipid monolayers of the same composition only up to a maximum of one 3PI molecule for every 2.8 lipid molecules (3PI: lipid $=0.36)$ as discussed in Chapter II. Nevertheless, these conductivity results reinforce the observation that 3PI strongly favors partition into the lipid phase. 


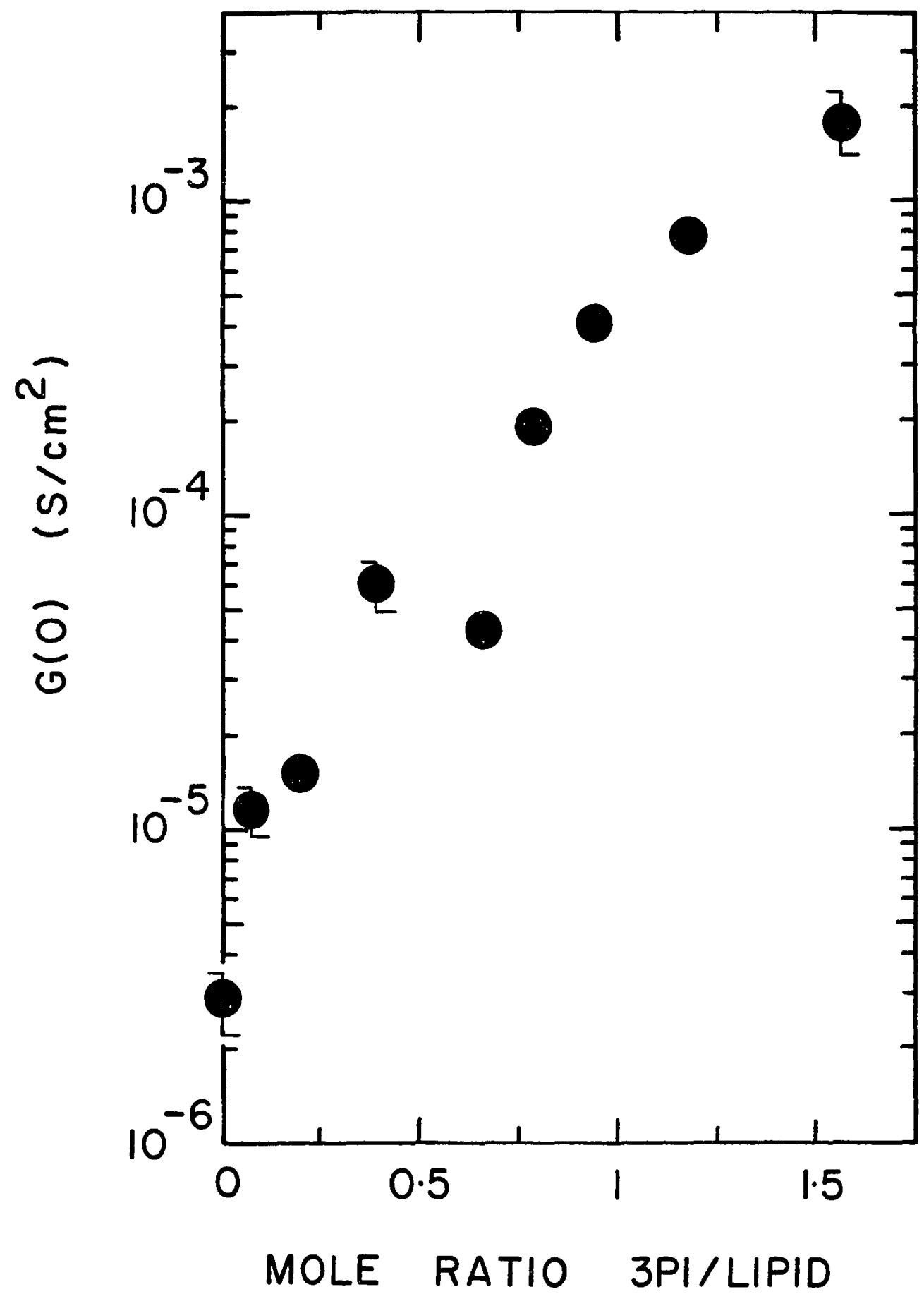

Figure 26. Nonactin- $\mathrm{K}^{+}$zero voltage conductivity as a function of 3-phenylindole concentration in the membrane forming solution. Aqueous solution $10^{-7} \mathrm{M}$ nonactin, buffer $\mathrm{B}^{-3},\left[\mathrm{~K}^{+}\right] 0.11 \mathrm{M}$. 
TABLE IV

KINETIC LIMITATION PARAMETER FOR NONACTIN-K ${ }^{+}$TRANSPORT IN THE PRESENCE OF 3-PHENYLINDOLE

\begin{tabular}{lll}
\hline & {$[3 P I]$} & Parameter A \\
& $\left(10^{-5} \mathrm{M}\right)$ & \\
3PI in aqueous solution & 0.0 & $-.01 \pm .02$ \\
& 0.05 & $-.02 \pm .03$ \\
& 0.5 & $-.01 \pm .02$ \\
& 1.0 & $.01 \pm .01$ \\
& 2.5 & $.05 \pm .02$ \\
& 4.0 & $.07 \pm .03$ \\
& 5.5 & $.18 \pm .03$ \\
& 7.0 & $.20 \pm .03$ \\
& 8.0 & $.30 \pm .03$ \\
\hline MPI in membrane & ratio $3 P I: 7$ ipid & Parameter A \\
forming solution & & \\
& 0.13 & $-.03 \pm .02$ \\
& 0.19 & $-.03 \pm .03$ \\
& 0.39 & $-.01 \pm .01$ \\
0.65 & $-.02 \pm .04$ \\
& 0.78 & $-.01 \pm .01$ \\
& 0.94 & $.05 \pm .02$ \\
& 1.2 & $.07 \pm .06$ \\
1.6 & $.14 \pm .04$ \\
\hline
\end{tabular}

Kinetic limitations were also observed when $3 P I$ was in the membrane forming solution as shown by values of parameter A given in the lower half of Table IV. Parameter A was near zero up to a mole ratio of $0.8-0.9$ and increased for mole ratios greater than that. Comparing this experiment with the one in which 3PI was placed in the aqueous solution (upper half of Table IV), note that for 3PI: lipid approximately $1: 1$ in the membrane forming solution parameter $A$ is between 0.05 and 0.07 . This same range of parameter $A$ occurred when 
[3PI] in the aqueous solution was between $25 \mu \mathrm{M}$ and $40 \mu \mathrm{M}$. The overall lower values of parameter $A$ when $3 P I$ was in the membrane forming solution are another indication that 3PI escapes from the bilayer. 


\section{CHAPTER VI}

\section{EFFECT OF 3-PHENYLINDOLE ON TRANSIENT NONACTIN-K ${ }^{+}$TRANSPORT}

\section{TRANSIENT COMPONENTS OF NONACTIN-K ${ }^{+}$TRANSPORT}

The kinetic scheme used to describe nonactin- $\mathrm{K}^{+}$transport was outlined in Chapter $V$ and used to obtain expressions for steady state conductivity. From steady state measurements one can obtain the combinations of rate constants $k_{j S} / k_{D}$ and $k_{R} a_{j} k_{i s} / k_{s} k_{D}$, but in order to determine all of the rate constants plus the partition coefficient of nonactin it is necessary to investigate the transient behavior of the current. The general time dependent solution (Stark et al., 1971) for carrier transport gives a steady state current plus a transient current with three components. The special case of a symetrical membrane results in the model described in Chapter $V$; the solution of Eqs. (1) and (2) in Chapter $V$ gives a steady state current plus two transient components whose time constants $\tau_{1}$ and $\tau_{2}$ are related to the rate constants (refer to Fig. 19) by the following:

$$
\begin{array}{lll}
\lambda_{1}=\tau_{1}^{-1}=a+b \quad & \text { (fast transient) } \\
\lambda_{2}=\tau_{2}^{-1}=a-b \quad, \quad & \text { (slow transient) }
\end{array}
$$

where

$$
\begin{aligned}
& a=\frac{1}{2}\left[2 k_{i s} \cosh (u / 2)+2 k_{s}+k_{R} a_{i}+k_{D}\right] \\
& b=\frac{1}{2}\left\{\left[2 k_{i s} \cosh (u / 2)-2 k_{s}-k_{R} a_{i}+k_{D}\right]^{2}+4 k_{D} k_{R^{2}}\right\}_{i}^{\frac{1}{2}} .
\end{aligned}
$$


The current relaxation amplitudes $\alpha_{1}$ and $\alpha_{2}$ are related to parameter $A$ defined in Eq. (5) of Chapter $V$ in the following manner:

$$
\begin{array}{ll}
\alpha_{1}=\frac{1}{2}[A \cosh (u / 2)]+B \quad, \quad \text { (fast transient) } \\
\alpha_{2}=\frac{1}{2}[A \cosh (u / 2)]-B, \quad \text { (slow transient) }
\end{array}
$$

where the parameter $B$ is also related to parameter $A$ by

$$
B=[\cosh (u / 2) \div 4 b]\left[A\left(2 k_{s}+k_{R} a_{i}+k_{D}-2 k_{i s} \cosh (u / 2)\right)-4 k_{i s}\right] \text {. }
$$

The current density $J(t)$ is thus given by the sum of the two components:

$$
J(t)=J_{\infty}\left[1+\alpha_{1} \exp \left(-t / \tau_{1}\right)+\alpha_{2} \exp \left(-t / \tau_{2}\right)\right],
$$

where $J_{\infty}$ is the steady state current density given by Eq. (4) of Chapter $V$. In the "equilibrium domain", i.e. with $A=0$, it can be seen from Eqs. (3) and (5) that $J(0)=J_{\infty}$; the initial and final currents are identical so there are no transients. From Eqs. (1) and (2) we can see that any conditions which greatly increase or decrease one of the rate constants while leaving the others unaffected will change the time constants for current decay. Furthermore, it is important to point out that by Eqs. (3) and (4), in circumstances in which parameter $A$ is large, an increased amplitude for at least one component of the current is predicted and a transient current should be observed in these circumstances.

Transient currents due to nonactin $-\mathrm{K}^{+}$, nonactin- $\mathrm{NH}_{4}^{+}$, trinactin- $\mathrm{K}^{+}$, and trinactin- $\mathrm{NH}_{4}^{+}$have been investigated by $\mathrm{Hladky}$ (1975) and those due to valinomycin- $\mathrm{Rb}^{+}$and val inomycin- $\mathrm{K}^{+}$by Benz, Fröhlich and Låuger (1977). In some cases for which a transient current was observed the current decay could be described well with a single time constant while for the valinomycin- $\mathrm{K}^{+}$transport the current 
seemed to be a combination of two relaxations. Hladky (1975) reported that no transient was observed for nonactin- $\mathrm{K}^{+}$if the $\mathrm{K}^{+}$concentration was $0.1 \mathrm{M}$ or less, but he was able to resolve a transient at $\mathrm{K}^{+}$concentrations of $1 M$ and $4 M$.

As discussed in Chapter V, 3PI caused a kinetic limitation of nonactin- $\mathrm{K}^{+}$transport. At a $\mathrm{K}^{+}$concentration of $0.1 \mathrm{M}$, we found that parameter $A$ increased from near zero in the absence of 3PI to approximately 0.3 in the presence of $8.0 \times 10^{-5} \mathrm{M} 3 \mathrm{PI}$. The theory outlined above predicts no transient current in the former condition and a transient current, with either one or two exponentially decaying components, in the latter condition.

\section{MATERIALS AND METHODS}

Bilayer lipid membranes were formed by the same technique and using the same materials listed in Chapter III. Initial current Io and time constant $\tau$ were measured by the procedure described in Chapter IV. The aqueous solution consisted of $0.1 \mathrm{M} \mathrm{KCl}$, buffer $B^{-3}$, $10^{-7} \mathrm{M}$ nonactin (gift of $B$. Stearns, Squibb Institute), plus the required amount of 3PI from a stock solution in acetone. To aid equilibration of nonactin between the membrane and the aqueous phases, $3 \times 10^{-5} \mathrm{M}$ nonactin was added to the membrane forming solution. Nonactin was stored as a concentrated ethanol solution.

The electrical noise level is affected by the capacitive load the $D / A$ converter must drive, so for these nonactin experiments a Teflon cup with a smaller aperture $(1.2 \mathrm{~mm}$ diameter) was used in order to have a smaller membrane capacitance. Some experiments were 
performed using sintered $\mathrm{Ag} / \mathrm{AgCl}$ electrodes (Type 140, Annex Instruments, Santa Ana, CA). For the experiments with no $3 P I$ and with $55 \mu M$ 3PI the steady state current at $25 \mathrm{mV}$ was monitored by an electrometer (Princeton Applied Research, Model 135, Princeton, NJ). Later modifications allowed steady state currents $I_{\infty}$ to be measured with the computerized set-up so that at a 3PI concentration of $80 \mu \mathrm{M}$ the steady state current at all applied voltages was obtained at the same time the transients were obtained. The relaxation amplitude $\alpha$ $\left[\alpha=\left(I_{0}-I_{\infty}\right) / I_{\infty}\right]$ could then be calculated.

\section{RESULTS AND DISCUSSION}

Table $V$ lists the relaxation time constants and relaxation amplitudes for a representative $\mathrm{PC} /$ cholesterol bilayer in the presence of nonactin and $80 \mu \mathrm{M} 3 \mathrm{PI}$. The relaxation was described well (correlation coefficient $>0.97$ ) by a single time constant. There was no other relaxation evident on other time scales up to 500 milliseconds. The best fit of the steady state data for this membrane to Eq. (7) in Chapter $V$ gave a value for parameter $A$ of 0.42 . Since the currents were fairly large (steady state current exceeded $10^{-7} \mathrm{Amp}$ ), it was possible to use small feedback resistance $(30 \mathrm{~K} \Omega$ ) and no feedback capacitance, resulting in the fastest amplifier response time possible. With these favorable operating conditions, it can be seen that the observed $\tau$ are only $\sim 4 \mu \mathrm{sec}$ and voltage-independent while the observed $\alpha$ are large. For comparison note that for nonactin- $\mathrm{K}^{+}$transport with $1 \mathrm{M} \mathrm{KCT}, \tau$ is $11 \mu \mathrm{sec}$ and $\alpha$ is $0.1-0.3$ (H1adky, 1975). Our observed relaxation is undoubtedly due to the charging of the bilayer itself 
rather than an indication of nonactin- $\mathrm{K}^{+}$transport. Thus, while the steady state measurements indicate that the transport of nonactin- $\mathrm{K}^{+}$ is kinetically limited (large A) by $80 \mu \mathrm{M} 3 \mathrm{PI}$ and definitely in the "nonequilibrium domain", the absence of a transient current must mean that the transition from equilibrium to steady state is below the resolution capabilities of the experimental set-up.

We have also performed experiments to determine whether a transient current exists for nonactin- $\mathrm{K}^{+}$in the absence of $3 P I$ and in the presence of $55 \mu \mathrm{M} 3 \mathrm{PI}$, but the results in these cases are more ambiguous. In the absence of 3PI a voltage-dependent $\tau$ ranging from $11 \mu \mathrm{sec}$ at $25 \mathrm{mV}$ to $80 \mu \mathrm{sec}$ at $200 \mathrm{mV}$ was observed but this is probably an artifact of the response time of the amplifier since the noise/signal ratio was large, making the use of large feedback resistance and capacitance necessary. When $55 \mu \mathrm{M} 3 \mathrm{PI}$ was present two relaxations were observed. The transient with a short time constant ( $10 \mu \mathrm{sec})$ was probably due to bilayer capacitive charging and thus it was not analyzed. The other transient was voltage independent with $\tau=16-27 \mu \mathrm{sec}$. The current level in this case was sufficiently large that only moderate feedback resistance and capacitance values were used. Since the amplifier feedback values were kept nearly the same at all voltages, the voltage-independent $\tau$ can be attributed to amplifier response rather than to any ion transport process. Earlier steady state measurements gave a value of 0.18 for parameter $A$ when $55 \mu M$ 3PI was present in the aqueous solution (see Table IV), which implies that nonactin- $\mathrm{K}^{+}$transport is being kinetically limited at that $3 \mathrm{PI}$ concentration. We conclude that if any transient current existed in 


\section{TABLE V}

RELAXATION AMPLITUDE AND TIME CONSTANT OF PHOSPHATIDYLCHOLINE/ CHOLESTEROL BILAYER IN THE PRESENCE OF NONACTIN, $0.1 \mathrm{M} \mathrm{KCl}$ and $80 \mu \mathrm{M}$ 3-PHENYLINDOLE

\begin{tabular}{ccc}
\hline $\begin{array}{c}\text { Voltage } \\
(\mathrm{mV})\end{array}$ & $\alpha$ & $\begin{array}{c}\tau \\
(\mu \mathrm{sec})\end{array}$ \\
\hline & & 4.4 \\
50 & 8.6 & 3.7 \\
75 & 17.5 & 4.9 \\
100 & 3.1 & 4.3 \\
125 & 11.4 & 4.6 \\
150 & 3.3 & 5.6 \\
175 & 1.8 & 4.9 \\
\hline
\end{tabular}

these conditions it had a small amplitude and decayed with a time constant less than $30 \mu \mathrm{sec}$.

In a thorough review article on the carrier mechanism, Hladky (1979) has pointed out inconsistencies which arise when the results of steady state and transient current measurements are combined in order to obtain values for all the rate constants. He speculates that either the assumptions leading to Eq. (4) in Chapter $V$ are too simplistic or that the transport scheme of Fig. 19 needs major modifications. To demonstrate the former hypothesis, he gives examples in which slightly different assumptions give entirely different results for the relaxation amplitudes. In particular, the form of the voltage dependence of the rate constants is very much open to question. Hladky (1979) also proposes a more complex transport scheme which may reflect the true physical situation better and eventualiy lead to agreement between steady state and transient techniques. The possibility that 
the absence of the transient predicted at high [3PI] was related not to instrument resolution but to some subtle deficiency in the carrier theory should, therefore, not be discounted. 


\title{
CHAPTER VII
}

\author{
CONCLUSION
}

\section{SUMMARY OF EXPERIMENTAL RESULTS}

In this study the physical effects of 3-phenylindole (3PI) were investigated using well-defined model membrane systems containing no metabolic pathways. We found that at concentrations of $5 \mu \mathrm{M}$ to $80 \mu \mathrm{M}$ 3PI, the pesticide adsorbed strongly to lipids, producing changes in electrical potential of the aqueous/lipid interface, changes in bilayer fluidity, and changes in electrical conductivity induced by both carriers and lipophilic ions.

The adsorption of 3PI to egg phosphatidylcholine/cholesterol monolayers was well described by a Langmuir adsorption isotherm, from which it was determined that the adsorption coefficient of 3PI was $1.35 \times 10^{-4} \mathrm{~m}$ and the maximum adsorbed surface density of 3PI was $1.11 \times 10^{-6}$ moles $/ \mathrm{m}^{2}$. The surface potential of the lipid monolayer was decreased by as much as $200 \mathrm{mV}$.

We detected no changes in the electrical conductivity of egg phosphatidylcholine/cholesterol bilayers in the presence of 3PI, and therefore conclude that 3PI does not act as a carrier of ions or as a pore through which ions could permeate the bilayer. We found that 3PI exerted an indirect effect on electrical conductivity of bilayers as evidenced by the following observations. 3PI increased the conductivity induced by $\mathrm{TPhA}^{+}$and nonactin- $\mathrm{K}^{+}$by several orders of magnitude 
and decreased the conductivity induced by $\mathrm{TPhB}^{-}$and $\mathrm{DPA}^{-}$by a factor of less than ten. We have shown that these changes in conductivity are consistent with a decrease in electrical potential of the bilayer interior caused by the presence of 3PI. The disparity between the magnitude of enhancement of cation conductivity and inhibition of anion conductivity was shown to be compatible with decreased viscosity or decreased bilayer thickness brought about by the presence of 3PI.

By using two dissimilar lipophilic anions, we determined that the small magnitude of the change in lipophilic anion conductivity was not related to the detailed chemical structure of the anion, and that fluidity or thickness changes contributed to $\mathrm{TPhB}^{-}$transport to a greater extent than to $\mathrm{DPA}^{-}$transport. In the same experiment we found that $3 \mathrm{PI}$ decreased the partition coefficients of $\mathrm{TPhB}^{-}$and $\mathrm{DPA}^{-}$ by less than a factor of three.

A7though 3PI caused a slightly greater enhancement of nonactin- $\mathrm{K}^{+}$ zero voltage conductivity than of $\mathrm{TPhA}^{+}$zero voltage conductivity, we believe this is insufficient evidence to decide whether 3PI affects any of the steps specific to the carrier mechanism. On the other hand, we found in voltage-dependence studies of normalized conductivity that 3PI did not kinetically limit $\mathrm{TPhA}^{+}$transport but that at concentrations greater than $25 \mu \mathrm{M}, 3 \mathrm{PI}$ caused a kinetic limitation to nonactin$\mathrm{K}^{+}$transport. We were unable to detect any transient currents in the kinetically limited regime for nonactin- $\mathrm{K}^{+}$. 


\section{POSSIBLE BIOLOGICAL SIGNIFICANCE OF THE}

EXPERIMENTAL RESULTS

Relating our experiments using model membranes to the biological changes which 3PI induces requires speculation about how physical changes in the lipid portion of the membrane influence not only the lipids but also the transport proteins and the metabolic pathways present in the organism but absent in the model membranes. First of a11, note that the physical changes we found were all observable within minutes after the model membranes came in contact with 3PI. The uptake of 3PI is probably slower in fungal mycelium than in lipid monolayers or bilayers due to the presence of the cell wall in the fungus. Our finding that $3 P I$ did not act as a pore in $\mathrm{PC} / \mathrm{cholesterol}$ bilayers is consistent with the fact that $3 P I$ has a fungistatic rather than a fungicidal action; that is, the damage 3PI causes is not so great that it is irreversible, whereas the presence of pores is usually associated with gross permeability changes causing irreversible damage.

The fact that degradation and biosynthesis of phospholipids play a central role in the fungistatic action of $3 P I$ is apparent from the evidence given by Hoppe, Kerkenaar, and Sijpesteijn (1976a) [HKSa] that the fungistatic effect occurs two to three days after treatment with 3PI and is reversible by addition of phospholipids. These changes in biosynthetic or degradative pathways are presumably responsible for the changes in phospholipid composition and free fatty acid concentration as described in Chapter I. HKSa found that 3PI had no effect on $\mathrm{O}_{2}$ uptake which indicates that respiration pathways necessary for phospholipid synthesis and for ion transport are intact. In further 
investigations Hoppe, Kerkenaar, and Sijpesteijn (1976b) [HKSb] found that $3 P I$ inhibited ${ }^{32} P_{i}$ uptake within a period of one to several hours after treatment with the pesticide. This change in transport is not primarily responsible for growth inhibition, but a deficiency of phosphorus could decrease the rate of phospholipid biosynthesis and hence be related to subsequent changes in phospholipid concentration and eventual growth inhibition. We found that 3PI, at concentrations which HKSb found to decrease $P_{i}$ uptake, influenced ion transport in lipid bilayers by changing the dipole potential at the lipid/aqueous interface and by changing the fluidity of the bilayer interior. Assuming that these same physical effects occur to some extent when 3PI adsorbs to fungal membranes, we speculate that these effects could be responsible for decreasing phosphate transport in the fungus. We are not aware of any investigations on phosphate transport in Aspergillus niger, but it seems probable that phosphate transport in this species occurs both by mediated transport and by an energydependent carrier, as discussed in Chapter I for Neurospora crassa, yeast, and mitochondria. In general we postulate that when $3 P I$ adsorbs to the membrane and changes the local electrostatic potential, the protein(s) responsible for phosphate transport change conformation due to electrostatic interactions. This type of electrostatic control of enzyme activity has been reported for $\mathrm{Na}^{+}+\mathrm{K}^{+}$-ATPase preparation containing charged lipids (Ahrens, 1981). If the conformational change makes binding to $P_{i}$ less energetically favorable, then $P_{i}$ transport would be inhibited. Alternatively, the increased fluidity of the membrane interior due to the presence of 3PI may infiuence the location 
or diffusion rate of the protein(s) involved in the transport process. It is not clear at this time how a specificity to the $P_{j}$ transport system would occur.

In view of possible influences on phospholipid degradation pathways, the effect of $3 P I$ on the concentration of free fatty acids and on phospholipase activity should be considered. HKSa report that the concentration of free fatty acids in $\underline{A}$. niger increased dramatically two days after treatment with 3PI, a phenomenon which could be due either to a disruption of normal phospholipid biosynthesis or to increased degradation of phospholipids. Since free fatty acids are produced when phospholipase acts on phospholipids, it would be valuable to quantitate the effect of 3PI on phospholipase activity in the fungal membrane. Possibly the increased membrane fluidity brought about by the presence of 3PI allows for better contact between the phospholipase molecule and that portion of the phospholipid molecule in which the change occurs, presumably near the glycerol backbone. In addition to fatty acids, another product of phosphatidylcholine degradation is Tysolecithin, for which HKSa did not assay. If phospholipid degradation is accelerated by $3 P I$, then increased quantities of Tysolecithin would be expected. It is known that an abnormally high concentration of lysolecithin, a molecule having a wedge shape preventing it from fitting in well with a normal bilayer of dual-chained lipids, causes disruption of liposomes composed only of lipid (Bangham and Horne, 1964), and causes leakage of the intracellular contents of red blood cells (Reman et al., 1969). Thus the presence of elevated lysolecithin concentrations could account for the slight leakage of ${ }^{32} \mathrm{p}$ - 
labeled compounds from fungal mycelia treated with $3 P I$ as reported by HKSa.

\section{SUGGESTIONS FOR FUTURE RESEARCH}

In this study of the physical effects of 3PI, we found indications that 3PI decreased the viscosity of lipid bilayers. There are several types of experiments (e.g., differential scanning calorimetry, nuclear magnetic resonance, electron spin resonance, and fluorescence spectroscopy) which are traditionally used to detect fluidity changes, and it would be beneficial to use one or more of these methods to measure the effect of 3PI on viscosity in a more direct manner. We found that 3PI fluoresces brightly when illuminated with ultraviolet light of wavelength $2537 \AA$, and this phenomenon could perhaps be utilized in viscosity measurements, thereby eliminating the need for a foreign probe molecule.

Dekker, Selling, and Overeem (1975) have tested 64 substituted indoles for antifungal activity and attempted to relate the biological activity with lipophilicity of the analogs and location of the substituent group with respect to the $\mathrm{NH}$ group. They found that 2-phenylindole has almost no fungicidal activity whereas the nearly identical compound 3PI was one of the most toxic analogs out of all those tested. An examination of the effect of 2-phenylindole on the electrical conductivity of lipid bilayers and on lipid monolayer surface potential would aid in testing the correlation between the physical and biological effects as discussed in the preceding section. 
The influence of $3 P I$ on $P_{i}$ transport in $\underline{A}$. niger could be open to in vitro investigation if the techniques used to isolate the $P_{i}$ transport protein in mitochondria (Wohlrab, 1980) can be used as a basis to isolate the $P_{i}$ transport protein in $\underline{A}$. niger. Changes in $P_{i}$ transport in a reconstituted system as a function of 3PI concentration, lipid composition, and temperature would undoubtedly yield much information.

Since it is still an open question whether 3PI affects degradation of phospholipids, it would seem prudent to check for the buildup of lysophospholipids in fungal mycelia treated with 3PI. The role of phospholipase could perhaps be further investigated by in vitro tests of the effect of 3PI on lipase activity, e.g., by an extension of the study of Colacicco (1969) on the interaction between phospho1 ipase $A$ and monolayers of various lipoproteins.

Since HKSa conclude that interaction of 3PI with phospholipids is a crucial part of the fungistatic action of 3PI, it would be helpful to do monolayer adsorption experiments as described in Chapter II using monolayers of non-phospholipids to determine whether 3PI adsorbs preferentially to phospholipids. In a similar vein, the conductivity studies could be repeated with lipids other than phospholipids to determine whether specific 3PI-1ipid interaction is involved in conductivity changes.

In connection with our studies of 3PI, we attempted to determine the effect of $3 P I$ on the proton nuclear magnetic resonance (NMR) spectra of PC/cholesterol liposomes and from these preliminary studies it became obvious that several experimental difficulties would have 
to be overcome before quantitative results could be obtained. The main difficulty was the requirement for high concentrations of lipid necessary to generate the NMR signal. Whereas in the conductivity experiments a small area of bilayer is bathed by a large volume of aqueous solution containing 3PI, in NMR studies a large amount of 1 ipid in the form of spherical liposomes is bathed by a small volume ( $2 \mathrm{ml})$ of aqueous solution containing 3PI. Thus, due to the large partition coefficient of 3PI, the liposomes can actually deplete the solution of its 3PI content. HKSb found that 3PI, when mixed with lecithin prior to addition of water for liposome formation, prevented the dispersion of phospholipids in salt solution. The second difficulty encountered in using liposomes for NMR was the reproducibility of the degree of sonication; the sonicator used in these preliminary studies did not have automatic intensity control so the intensity of ultrasonic energy tended to vary with time. Unsonicated lipid dispersions produce very broad peaks in the NMR spectrum and well-sonicated liposomes produce relatively sharp peaks. Therefore, depending on the degree of sonication, apparently different spectra were obtained.

In the present study the analysis of the data for adsorption of 3PI onto lipid monolayers was based only on geometrical arguments. An important complement to these experimental investigations would be the development of a general theory of adsorption of lipophilic pesticides onto lipid monolayers, beginning with the condition of equilibrium of the pesticide between aqueous phase and monolayer, and taking into account the variable area of contact between aqueous phase and monolayer. The approximation used for the area of the pesticide 
molecule could perhaps be improved on by a more detailed consideration of its freedom to rotate and to interact with surrounding molecules. 


\section{REFERENCES}

Ahmad, P., Mellors, A. 1978. Nuclear magnetic resonance studies on 1iposomes: Effects of steroids on lecithin fatty acyl chain mobility. ‥ Memb. Biol. $41: 235$

Ahrens, M.-L. 1981. Electrostatic control by lipids upon the membranebound $\left(\mathrm{Na}^{+}+\mathrm{K}^{+}\right)$-ATPase. Biochim. Biophys. Acta $642: 252$

Albrecht, O., Gruler, H. , Sackman, E. 1978. Polymorphism of phospholipid monolayers. Le Journal de Physique 39:301

Andersen, 0.S., Feldberg, S., Nakadomari, H., Levy, S., McLaughi,in, S. 1978. Electrostatic interactions among hydrophobic ions in lipid bi layer membranes. Biophys. ‥ 21:35

Andersen, 0.S., Finkelstein, A., Katz, I., Cass, A. 1976. Effect of phioretin on the permeability of thin lipid membranes. J. Gen. Physiol. 67:749

Andersen, 0.S., Fuchs, M. 1975. Potential energy barriers to ion transport within lipid bilayers. Biophys. J. 15:795

Anderson, E. 1971. Modern Physics and Quantum Mechanics. Saunders, Philadelphia

Andreoli, T.E., Monahan, M. 1968. The interaction of polyene antibiotics with thin lipid membranes. ‥ Gen. Physiol. 52:300

Armstrong, W.McD. 1972. Ion transport and related phenomena in yeast and other micro-organisms. In: Transport and Accumulation in Biological Systems by E.J. Harris. p. 407. Butterworth \& Co., London

Bangham, A.D., Horne, R.W. 1964. Negative staining of phospholipids and their structural modification by surface-active agents as observed in the electron microscope. J. Mol. Biol. 8:660

Barratt, M.D., Weaver, A.C. 1979. The interaction of 3,3',4',5-tetrachlorosalicylanilide with phosphatidylcholine bilayers. Biochim. Biophys. Acta 555:337

Benz, R., Cros, D. 1978. Influence of sterols on ion transport through lipid bilayer membranes. Biochim. Biophys. Acta 506:265 
Benz, R., Fröhlich, 0., Läuger, P. 1977. Influence of membrane structure on the kinetics of carrier mediated ion transport through lipid bilayers. Biochim. Biophys. Acta 464:465

Benz, R., Gisin, B.F. 1978. Influence of membrane structure on ion transport through lipid bilayer membranes. J. Memb. Biol. 40:293

Benz, R., Läuger, P. 1977. Transport kinetics of dipicrylamine through lipid bilayer membranes. Effects of membrane structure. Biochim. Biophys. Acta 468:245

Benz, R., Läuger, P., Janko, K. 1976. Transport kinetics of hydrophobic ions in lipid bilayer membranes. Charge-pulse relaxation studies. Biochim. Biophys. Acta 455:701

Benz, R., Stark, G. 1975. Kinetics of macrotetrolide-induced ion transport across lipid bilayer membranes. Biochim. Biophys. Acta $382: 27$

Benz, R., Stark, G., Janko, K., Läuger, P. 1973. Val inomycin-mediated ion transport through neutral 1ipid membranes: Influence of hydrocarbon chain length and temperature. ‥ Memb. Biol. 14:339

Berden, J.A., Barker, R.W., Radda, G.K. 1975. NMR studies on phospholipid bilayers: Some factors affecting lipid distribution. Biochim. Biophys. Acta 375:186

Bielawski, J., Thompson, T.E., Lehninger, A.L. 1966. The effect of 2,4-dinitrophenol on the electrical resistance of phospholipid bilayer membranes. Biochem. Biophys. Res. Commun. 24:948

Bolis, L., Gomperts, B.D. 1972. Red blood cells. In: Transport and Accumulation in Biological Systems by E.J. Harris. p. 93. Butterworth \& Co., London

Burns, D.J.W., Beever, R.E. 1977. Kinetic characterization of the two phosphate uptake systems in the fungus Neurospora crassa. Bacteriol. 132:511

Cadenhead, D.A. 1977. Monomolecular films and membrane structure. In: Structures of Biological Membranes. S. Abrahamson and I. Pascher, editors. p. 63. Plenum Press, New York

Cadenhead, D.A., Kellner, B.M.J., Phillips, M.C. 1976. The miscibility of dipalmitoyl phosphatidyicholine and cholesterol in monolayers. J. Col1. Interface Sci. 57:224

Chapman, D. 1975. Phase transitions and fluidity characteristics of lipids and cell membranes. Quart. Rev. Biophys. 8:185 
Chapman, D., Penkett, S.A. 1966. Nuclear magnetic resonance spectroscopic studies of the interaction of phospholipids with cholestero1. Nature 211:1304

Ciani, S., Eisenman, G., Szabo, G. 1969. A theory for the effects of neutral carriers such as the macrotetralide actin antibiotics on the electric properties of bilayer membranes. $\underline{\text {. }}$ Memb. Biol. $1: 1$

Coetzee, J.F., Sharpe, W.R. 1971. Solute-solvent interactions. VI. Specific interactions of tetraphenylarsonium, tetraphenylphosphonium, and tetraphenylborate ions with water and other solvents. J. Phys. Chem. 75:3141

Colacicco, G. 1969. Applications of monolayer techniques to biological systems: Symptoms of specific lipid-protein interactions. $\underline{\mathrm{j}}$. Coll. Interface Sci. 29:345

Corbett, J.R. 1974. The Biochemical Mode of Action of Pesticides. Academic Press, New York

Costa, M., Priami, L., Bordi, S. 1976. Apparatus for the detection of surface potential. J. Electroanal. Chem. 70:229

Coty, W.A., Wehrle, J.P., Pedersen, P. 1979. Measurement of phosphate transport in mitochondria and in inverted inner membrane vesicles of rat liver. In: Methods in Enzymology, Vol. LVI. S. Fleischer and L. Packer, editors. p. 353. Academic Press, New York

Darke, A., Finer, E.G., Flook, A.G., Phillips, M.C. 1972. Nuclear magnetic resonance study of lecithin-cholesterol interactions. J. Mol. Biol. $63: 265$

Davis, B.D., Dulbecco, R., Eisen, H.N., Ginsberg, H.S., Wood, W.B., Jr. 1973. Microbiology. Harper \& Row, Hagerstown, Maryland

Dekker, W.H., Selling, H.A., Overeem, J.0. 1975. Structure-activity

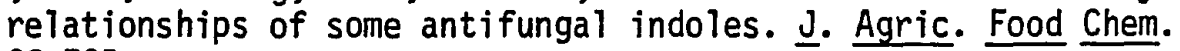
23:785

Delahay, P. 1965. Double Layer and Electrode Kinetics. Interscience Publishers, New York

delevie, R., Rangarajan, S.K., Seelig, P.F., Andersen, 0.S. 1979. On the adsorption of phloretin onto a black lipid membrane. Biophys. J. 25:295

Demel, R.A., Joos, P. 1968. Interaction between lecithins and cholesterol at the air-water and oil-water interfaces. Chem. Phys. Lipids 2:35 
Deme1, R.A., van Deenan, L.L.M., Kinsky, S.C. 1965. Penetration of 1 ipid monolayers by polyene antibiotics. J. Biol. Chem. 240:2749

Dodge, A.D. 1971. The mode of action of the bipyridylium herbicides, paraquat and diquat. Endeavor 30:130

Eytan, G.D., Matheson, M.J., Racker, E. 1976. Incorporation of mitochondrial membrane proteins into liposomes containing acidic phospholipids. J. Biol. Chem. 251:6831

Finkelstein, A. 1970. Weak-acid uncouplers of oxidative phosphorylation. Mechanism of action on thin lipid membranes. Biochim. Biophys. Acta 205:1

Finkelstein, A., Cass, A. 1968. Permeability and electrical properties of thin lipid membranes. J. Gen. Physiol. 52:145s

Fischer, E., Schmidt, T. 1888. Ueber Pr. 3.Phenyl indol. Berichte Deutschen Chemischen Gesellschaft. 21:1811

Gaines, G.L.,Jr. 1966. Insoluble Monolayers at Liquid-Gas Interfaces. Interscience Publishers, New York

Gaines, G.L.,Jr. 1978. The thermodynamic equation of state for insoluble monolayers I. Uncharged films. ‥ Chem. Phys. 69:924

Goddard, E.D., editor. 1975. Monolayers. Advances in Chemistry Series, No. 144. American Chemical Society, Washington, DC

Goldman, D.E. 1943. Electrical properties of membranes. $\underline{J}$. Gen. Physiol. 27:37

Graham, D.E., Phil1ips, M.C. 1979a. Proteins at 1 iquid interfaces I. Kinetics of adsorption and surface denaturation. $\underline{3}$. Coll. Interface Sci. 70:403

Graham, D.E., Phillips, M.C. 1979b. Proteins at liquid interfaces II. Adsorption isotherms. J. Coll. Interface Sci. 70:415

Hanai, T., Haydon, D.A., Taylor, J. 1965. The variation of capacitance and conductance of bimolecular lipid membranes with area. $\underline{\mathrm{J}}$. Theor. Biol. 9:433

Haydon, D.A., Hladky, S.B. 1972. Ion transport across thin lipid membranes: A critical discussion of mechanisms in selected systems. Quart. Rev. Biophys. 5:187

Hilton, B.D., O'Brien, R.D. 1973. The effects of DDT and its analogs upon lecithin and other monolayers. Pest. Biochem. Physiol. 3:206

Hladky, S.B. 1972. The steady-state theory of the carrier transport of ions. ‥ Memb. Biol. 10:67 
Hladky, S.B. 1973. The effect of stirring on the flux of carriers into black lipid membranes. Biochim. Biophys. Acta 307:261

Hladky, S.B. 1974. The energy barriers to ion transport by nonactin across thin lipid membranes. Biochim. Biophys. Acta 352:71

Hladky, S.B. 1975. Tests of the carrier model for ion transport by nonactin and trinactin. Biochim. Biophys. Acta 375:327

H1adky, S.B. 1979. The carrier mechanism. In: Current Topics in Membranes and Transport. F. Bronner and A. Kleinzeller, editors. p. 53. Academic Press, New York

H7adky, S.B., Haydon, D.A. 1970. Discreteness of conductance change in bimolecular lipid membranes in the presence of certain antibiotics. Nature 225:451

Hoppe, H.H., Kerkenaar, A., Sijpesteijn, A.K. 1976a. On the mode of action of 3-phenyl indole towards Aspergillus niger. Pest. Biochem. Physiol. 6:413

Hoppe, H.H., Kerkenaar, A., Sijpesteijn, A.K. 1976b. Interaction with phospholipids as a possible mode of action of 3-phenylindole on Aspergillus niger. Pest. Biochem. Physiol. 6:422

Ikawa, M. 1967. Bacterial phosphatides and natural relationships. Bacteriol. Rev. 31:54

Israelachvili, J.N., Ninham, B.K. 1977. Intermolecular forces--the long and short of it. ․ Coll. Interface Sci. 58:14

Johnson, J.H., Grula, E.A., Staerke1, R., Fung, B.M., Mangum, B.L. 1980. Modification of cell membrane lipids in Micrococcus lysodeikticus induced by pantoyl lactone. ‥ Memb. Biol. 56:49

Kagawa, Y., Kandrach, A., Racker, E. 1973. Partial resolution of the enzymes catalyzing oxidative phosphorylation XXVI. Specificity of phospholipids required for energy transfer reactions. $\underline{J}$. Biol. Chem. 248:676

Kellner, B.M.J., Müller-Landau, F., Cadenhead, D.A. 1978. The temperature-dependence characterization of insoluble films at the airwater interface. ‥ Col1. Interface Sci. 66:597

Ketterer, B., Neumcke, B., Läuger, P. 1971. Transport mechan ism of hydrophobic ions through lipid bilayer membranes. ․ Memb. Biol. $5: 225$

Kinsky, S. 1961. Alterations in the permeability of Neurospora Crassa due to polyene antibiotics. J. Bacteriol. $82: 8 \overline{89}$ 
Kinsky, S. 1962. Nystatin binding by protoplasts and a particulate fraction of Neurospora Crassa, and a basis for the selective toxicity of polyene antifungal antibiotics. Proc. Nat. Acad. Sci. USA 48:1049

Knowles, A.F., Racker, E. 1975. Formation of adenosine triphosphate from $\mathrm{P}_{i}$ and adenosine diphosphate by purified $\mathrm{Ca}^{2+}$-adenosine triphosphatase. J. Biol. Chem. 250:1949

Kornberg, R.D., McConnel1, H.M. 1971a. Inside-outside transitions of phospholipids in vesicle membranes. Biochemistry 10:1111

Kornberg, R.D., McConnell, H.M. 1971b. Lateral diffusion of phospholipids in a vesicle membrane. Proc. Nat. Acad. Sci. USA 68:2564

Ladbrooke, B.D., Williams, R.M., Chapman, D. 1968. Studies on lecithincholesterol-water interactions by differential scanning calorimetry and X-ray diffraction. Biochim. Biophys. Acta 150:333

Lampen, J.0., Arnow, P.M., Safferman, R.S. 1960. Mechanism of protection by sterols against polyene antibiotics. J. Bacteriol. 80:200

Langmuir, 1. 1917. The constitution and fundamental properties of solids and Tiquids. II. Liquids. J. Am. Chem. Soc. 39:1848

Läuger, P. 1973. Ion transport through pores: A rate-theory analysis. Biochim. Biophys. Acta 311:423

Läuger, P. 1980. Kinetic properties of ion carriers and channels. J. Memb. Biol. 57:163

Lea, E.J.A., Croghan, P.C. 1969. The effect of 2,4-dinitrophenot on the properties of thin phospholipid films. ‥ Memb. Biol. 1:225

LeBlanc, 0.H.,Jr. 1969. Tetraphenylborate conductance through lipid bilayer membranes. Biochim. Biophys. Acta 193:350

Lee, A.G. 1975. Functional properties of biological membranes: A physicochemical approach. Progress Biophys. Mol. Biol. 29:3

Liberman, E.A., Topaly, V.P. 1968. Selective transport of ions through bimolecular phospholipid membranes. Bjochim. Biophys. Acta $163: 125$

Liberman, Ye.A., Topaly, V.P. 1969. Permeability of bimolecular phospholipid membranes for fat-soluble ions. Biophysics 14:477

Lowendorf, H.S., Bazinet, G.F., Jr., Slayman, C.W. 1975. Phosphate transport in Neurospora. Derepression of a high-affinity transport system during phosphorus starvation. Biochim. Biophys. Acta 389:541 
Lowendorf, H.S., Slayman, C.L., Slayman, C.W. 1974. Phosphate transport in Neurospora. Kinetic characterization of a constitutive, lowaffinity transport system. Biochim. Biophys. Acta 373:369

Marini, F., Arnow, P., Lampen, J.0. 1961. The effect of monovalent cations on the inhibition of yeast metabolism by nystatin. J. Gen. Microbiol. 24:51

Markin, V.S., Krishtalik, L.I., Liberman, Ye.A., Topaly, V.P. 1969. Mechanism of conductivity of artificial phospholipid membranes in presence of ion carriers. Biofizika 14:256

McClellan, A.L. 1974. Tables of Experimental Dipole Moments, Vol. 2. Rahara Enterprises.

McGivan, J.D., Klingenberg, M. 1971. Correlation between $\mathrm{H}^{+}$and anion movement in mitochondria and the key role of the phosphate carrier. Eur. J. Biochem. 20:392

McLaught in, S.G.A., Dilger, J.P. 1980. Transport of protons across membranes by weak acids. Physiol. Rev. 60:825

Merkle, M.G., Leinweber, C.L., Bovey, R.W. 1965. The influence of light, oxygen and temperature on the herbicidal properties of paraquat. Plant Physiol. 40:832

Mueller, P., Rudin, D.0., Tien, H., Wescott, W.C. 1963. Methods for the formation of single bimolecular lipid membrane's in aqueous

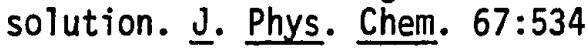

Muramatsu, M. 1973. Radioactive tracers in surface and colloid science. In: Surface and Colloid Science, Vol. 6. E. Matijevic, editor. p. 101. Wiley and Sons, New York

Nagle, J.F., Scott, H.L. 1978. Biomembrane phase transitions. Physics Today $31: 38$

Nelson, P.V., Reid, R. 1971. Selectivity mechanism for the differential destruction of plant tissues by methyl decanoate emulsion. Amer. J. Botany 58:249

Nester, E.W., Roberts, C.E., Pearsa11, N.N., McCarthy, B.J. 1978. Microbiology. Holt, Rinehart and Winston, New York

Newton, B.A. 1956. The properties and mode of action of the polymyxins. Bacteriol. Rev. 20:14

O'Leary, W.M. 1967. The Chemistry and Metabolism of Microbial Lipids. World Publishing Company, New York 
Paltauf, F., Hauser, H., Phillips, M.C. 1971. Monolayer characteristics of some 1,2-diacyl, 1-alkyl-2-acyl and 1,2-dialkyl phospholipids at the air-water interface. Biochim. Biophys. Acta 249:539

Papahadjopoulos, D. 1968. Surface properties of acidic phosphol ipids: Interaction of monolayers and hydrated liquid crystals with uniand bi-valent metal ions. Biochim. Biophys. Acta 163:240

Papahadjopoulos, D., Cowden, M., Kimelberg, H. 1973. Role of cholesterol in membranes: Effects on phospholipid-protein interactions, membrane permeability and enzymatic activity. Biochim. Biophys. Acta $330: 8$

Phillips, M.C., Chapman, D. 1968. Monolayer characteristics of saturated 1,2-diacyl phosphatidylcholines (lecithins) and phosphatidylethanolamines at the air-water interface. Biochim. Biophys. Acta 163:301

Phillips, M.C., Finer, E.G., Hauser, H. 1972. Differences between conformations of lecithin and phosphatidylethanolamine polar groups and their effects on interactions of phospholipid bilayer membranes. Biochim. Biophys. Acta 290:397

Pickar, A.D., Benz, R. 1978. Transport of oppositely charged 1ipophilic probe ions in lipid bilayer membranes having various

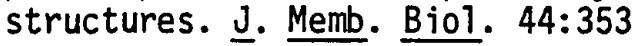

Racker, E. 1979. Reconstitution of membrane processes. In: Methods in Enzymology, Vol. LV, Part F. S. Fleischer and L. Packer, editors. p. 699. Academic Press, New York

Ragan, C.I., Racker, E. 1973. Partial resolution of the enzymes catalyzing oxidative phosphorylation. XXVIII: The reconstitution of the first site of energy conservation. J. Biol. Chem. 248:2563

Reman, F.C., Deme1, R.A., DeGrier, J., vanDeenen, L.L.M., Eibl, H., Westphal, 0. 1969. Studies on the lysis of red cells and bimolecular lipid leaflets by synthetic lysolecithins, lecithins and structural analogs. Chem. Phys. Lipids 3:221

Rich, G.T. 1973. The interaction of 2,4-dinitrophenol with phospholipids at phospholipid-water interfaces. Chem. Phys. Lipids $10: 253$

Roels, 0.A., Shah, D.0. 1969. Molecular interactions in lecithinretinol monolayers. ‥ Coll. Interface Sci. 29:279

Saier, M., Stiles, C. 1975. Molecular Dynamics in Biological Membranes. Springer-Verlag, New York

Shah, D. 1970. Surface chemistry of 1ipids. Advances in Lipid Research $8: 347$ 
Shah, D., Schulman, J.H. 1965. Binding of metal ions to monolayers of lecithins, plasmalogen, cardiolipin, and dicetyl phosphate. J. Lipid Res. 6:341

Shah, D., Schulman, J.H. 1967. Influence of calcium, cholesterol, and unsaturation on lecithin monolayers. ‥ Lipid Res. 8:215

Shinitzky, M., Dianoux, A.-C., Gitler, C., Weber, G. 1971. Microviscosity and order in the hydrocarbon region of micelles and membranes determined with fluorescent probes. I. Synthetic micelles. Biochemistry 10:2106

Simoni, R.D. 1972. Macromolecular characterization of bacterial transport systems. In: Membrane Molecular Biology. C.F. Fox and A.D. Keith, editors. p. 289. Sinauer Associates, Inc., Stamford, Conn.

Singer, S.J., Nicolson, G.L. 1972. The fluid mosaic model of the structure of cell membranes. Science 175:720

Smejtek, P., Hsu, K., Perman, W.H. 1976. Electrical conductivity in 1ipid bilayer membranes induced by pentachlorophenol. Biophys. J. $16: 319$

Smejtek, P., Paulis-Illangasekare, M. 1979a. Modification of ion transport in lipid bilayer membranes in the presence of 2,4-dichlorophenoxyacetic acid $(2,4-D)$. I. Enhancement of cationic conductance and changes of the kinetics of nonactin-mediated transport of potassium. Biophys. J. 26:441

Smejtek, P., Paulis-I1langasekare, M. 1979b. Modification of ion transport in lipid bilayer membranes in the presence of 2,4-dichlorophenoxyacetic acid $(2,4-D)$. II. Suppression of tetraphenylborate conductance and changes of interfacial potentials. Biophys. J. $26: 467$

Smith, T. 1967. Monolayers on water. I. A theoretical equation for the liquid expanded state. $\mathrm{J}$. Coll. Interface Scj. 23:27

Standish, M.M., Pethica, B.A. 1967. Interactions in phospholipidcholesterol mixed monolayers at the air/water interface. Biochim. Biophys. Acta 144:659

Stark, G., Benz, R. 1971. The transport of potassium through lipid bilayer membranes by the neutral carriers valinomycin and monactin. J. Memb. Biol. 5:133

Stark, G., Benz, R., Pohl, G.W., Janko, K. 1972. Valinomycin as a probe for the study of structural changes of black lipid membranes. Biochim. Biophys. Acta 266:603 
Stark, G., Ketterer, B., Benz, R., Läuger, P. 1971. The rate constants of valinomycin-mediated ion transport through thin lipid membranes. Biophys. ‥ 11:981

Szabo, G. 1974. Dual mechanism for the action of cholesterol on membrane permeability. Nature 252:47

Szabo, G. 1976. The influence of dipole potentials on the magritude and the kinetics of ion transport in lipid bilayer membranes. In: Extreme Environments: Mechanism of Microbial Adaptation. M.R. Heinrich, editor. p. 321. Academic Press, New York

Szabo, G., Eisenman, G., McLaugh1 in, S.G.A., Krasne, S. 1972. Ionic probes of membrane structures. Annals N. ‥ Acad. Sci. 195:273

Tinoco, J., McIntosh, D.J. 1970. Interactions between cholesterol and lecithins in monolayers at the air-water interface. Chem. Phys. Lipids $4: 72$

Trauble, H., Sackman, E. 1972. Studies of the crystalline-liquid crystalline phase transition of lipid model membranes. III. Structure of a steroid-lecithin system below and above the lipid phase transition. $\mathrm{J}$. Am. Chem. Soc. 94:4499

Triggle, D.J. 1970. Some aspects of the role of lipids in 1ipid-protein interactions and cell membrane structure and function. In: Recent Progress in Surface Science, Vol. 3. J.F. Daniel1i, A.C. Riddiford, and M.D. Rosenberg, editors. p. 273. Academic Press, New York

Tyler, D.D. 1969. Evidence of a phosphate-transporter system in the inner membrane of isolated mitochondria. Biochem. J. 111:665

Van Deenen, L.L.M., Houtsmuller, V.M.T., deHaas, G.H., Mulder, E. 1962. Monomolecular layers of synthetic phosphatides. $\underline{J}$. Pharmacy and Pharmacology 14:429

Wang, C.-C., Bruner, L.J. 1978. Dielectric saturation of the aqueous boundary layers adjacent to charged bilayer membranes. ‥ Memb. Biol. 38:311

Wiegel, F.W., Kox, A.J. 1980. Theories of lipid monolayers. In: Advances in Chemical Physics. I. Prigogine and S.A. Rice, editors. p. 195. John Wiley \& Sons, New York

Wohlrab, H. 1980. Purification and reconstitution of the mitochondrial phosphate transporter. Annais N. Y. Acad. Sci. 358:364

Zähner, H., Maas, W.K. 1972. Biology of Antibiotics. Springer-Verlag, New York 